\title{
ON THE STRICTNESS OF THE QUANTIFIER STRUCTURE HIERARCHY IN FIRST-ORDER LOGIC*
}

\author{
YUGUO HE
}

School of Computer Science, Beijing Institute of Technology, Beijing 100081, China.

e-mail address: hugo274@gmail.com

\begin{abstract}
We study a natural hierarchy in first-order logic, namely the quantifier structure hierarchy, which gives a systematic classification of first-order formulas based on structural quantifier resource. We define a variant of Ehrenfeucht-Fraïssé games that characterizes quantifier classes and use it to prove that this hierarchy is strict over finite structures, using strategy compositions. Moreover, we prove that this hierarchy is strict even over ordered finite structures, which is interesting in the context of descriptive complexity.
\end{abstract}

\section{INTRODUCTION}

One of the major interests of finite model theory is to separate the expressive power of different logics or fragments of logics. Quantifiers are an important logical resource for measuring the logical complexity of problems. The study of fragments of first-order logic (FO) based on quantifier structures, especially quantifier prefixes, has a long history in model theory [2]. However, so far there are few results about the expressive power of such fragments. Walkoe [10 proved that there exists a sentence with prefix $p$ which is different from any sentence with prefix $q$ if $p$ and $q$ are different but with the same length. In the proof, the structures are assumed to be infinite. Afterwards Keisler and Walkoe [3] improved this result by showing its validity over finite structures. Chandra and Harel [1] proved that $\Sigma_{k} \subsetneq \Sigma_{k+1}$ over finite digraphs. Sipser [9] proved a similar result in the context of unbounded fan-in bounded depth circuits.

In 1996, Grädel and McColm [2] established a strict hierarchy based on quantifier classes in the infinitary logic over finite structures and resolved a conjecture of Immerman, i.e. $\Sigma_{i}^{T C} \subsetneq \Sigma_{i+1}^{T C}$ for each $i$. At the same time, they proposed a conjecture on the expressive power of the fragments of FO based on prefixes, which generalized the previous results [10], [3] and [1. In 1998, Rosen [6] confirmed this conjecture and called the strict hierarchy based on these fragments of FO the first-order prefix hierarchy. Actually, Rosen proved a stronger result, which states that, over a single binary relation, for any prefix $p$ there is a first-order

2012 ACM CCS: [Theory of computation]: Logic-Finite Model Theory; [Mathematics of computing]: Discrete mathematics - Graph theory - Trees.

Key words and phrases: Finite Model Theory, Quantifier Structure, Quantifier Class, Ehrenfeucht-Fraïssé Games, Strategy Composition, Point-expansion, Ordered Structures.

* This paper is from a part of the author's PhD thesis. It is an extension and revision of a conference paper published in Proceedings of the 25th Annual IEEE Symposium on Logic in Computer Science. 
sentence $\varphi_{p}$ in prenex normal form with prefix $p$, such that for any sentences $\psi$ in infinitary logic, $\varphi_{p}$ is not equivalent to $\psi$ if $p$ is not embeddable in the "quantifier structure" of $\psi 1^{1}$ However, a stronger version of the conjecture remains open, i.e. whether it holds over finite structures or not [5, 6]. One way to prove the conjecture is to prove a finite version of Rosen's main theorem.

In this paper, we continue this line of study. We define a variant of Ehrenfeucht-Fraïssé games that characterizes quantifier classes and prove the following main result:

Let $S_{1}$ and $S_{2}$ be two finite $\Gamma$-labeled forests. Over the class of all finite digraphs,

$$
\text { if } S_{1} \npreceq e S_{2} \text {, then } \mathrm{FO}\left\{S_{1}\right\} \nsubseteq \mathrm{FO}\left\{S_{2}\right\} \text {. }
$$

The structures we use in the proof are finite trees, which makes it easy to prove a stronger result: The above main result holds even when the structures have a linear order. Here we introduce the ideas that are used to deal with linear order in a simpler context:

Over the class of all ordered finite digraphs,

$$
\text { if } \mathscr{W}\left(S_{1}\right) \nsubseteq \mathscr{W}\left(S_{2}\right) \text {, then } \mathrm{FO}\left\{S_{1}\right\} \nsubseteq \mathrm{FO}\left\{S_{2}\right\}
$$

\section{Preliminaries}

2.1. General background. Let $\mathbb{N}$ and $\mathbb{N}^{+}$denote the set of natural numbers (non-negative integers) and positive natural numbers respectively.

We assume that the readers have basic knowledge about finite model theory. In the following we briefly introduce some necessary background. The readers can cf. the textbook [4] for more of it.

A relational signature consists of a sequence of relation and constant symbols. In this paper, a signature is relational and finite, whenever mentioned.

Let $\sigma=\left\langle R_{1}, \cdots, R_{m}, c_{1}, \cdots, c_{n}\right\rangle$ be a signature, a $\sigma$-structure $\mathfrak{A}$ consists of a universe $|\mathfrak{A}|$ together with an interpretation of

- each $k$-ary relation symbol $R_{i} \in \sigma$ as a $k$-ary relation on $|\mathfrak{A}|$, denoted by $R_{i}^{\mathfrak{A}}$;

- each constant symbol $c_{i} \in \sigma$ as an element in $|\mathfrak{A}|$.

A structure is called finite if its universe is a finite set.

A $\sigma$-structure $\mathfrak{A}^{\prime}$ is a substructure of $\mathfrak{A}$ if the following hold:

(1) $\left|\mathfrak{A}^{\prime}\right| \subseteq|\mathfrak{A}|$;

(2) For any $k$-ary relation $R \in \sigma \cup\{=\}, R^{\mathfrak{A}^{\prime}}=R^{\mathfrak{A}} \cap\left|\mathfrak{A}^{\prime}\right|^{k}$;

(3) For any constant $c \in \sigma, c^{\mathfrak{A}}=c^{\mathfrak{A}}$.

Let $\sigma^{\prime} \subseteq \sigma$. The $\sigma^{\prime}$-reduct of $\mathfrak{A}$, denoted $\mathfrak{A} \mid \sigma^{\prime}$, is obtained from $\mathfrak{A}$ by leaving all the symbols in $\sigma \backslash \sigma^{\prime}$ uninterpreted.

Let $\mathfrak{A}$ and $\mathfrak{B}$ be wo structures of the same signature. An isomorphism between $\mathfrak{A}$ and $\mathfrak{B}$ is a bijection $h:|\mathfrak{A}| \rightarrow|\mathfrak{B}|$ such that the following hold:

(1) For any $k$-ary relation $R \in \sigma \cup\{=\}$ and $\left(a_{1}, \ldots, a_{k}\right) \in|\mathfrak{A}|^{k}$,

$$
\left(a_{1}, \ldots, a_{k}\right) \in R^{\mathfrak{A}} \text { iff }\left(h\left(a_{1}\right), \ldots, h\left(a_{k}\right)\right) \in R^{\mathfrak{B}}
$$

\footnotetext{
${ }^{1}$ Here, the notion "quantifier structure" is from Grädel and McColm [2], which is different from ours (cf. Definition 2.6.
} 
(2) For any constant $c \in \sigma, h\left(c^{\mathfrak{A}}\right)=c^{\mathfrak{B}}$.

Say that two structures $\mathfrak{A}$ and $\mathfrak{B}$ over the same signature are isomorphic if there is an isomorphism between them, denoted $\mathfrak{A} \cong \mathfrak{B}$.

Let $\bar{a}=\left(a_{1}, \cdots, a_{k}\right) \in|\mathfrak{A}|^{k}, \bar{b}=\left(b_{1}, \cdots, b_{k}\right) \in|\mathfrak{B}|^{k}$. Say that $(\bar{a}, \bar{b})$ defines a partial isomorphism between $\mathfrak{A}$ and $\mathfrak{B}$ if $\bar{a}$ contains the elements that interpret all the constants of $\mathfrak{A}, \bar{b}$ contains the elements that interpret all the constants of $\mathfrak{B}$, and the substructure of $\mathfrak{A}$ that is generated by $\bar{a}$ is isomorphic to the substructure of $\mathfrak{B}$ that is generated by $\bar{b}$. More precisely, the following hold:

(1) for any $m$-ary relation symbol $R \in \sigma \cup\{=\}$ and any sequence $\left(i_{1}, \cdots, i_{m}\right)$ of numbers from $[\mathrm{k}]$,

$$
\left(a_{i_{1}}, \cdots, a_{i_{m}}\right) \in R^{\mathfrak{A}} \text { iff }\left(b_{i_{1}}, \cdots, b_{i_{m}}\right) \in R^{\mathfrak{B}} .
$$

(2) for any constant $c \in \sigma$ and any $i \in[k]$,

$$
a_{i}=c^{\mathfrak{A}} \text { iff } b_{i}=c^{\mathfrak{B}} .
$$

We assume that the readers have basic knowledge about first-order logic, especially what is the meaning of "a formula is true in a structure". Without loss of generality, we assume that all the formulas and sentences are in negation normal form, i.e. all negations can only occur immediately before atoms.

Let $\mathfrak{A}$ be a $\sigma$-structure and $\psi$ be a first-order sentence. We use $\mathfrak{A}=\psi$ to denote that $\psi$ is true in $\mathfrak{A}$, and we call $\mathfrak{A}$ a model for $\psi$. Let $\operatorname{Mod}(\psi)$ be the set of models of $\psi$. A property $Q$ over $\sigma$ is a set of $\sigma$-structures closed under isomorphism. Say that $Q$ is expressible, or definable, in FO if there is a sentence $\varphi$ in $\mathrm{FO}$ such that for every $\mathfrak{A}, \mathfrak{A} \in \operatorname{Mod}(\varphi)$ iff $\mathfrak{A} \in Q$.

A linear order is a binary relation that is transitive, antisymmetric and total. Let $\tau$ be a signature. And let $\tau^{\mathrm{ORD}}:=\tau \cup\{\leq\}$ where $\leq$ is interpreted in a $\tau^{\mathrm{ORD}}$-structure as a linear order of its universe.

2.2. $\Gamma$-labeled forests. Let $n \in \mathbb{N}^{+}$. Given a graph $\mathcal{G}=(V, E)$, a directed path $P$ in $\mathcal{G}$ is a sequence of vertices $\left(v_{0}, \cdots, v_{n}\right)$ such that there is an arc from $v_{i}$ to $v_{i+1}$ for any $i<n$. The length of $P$ is $n$. A directed path is nontrivial if the length of the path is nonzero.

Trees are defined in the usual way in computer science. If there is an arrow from a node $a$ to a node $b$, then we call $a$ a father of $b$ and $b$ a child of $a$. In a tree, each node has zero or more children and each node has at most one father. A node which has no father is called a root and a node which has no child is called a leaf. An inner node is any node that has child nodes. A tree is a connected acyclic digraph that has a root and some leaves. A degenerate tree is a directed path.

The height of a tree is the length of a longest directed path in the tree. A forest is composed of disjoint trees. Let $S$ be a forest. Define its height, denoted $h(S)$, as the maximum height of its trees. And define its rank, denoted $r k(S)$, as $h(S)+1$ when $S$ is not empty and 0 otherwise. Let $\Gamma=\{\exists, \forall\}$. A forest is a $\Gamma$-labeled forest if all its nodes are labeled with " $\exists$ " or " $\forall$ ". We call those nodes labeled with " $\exists$ " $\mathscr{E}$ nodes and the other nodes $\mathscr{A}$ nodes.

A $\Gamma$-labeled perfect binary tree is a $\Gamma$-labeled tree where each node, except the leaves, has exactly one $\mathscr{E}$ child and one $\mathscr{A}$ child, and all the leaves are at the same depth.

A $\exists_{n}$-perfect binary tree, denoted ${ }^{*} \mathcal{T}_{n}^{\exists}$, is a $\Gamma$-labeled perfect binary tree, whose root is labeled with $\exists$ and height is $(n-1)$. Likewise, a $\forall_{n}$-perfect binary tree, denoted ${ }^{*} \mathcal{T}_{n}^{\forall}$, is a $\Gamma$-labeled perfect binary tree, whose root is labeled with $\forall$ and height is $(n-1)$. 
2.3. Prefixes. The following terminology and conventions come from Grädel-McColm [2] and Rosen [6]. A prefix $p$ is a finite string in $\Gamma^{*}$. The dual of $p$, denoted by $\bar{p}$, is the prefix obtained from $p$ by swapping $\exists$ with $\forall$. Let $\mathcal{P} \subseteq \Gamma^{*}$. Then $\overline{\mathcal{P}}:=\left\{\bar{p} \in \Gamma^{*} \mid p \in \mathcal{P}\right\}$. A prefix $p$ is a subsequence of a prefix $q$ if $p$ can be obtained from $q$ by possibly deleting some elements of $q$, without changing the order of the remaining elements of $q$. A partial order on $\Gamma^{*}$, called prefix embedding, can be defined as follows: $p \preceq q$ iff $p$ is a subsequence of $q$. Here we use the curly symbol to distinguish it from the usual symbol of linear orders. Nevertheless, whether a symbol stands for a linear order or (prefix) embedding should be easily decided from the context. We use the same notation " two sets of prefixes. For $\mathcal{P}_{1}, \mathcal{P}_{2} \subseteq \Gamma^{*}, \mathcal{P}_{1} \preceq \mathcal{P}_{2} \Leftrightarrow \forall p \in \mathcal{P}_{1}, \exists q \in \mathcal{P}_{2}$ s.t. $p \preceq q$. $\mathcal{P}_{1} \prec \mathcal{P}_{2}$ if $\mathcal{P}_{1} \preceq \mathcal{P}_{2}$ but $\mathcal{P}_{2} \npreceq \mathcal{P}_{1}$. We use “*” to denote the concatenation of words. For any $\alpha \in \Gamma$ and $\mathcal{P} \subseteq \Gamma^{*}, \alpha * \mathcal{P}:=\{\alpha * p \mid p \in \mathcal{P}\}$. We define $\mathcal{P}^{-}:=\{p \mid \exists q \in \mathcal{P}$ s.t. $p \preceq q\}$ as the downward closure of $\mathcal{P}$. Let $\Gamma_{c}=\left\{\exists, \forall, \exists^{*}, \forall^{*}\right\}$ where $\exists^{*}$ and $\forall^{*}$ are characters. We interpret a word in $\Gamma_{c}^{*}$ as a regular expression. $\gamma: \Gamma_{c}^{*} \rightarrow \wp\left(\Gamma^{*}\right)$ maps such a regular expression to the regular language it denotes, where $\wp\left(\Gamma^{*}\right)$ is the power set of $\Gamma^{*}$. We define $\gamma^{-}: \Gamma_{c}^{*} \rightarrow \wp\left(\Gamma^{*}\right)$ so that for any $v \in \Gamma_{c}^{*}, \gamma^{-}(v)=\left\{q \in \Gamma^{*} \mid\right.$ there is $q^{\prime} \in \gamma(v)$ and $\left.q \preceq q^{\prime}\right\}$, the downward closure of $\gamma(v)$.

For a prefix $p,|p|$ is the length of $p . p[i]$ is the $i$-th letter of $p$. Let $l(p)$ be the last letter of $p$. For $0 \leq i<|p|$, let $p^{-i}$ be the prefix obtained from $p$ by removing the first $i$ letters in $p$, i.e. $p=p[1] * \cdots p[i] * p^{-i}$.

Finally, let $\epsilon$ be the empty string.

Lemma 2.1. Let $p, q$ be prefixes. The following hold:

(1) $\overline{\bar{p}}=p$.

(2) $\bar{p} * \bar{q}=\overline{p * q}$.

Proof.

(1) By definition.

(2) Let $|p|=n,|q|=m$.

By definition, $\bar{p} * \bar{q}=\overline{p[1] * \cdots * p[n]} * \overline{q[1] * \cdots * q[m]}=(\overline{p[1]} * \cdots * \overline{p[n]}) *(\overline{q[1]} * \cdots * \overline{q[m]})$. Because the concatenation operation on words satisfies the associative law, it follows that $(\overline{p[1]} * \cdots * \overline{p[n]}) *(\overline{q[1]} * \cdots * \overline{q[m]})=\overline{p[1]} * \cdots * \overline{p[n]} * \overline{q[1]} * \cdots * \overline{q[m]}$. Hence, by definition, $\bar{p} * \bar{q}=\bar{p} * q$.

Definition 2.2. (Rosen, [6]). Let $s \in \Gamma$ and $s^{i}$ denote the string consisting of $i$ repetitions of $s$. Define $f: \Gamma^{*} \rightarrow \Gamma_{c}^{*}$ as follows:

(1) If $p=\exists^{n}$, then $f(p):=a_{1} * \cdots * a_{2 n-1}$, where $a_{i}=\forall^{*}$ for $i$ odd, and $a_{i}=\exists$ for $i$ even;

(2) If $p=\forall^{n}$, then $f(p):=a_{1} * \cdots * a_{2 n-1}$, where $a_{i}=\exists^{*}$ for $i$ odd, and $a_{i}=\forall$ for $i$ even;

(3) If $p=s_{1}^{i_{1}} * \cdots * s_{n}^{i_{n}}\left(s_{i} \in\{\exists, \forall\}, s_{i} \neq s_{i+1}, i_{j} \in \mathbb{N}^{+}\right)$, then $f(p):=f\left(s_{1}^{i_{1}}\right) * \cdots * f\left(s_{n}^{i_{n}}\right)$.

Lemma 2.3. Let $p$ be a prefix. Then $f(p)=\overline{f(\bar{p})}$.

Proof. Assume that $p=s_{1}^{i_{1}} * \cdots * s_{n}^{i_{n}}\left(s_{i} \in\{\exists, \forall\}, s_{i} \neq s_{i+1}, i_{j} \in \mathbb{N}^{+}\right)$. By the definition of the dual of a prefix, $\bar{p}={\overline{s_{1}}}^{i} * \cdots *{\overline{s_{n}}}^{i_{n}}$. Note that $\overline{s_{i}} \neq \overline{s_{i+1}}$ since $s_{i} \neq s_{i+1}$. By definition,

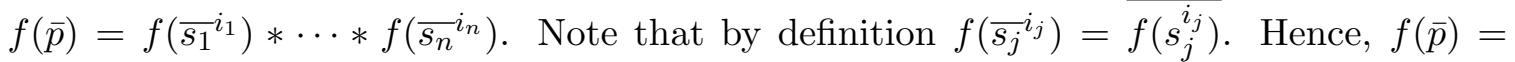
$\overline{f\left(s_{1}^{i_{1}}\right)} * \cdots * \overline{f\left(s_{n}^{i_{n}}\right)}$. By Lemma 2.1 (ii), it means that $f(\bar{p})=\overline{f\left(s_{1}^{i_{1}}\right) * \cdots * f\left(s_{n}^{i_{n}}\right)}=\overline{f(p)}$. Therefore, $f(p)=\overline{f(\bar{p})}$. 
Lemma 2.4. (Rosen, [6]). For every prefix $p \in \Gamma^{*}, f(p)$ is the unique word in $\Gamma_{c}^{*}$ such that $\gamma^{-}(f(p))=\left\{q \in \Gamma^{*} \mid p \npreceq q\right\}$.

Lemma 2.5. For any $\mathcal{P}, \mathcal{P}_{1}, \mathcal{P}_{2} \subseteq \Gamma^{*}, \mathcal{P}^{-}=\mathcal{P}_{1}^{-} \cup \mathcal{P}_{2}^{-}$if $\mathcal{P}=\mathcal{P}_{1} \cup \mathcal{P}_{2}$.

Proof. If $p \in \mathcal{P}^{-}$, then there exists $q \in \mathcal{P}_{1} \cup \mathcal{P}_{2}$ such that $p \preceq q$. In other words, either $q \in \mathcal{P}_{1}$ or $q \in \mathcal{P}_{2}$ such that $p \preceq q$. That is, $p \in \mathcal{P}_{1}^{-}$or $p \in \mathcal{P}_{2}^{-}$. Hence, $p \in \mathcal{P}_{1}^{-} \cup \mathcal{P}_{2}^{-}$.

If $p \in \mathcal{P}_{1}^{-} \cup \mathcal{P}_{2}^{-}$, then either $p \in \mathcal{P}_{1}^{-}$or $p \in \mathcal{P}_{2}^{-}$. That is, there exists $q \in \mathcal{P}_{1}$, or $q \in \mathcal{P}_{2}$, such that $p \preceq q$. In other words, there exists $q \in \mathcal{P}$ such that $p \preceq q$. Therefore, $p \in \mathcal{P}^{-}$.

2.4. Quantifier classes. Let $\varphi$ be a FO formula. Recall that we assume that any formula is in negation normal form. Let $\uplus$ be the disjoint union and $\biguplus_{i} S_{i}$ be the disjoint union of $S_{i}$.

If $\Phi$ is a set of formulas, then $\bigwedge \Phi$ ( $\bigvee \Phi$ resp.) is the conjunction (disjunction resp.) of all the formulas in $\Phi$. Similarly, we use $\bigwedge_{i} \theta_{i}\left(\bigvee_{i} \theta_{i}\right.$ resp.) to represent the conjunction (disjunction resp.) of all $\theta_{i}$.

Definition 2.6. The quantifier structure of $\varphi$, denoted $q s(\varphi)$, is a $\Gamma$-labeled forest, which is defined inductively as follows:

- If $\varphi$ is a literal, then $q s(\varphi)$ is empty;

- If $\varphi=\bigwedge_{i} \theta_{i}$ or $\bigvee_{i} \theta_{i}$, then $q s(\varphi)$ is $\biguplus_{i} q s\left(\theta_{i}\right)$;

- If $\varphi=\exists x \theta$, then $q s(\varphi)$ is composed of an $\mathscr{E}$ node and $q s(\theta)$ where there is an arc from this $\mathscr{E}$ node to each root of $q s(\theta)$ (note that $q s(\theta)$ is a forest);

Similarly, If $\varphi=\forall x \theta$, then $q s(\varphi)$ is composed of an $\mathscr{A}$ node and $q s(\theta)$ where there is an arc from this $\mathscr{A}$ node to each root of $q s(\theta)$;

In these two cases, if $q s(\theta)$ is empty, then $q s(\varphi)$ contains a single node.

Note that this definition is different from Grädel and McColm's [2], in which $q s(\varphi)$ is defined as a set of strings:

- If $\psi$ is a literal, then $q s(\psi)=\{\epsilon\}$ where $\epsilon$ is the empty word;

- If $\psi:=\bigwedge \Phi$ or $\psi:=\bigvee \Phi$ where $\Phi$ is a set of formulas, then

$$
q s(\psi):=\bigcup_{\varphi \in \Phi} q s(\varphi)
$$

- $\psi:=\exists x_{i} \varphi$, then $q s(\varphi):=\exists * q s(\varphi)$; likewise, if $\psi:=\forall x_{i} \varphi$, then $q s(\psi):=\forall * q s(\varphi)$.

Definition 2.7. Let $S_{1}, S_{2}$ be two $\Gamma$-labeled forests. Define $S_{1} \preceq_{e} S_{2}$ if there is a mapping $\iota$, not necessarily injective, from the nodes of $S_{1}$ to the nodes of $S_{2}$ such that $v$ and $\iota(v)$ have the same label for any $v$, and there is a nontrivial directed path from $\iota(x)$ to $\iota(y)$ in $S_{2}$ if there is an arc from node $x$ to node $y$ in $S_{1}$.

Remark 2.8. Note that the relation $\preceq_{e}$ is not necessary antisymmetric. That is, there are non-isomorphic $\Gamma$-labeled forests $S_{1}, S_{2}$ such that $S_{1} \preceq_{e} S_{2}$ and $S_{2} \preceq_{e} S_{1}$.

Definition 2.9. Suppose that we are given a $\Gamma$-labeled forest $S$. For any path $P:=$ $\left(v_{0}, \cdots, v_{n}\right)$ in the forest, there is a word $\left(s_{0}, \cdots, s_{n}\right)$ in $\Gamma^{*}$ associated with it such that the node $v_{i}$ is labeled with $s_{i}$. We say that this word, as well as all its subsequences, can be read off this $\Gamma$-labeled forest. Let $\mathscr{W}(\mathcal{S})$ be the set of words that can be read off the forest $\mathcal{S}$. 
Definition 2.10. Suppose that we are given a set $\mathcal{P} \subseteq \Gamma^{*}$. Let $\mathcal{P}=\mathcal{P}_{\exists} \cup \mathcal{P}_{\forall}$, where $\mathcal{P}_{\exists}=\exists * \mathcal{P}_{1}$ and $\mathcal{P}_{\forall}=\forall * \mathcal{P}_{2}$. These sets can be empty. We can inductively define a $\Gamma$-labeled forest $\mathscr{F}(\mathcal{P})$ as follows:

(1) If $\mathcal{P}$ is empty, then $\mathscr{F}(\mathcal{P})$ is empty, i.e. this forest contains no node.

(2) Let $S_{1}$ be a $\Gamma$-labeled forest such that its root is an $\mathscr{E}$ node and there is an arc from this root to each root of $\mathscr{F}\left(\mathcal{P}_{1}\right)$. Likewise, let $S_{2}$ be a $\Gamma$-labeled forest such that its root is an $\mathscr{A}$ node and there is an arc from this root to each root of $\mathscr{F}\left(\mathcal{P}_{2}\right)$.

(3) $\mathscr{F}(\mathcal{P})$ is the disjoint union of $S_{1}$ and $S_{2}$.

Note that $\mathscr{F}(P)$ is composed of at most two trees, the roots of which have different labels.

Lemma 2.11. $\mathscr{W}(\mathscr{F}(\mathcal{P}))=\mathcal{P}^{-}$, for all $\mathcal{P} \subseteq \Gamma^{*}$.

Proof. The base case when $\mathscr{F}(\mathcal{P})$ is empty, i.e. when $\operatorname{rk}(\mathscr{F}(\mathcal{P}))=0$, is trivial.

Assume that it holds when $r k(\mathscr{F}(\mathcal{P})) \leq k$ for some $k \geq 0$.

Assume that

$$
r k(\mathscr{F}(\mathcal{P}))=k+1 \text { and } \mathcal{P}=\mathcal{P}_{\exists} \cup \mathcal{P}_{\forall},
$$

where

$$
\mathcal{P}_{\exists}=\exists * \mathcal{P}_{1} \text { and } \mathcal{P}_{\forall}=\forall * \mathcal{P}_{2} .
$$

Clearly,

$$
r k\left(\mathscr{F}\left(\mathcal{P}_{1}\right)\right) \leq k \text { and } r k\left(\mathscr{F}\left(\mathcal{P}_{2}\right)\right) \leq k
$$

According to Definition 2.10, $\mathscr{F}(\mathcal{P})$ is the disjoint union of $\mathscr{F}\left(\mathcal{P}_{\exists}\right)$ and $\mathscr{F}\left(\mathcal{P}_{\forall}\right)$. In other words, $\mathscr{W}(\mathscr{F}(\mathcal{P}))$ equals $\mathscr{W}\left(\mathscr{F}\left(\mathcal{P}_{\exists}\right)\right) \cup \mathscr{W}\left(\mathscr{F}\left(\mathcal{P}_{\forall}\right)\right)$, hence equals

$$
\left(\exists * \mathscr{W}\left(\mathscr{F}\left(\mathcal{P}_{1}\right)\right)\right) \cup \mathscr{W}\left(\mathscr{F}\left(\mathcal{P}_{1}\right)\right) \cup\left(\forall * \mathscr{W}\left(\mathscr{F}\left(\mathcal{P}_{2}\right)\right)\right) \cup \mathscr{W}\left(\mathscr{F}\left(\mathcal{P}_{2}\right)\right),
$$

and by assumption equals

$$
\left(\exists * \mathcal{P}_{1}^{-}\right) \cup \mathcal{P}_{1}^{-} \cup\left(\forall * \mathcal{P}_{2}^{-}\right) \cup \mathcal{P}_{2}^{-}=\mathcal{P}_{\exists}^{-} \cup \mathcal{P}_{\forall}^{-} .
$$

By Lemma 2.5. $\mathcal{P}^{-}=\mathcal{P}_{\exists}^{-} \cup \mathcal{P}_{\forall}^{-}$. Therefore, $\mathscr{W}(\mathscr{F}(\mathcal{P}))=\mathcal{P}^{-}$.

Remark 2.12. This lemma implies that, for any $p \in \mathcal{P}^{-} \subseteq \Gamma^{*}, p$ can be read off from some path of $\mathscr{F}(\mathcal{P})$.

Lemma 2.13. For any $\Gamma$-labeled forest $S$ and $\mathcal{P} \subseteq \Gamma^{*}, S \preceq_{e} \mathscr{F}(\mathcal{P})$ if $\mathscr{W}(S) \subseteq \mathcal{P}^{-}$.

Proof. The base case when $S$ is empty is trivial.

Assume that it holds when $r k(S) \leq k$ where $k \geq 0$.

Let $S$ be a $\Gamma$-labeled forest such that $r k(S)=k+1$ and $\mathscr{W}(S) \subseteq \mathcal{P}^{-} . S$ is a disjoint union of at most two forests $S_{\exists}$ and $S_{\forall}$ : the roots of $S_{\exists}$ are all $\mathscr{E}$ nodes and the roots of $S_{\forall}$ are all $\mathscr{A}$ nodes. Then $\mathscr{W}(S)=\mathscr{W}\left(S_{\exists}\right) \cup \mathscr{W}\left(S_{\forall}\right)$. Note that a substructure of a forest is also a forest. Because $\mathscr{W}(S) \subseteq \mathcal{P}^{-}$and Lemma 2.11, $\mathscr{W}(S) \subseteq \mathscr{W}(\mathscr{F}(P))$. It means that there is a forest (substructure) $\mathcal{F}_{\exists}$ of $\mathscr{F}(P)$ such that all its roots are $\mathscr{E}$ nodes and that $\mathscr{W}\left(S_{\exists}\right) \subseteq \mathscr{W}\left(\mathcal{F}_{\exists}\right)$. Likewise, there is a forest (substructure) $\mathcal{F}_{\forall}$ of $\mathscr{F}(P)$ such that all its roots are $\mathscr{A}$ nodes and that $\mathscr{W}\left(S_{\forall}\right) \subseteq \mathscr{W}\left(\mathcal{F}_{\forall}\right)$. Note that $\mathcal{F}_{\exists}$ and $\mathcal{F}_{\forall}$ are not necessary disjoint.

Now, if we remove all the $\mathscr{E}$ roots from $S_{\exists}$, we get a forest called $S_{1}$. Similarly, if we remove all the $\mathscr{A}$ roots from $S_{\forall}$, we get another forest called $S_{2}$. 
Likewise, if we remove all the $\mathscr{E}$ roots from $\mathcal{F}_{\exists}$, we get a forest called $\mathcal{F}_{1}$. Similarly, if we remove all the $\mathscr{A}$ roots from $\mathcal{F}_{\forall}$, we get another forest called $\mathcal{F}_{2}$.

Observe that $\mathscr{W}\left(S_{1}\right) \subseteq \mathscr{W}\left(\mathcal{F}_{1}\right) \subseteq \mathscr{W}\left(\mathcal{F}_{1}\right)^{-}$and $r k\left(S_{1}\right) \leq k$. By assumption, $S_{1} \preceq_{e} \mathcal{F}_{1}$. Let us denote the map that embeds $S_{1}$ to $\mathcal{F}_{1}$ as $\iota_{1}$. Likewise, $S_{2} \preceq_{e} \mathcal{F}_{2}$ and the embedding map is denoted $\iota_{2}$. Note that the domains of $\iota_{1}$ and $\iota_{2}$ are different. Therefore, we can merge these two maps easily, i.e. let $\iota_{0}=\iota_{1} \cup \iota_{2}$. Note that $S_{1}\left(S_{2}\right.$ resp.) is embeddable to $\mathcal{F}_{1}\left(\mathcal{F}_{2}\right.$ resp.) through $\iota_{0}$. Now, we can extend the embedding map $\iota_{0}$ to $\iota$ such that: (i) the father of any root $r_{1}$ of $S_{1}$ is mapped to the father of $\iota_{0}\left(r_{1}\right)$; (ii) the father of any root $r_{2}$ of $S_{2}$ is mapped to the father of $\iota_{0}\left(r_{2}\right)$. Therefore, $S$ is embeddable to $\mathscr{F}(P)$ through $\iota$, i.e. $S \preceq_{e} \mathscr{F}(P)$.

Remark 2.14. Lemma 2.11 and Lemma 2.13 tell us that $\mathscr{F}(P)$ is the "maximal" $\Gamma$-labeled forest (in the sense of embeddings) among all these forests, from which the set of words that can be read off is a subset of $\mathcal{P}^{-}$.

Define the quantifier rank of FO formula $\varphi$, denoted $q r(\varphi)$, to be $r k(q s(\varphi))$. Note that this definition is equivalent to the usual definition of quantifier rank (see for instance Libkin [4]). Let $\mathrm{FO}[k]:=\{\varphi \in \mathrm{FO} \mid \operatorname{qr}(\varphi) \leq k\}$.

Let $S$ be a $\Gamma$-labeled forest. Define the quantifier class FO $\{S\}$ to be the set of queries that are definable by the set of first-order sentences $\left.\{\theta \in \mathrm{FO} \mid q s(\theta)) \preceq_{e} S\right\}$.

A first-order formula is in prenex normal form if it is a single string of quantifiers followed by a quantifier free formula. Its quantifier prefix, which is obtained from this string of quantifiers by removing the variables in the string, corresponds to a $\Gamma$-labeled degenerate tree.

Given a prefix $p$, we define the prefix class $\mathrm{FO}(p)$ as the set of $\mathrm{FO}$ sentences in prenex normal form such that for any $\psi \in \mathrm{FO}(p)$, its prefix is a subsequence of $p$ (Grädel and McColm, 2] ). Grädel-McColm's conjecture says that the prefix classes form a strict hierarchy: For any prefix $p, q, \mathrm{FO}(p) \nsubseteq \mathrm{FO}(q)$ if $p \npreceq q$ over arbitrary structures. Rosen [6] confirmed this conjecture over infinite structures and called it the first-order prefix hierarchy. Similarly, we can define a hierarchy formed by quantifier classes, which can be called the first-order quantifier structure hierarchy. These two hierarchies are independent.

\section{Quantifier Structure Hierarchy: the first observation}

In this section, we define a variant of Ehrenfeucht-Fraïssé games that characterizes quantifier classes and prove that those quantifier classes form a natural and strict hierarchy:

Theorem 3.1. Let $S_{1}$ and $S_{2}$ be two $\Gamma$-labelled forests. Over the class of all digraphs,

$$
\text { if } \mathscr{W}\left(S_{1}\right) \nsubseteq \mathscr{W}\left(S_{2}\right) \text {, then } \mathrm{FO}\left\{S_{1}\right\} \nsubseteq \mathrm{FO}\left\{S_{2}\right\} \text {. }
$$

3.1. Games that characterize quantifier classes. Let $S$ be a $\Gamma$-labelled forest. We define an asymmetric variant of the Ehrenfeucht-Fraïssé games as follows. Let $k \in \mathbb{N}, \sigma$ contains $k$ constant symbols. Let $\mathfrak{A}$ and $\mathfrak{B}$ be two $\sigma$-structures. Let the $k$-tuple $\overline{\mathfrak{u}}$ be the interpretation of the constants in $\mathfrak{A}$ and the $k$-tuple $\overline{\mathfrak{v}}$ be the interpretation of the constants in $\mathfrak{B}$. The game $G_{S}(\mathfrak{A}, \mathfrak{B})$ is played by two players, called the spoiler and duplicator, on a game board consisting of $S, \mathfrak{A}$ and $\mathfrak{B}$. At the beginning of the game, the spoiler picks a tree $\mathcal{T}$ in the forest $S$ and puts a token on the root of $\mathcal{T}$. Assume that the depth of $\mathcal{T}$ is $n-1$. Afterwards, for every $i$ where $1 \leq i \leq n$, in the $i$-th round the spoiler chooses an 
element from the structure $\mathfrak{A}$ if the current node, on which the token is put, is an $\mathscr{E}$ node. Otherwise if it is an $\mathscr{A}$ node he picks an element of $\mathfrak{B}$. Then the duplicator has to respond by picking an element from the other structure. Afterwards the spoiler chooses a child of the current node in $S$ and moves the token to it. This completes one round.

Assume that after $n^{\prime}\left(n^{\prime} \leq n\right)$ rounds a sequence $\bar{c}=\left(c_{1}, \cdots, c_{n^{\prime}}\right)$ has been picked in $\mathfrak{A}$ and a sequence $\bar{d}=\left(d_{1}, \cdots, d_{n^{\prime}}\right)$ has been picked in $\mathfrak{B}$. The spoiler wins the game if $(\overline{\mathfrak{u}} \bar{c}, \overline{\mathfrak{v}} \bar{d})$ does not define a partial isomorphism between $\mathfrak{A}$ and $\mathfrak{B}$.

The game ends whenever the spoiler wins or the token arrives at a leaf of $\mathcal{T}$. The duplicator wins if the spoiler fails to win in the end.

Informally, $\overline{\mathfrak{u}}$ and $\overline{\mathfrak{v}}$ can be regarded as a carry-over of past history of the game played before the beginning, which has to be taken care of.

A strategy of the duplicator is a scheme by which she knows how to choose an element in each round depending on the history of the play.

For any tuple $\left(\mathfrak{a}_{1}, \cdots, \mathfrak{a}_{n}\right) \in(|\mathfrak{A}| \uplus|\mathfrak{B}|)^{n}$, we associate it with a prefix $(p[1], \cdots, p[n])$ such that $\mathfrak{a}_{i} \in|\mathfrak{A}|$ iff $p[i]=\exists$ for any $1 \leq i \leq n$. A strategy of the duplicator in the game $G_{S}(\mathfrak{A}, \mathfrak{B})$ is a function

$$
D_{\mathfrak{A}, \mathfrak{B}}^{S}: \biguplus_{i=1}^{h(S)+1}(|\mathfrak{A}| \uplus|\mathfrak{B}|)^{i} \rightarrow(|\mathfrak{A}| \uplus|\mathfrak{B}|) .
$$

If the duplicator has a strategy guiding her choices in the game that ensures her winning in the end no matter how the spoiler plays, we call this strategy a winning strategy of the duplicator. If there exists such a winning strategy for the duplicator in the game $G_{S}(\mathfrak{A}, \mathfrak{B})$ then we write $\mathfrak{A} \rightsquigarrow_{S} \mathfrak{B}$. The winning strategy of the spoiler can be defined dually because in our games either the spoiler or the duplicator has a winning strategy. Let $\bar{a} \in|\mathfrak{A}|^{t}$ and $\bar{b} \in|\mathfrak{B}|^{t}$. We use $(\mathfrak{A}, \bar{a}) \rightsquigarrow_{S}(\mathfrak{B}, \bar{b})$ to denote that the duplicator has a winning strategy, in which $\bar{a}$ is picked in $\mathfrak{A}$, and $\bar{b}$ is picked in $\mathfrak{B}$, before the game starts. Equivalently, we say that the spoiler has a winning strategy in the game $G_{S}((\mathfrak{A}, \bar{a}),(\mathfrak{B}, \bar{b}))$.

Note that the standard Ehrenfeucht-Fraïssé game $G_{n}(\mathfrak{A}, \mathfrak{B})$ (see [4]) is exactly the game $G_{S}(\mathfrak{A}, \mathfrak{B})$ where $S=\left\{{ }^{*} \mathcal{T}_{n}^{\exists},{ }^{*} \mathcal{T}_{n}^{\forall}\right\}$.

Definition 3.2. Let $n \in \mathbb{N}$ and $\bar{c}$ be an $n$-tuple of elements from $|\mathfrak{A}|$. Then for a $\Gamma$-labelled forest $S$, the $Q S$-S $n$-type of $\bar{c}$ over $\sigma$-structure $\mathfrak{A}$ is defined as:

$$
t p_{n}^{S}(\mathfrak{A}, \bar{c})=\{\varphi(\bar{c}) \in \operatorname{FO}\{S\} \mid \mathfrak{A} \models \varphi(\bar{c})\} .
$$

The following lemma is well-known, cf. [4] for a simple explanation.

Lemma 3.3. For fixed $k, n \in \mathbb{N}$, there are only finitely many formulas, in $n$ free variables, in $\mathrm{FO}[k]$ up to logical equivalence.

Corollary 3.4. Let $n \in \mathbb{N}$ and $S$ be a $\Gamma$-labelled forest, and let $\bar{c}$ be an $n$-tuple of elements from $|\mathfrak{A}|$, there are only finitely many formulas in $t p_{n}^{S}(\mathfrak{A}, \bar{c})$ up to logical equivalence.

Let $S$ be a $\Gamma$-labelled forest.

Definition 3.5. Let $[\mathfrak{A}, \bar{a}]$ be an expansion of $\mathfrak{A}$ to $\sigma \cup\{\overline{\mathfrak{c}}\}$ such that $\bar{a}$ interprets the tuple of constants $\overline{\mathfrak{c}}$ in $[\mathfrak{A}, \bar{a}]$.

Lemma 3.6. Let $S$ and $S^{\prime}$ be two $\Gamma$-labelled forests such that $S^{\prime} \preceq_{e} S$. Let $\mathfrak{A}$ and $\mathfrak{B}$ be two structures over the same signature. If the duplicator has a winning strategy in the game $G_{S}(\mathfrak{A}, \mathfrak{B})$, then she also has a winning strategy in the game $G_{S^{\prime}}(\mathfrak{A}, \mathfrak{B})$. 
Proof. Note that the duplicator can mimic her winning strategy in the game $G_{S^{\prime}}(\mathfrak{A}, \mathfrak{B})$ to play the game $G_{S^{\prime}}(\mathfrak{A}, \mathfrak{B})$. And a subset of a partial isomorphism is still a partial isomorphism.

Remark 3.7. Lemma 3.6 tells us that if the duplicator has a winning strategy in $G_{S}(\mathfrak{A}, \mathfrak{B})$, she also has a winning strategy when the players are allowed to skip playing arbitrary rounds of the game. Lemma 3.6 also tells us that if the spoiler has a winning strategy in $G_{S^{\prime}}(\mathfrak{A}, \mathfrak{B})$, he also has a winning strategy in $G_{S}(\mathfrak{A}, \mathfrak{B})$. In other words, quantifiers are logical resources that can be exploited by the spoiler to detect the difference between two structures in the games.

It is obvious that $[\mathfrak{A}, \bar{a}] \rightsquigarrow_{S}[\mathfrak{B}, \bar{b}]$ iff $(\mathfrak{A}, \bar{a}) \rightsquigarrow_{S}(\mathfrak{B}, \bar{b})$, because in each round of both the game $G_{S}([\mathfrak{A}, \bar{a}],[\mathfrak{B}, \bar{b}])$ and $G_{S}((\mathfrak{A}, \bar{a}),(\mathfrak{B}, \bar{b}))$, if there is a partial isomorphism between two structures in the former, then this partial isomorphism is also a partial isomorphism between two structures in the latter.

In the following, we prove a connection between the games just defined and quantifier classes, which is a variant of the result of Grädel and McColm [2].

Recall that $\mathfrak{A}$ has constants that are interpreted by $\overline{\mathfrak{u}}$. And $\mathfrak{B}$ has constants that are interpreted by $\overline{\mathfrak{v}}$. We assume that $\overline{\mathfrak{u}}$ ( $\overline{\mathfrak{v}}$ resp.) and $\bar{a}(\bar{b}$ resp.) do not share any element.

Theorem 3.8. For arbitrary finite $\sigma$-structures $\mathfrak{A}, \mathfrak{B}$, two tuples $\bar{a} \in|\mathfrak{A}|^{t}, \bar{b} \in|\mathfrak{B}|^{t}$, and $a$ $\Gamma$-labelled forest $S$, the following are equivalent:

(i) $[\mathfrak{A}, \bar{a}] \rightsquigarrow S[\mathfrak{B}, \bar{b}]$;

(ii) $t p_{t}^{S}(\mathfrak{A}, \bar{a}) \subseteq t p_{t}^{S}(\mathfrak{B}, \bar{b})$.

Proof. (i) $\rightarrow$ (ii):

When $S$ is an empty forest, i.e. $r k(S)=0, t p_{t}^{S}(\mathfrak{A}, \bar{a}) \nsubseteq t p_{t}^{S}(\mathfrak{B}, \bar{b})$ means there is a quantifier-free formula $\eta(\bar{x})$ such that $(\mathfrak{A}, \bar{a}) \models \eta(\bar{x})$ but $(\mathfrak{B}, \bar{b}) \not \models \eta(\bar{x})$. Hence, the mapping from $\overline{\mathfrak{u}} \bar{a}$ to $\overline{\mathfrak{v}} \bar{b}$ does not define a partial isomorphism. In other words, the spoiler wins the game and $[\mathfrak{A}, \bar{a}] \not \varkappa_{S}[\mathfrak{B}, \bar{b}]$.

Assume that (i) $\rightarrow$ (ii) when $r k(S) \leq k$ for $k \geq 0$.

Assume that $r k(S)=k+1$ and $S$ consists of $m$ trees $S_{1}, \cdots, S_{m}$. Suppose that $(i i)$ is false. Let $\varphi(\bar{x}) \in \operatorname{FO}\{S\}$ such that $(\mathfrak{A}, \bar{a}) \models \varphi(\bar{x})$ but $(\mathfrak{B}, \bar{b}) \not \models \varphi(\bar{x})$. Then $\varphi$ is a first-order formula that is a disjunction or conjunction of formulas $\mathrm{FO}\left\{S_{i}\right\}(1 \leq i \leq m)$. There must exist one disjunct or conjunct $\psi$ such that $(\mathfrak{A}, \bar{a}) \models \psi(\bar{x})$ while $(\mathfrak{B}, \bar{b}) \not \models \psi(\bar{x})$, where $q s(\psi) \preceq{ }_{e} S_{i}$ for some $1 \leq i \leq m$. By Lemma 3.6, we may assume that the spoiler moves the token from the root of $S_{i}$. Assume that the root of $S_{i}$ is an $\mathscr{E}$ node, then $\psi$ has the form $\exists y \psi_{1}(\bar{x} y)$. Hence, there is $c \in|\mathfrak{A}|$ s.t. $(\mathfrak{A}, \bar{a} c) \models \psi_{1}(\bar{x} y)$. Then the spoiler can pick $c$, and no matter which element, say $d$, the duplicator picks, $\psi_{1}(\bar{x} y)$ distinguishes the pair $[\mathfrak{A}, \bar{a} c]$ and $[\mathfrak{B}, \bar{b} d]$, where the variables $\bar{x} y$ are assigned the values $\bar{a} c$ and $\bar{b} d$ respectively, because $(\mathfrak{B}, \bar{b}) \not \forall \exists y \psi_{1}(\bar{x} y)$. By induction assumption the spoiler has a winning strategy over the game $G_{q s\left(\psi_{1}\right)}([\mathfrak{A}, \bar{a} c],[\mathfrak{B}, \bar{b} d])$. Similarly, if $S_{i}$ is a tree whose root is an $\mathscr{A}$ node, the spoiler can pick $d \in|\mathfrak{B}|$ such that for any $c \in|\mathfrak{A}|$ picked by the duplicator $\psi_{1}(\bar{x} y)$ distinguishes the pair $[\mathfrak{A}, \bar{a} c]$ and $[\mathfrak{B}, \bar{b} d]$ where the variables $\bar{x} y$ are assigned the values $\bar{a} c$ and $\bar{b} d$ respectively. In other words, the spoiler can show that there is an element $d$ such that it makes $(\mathfrak{B}, \bar{b} d) \not \neq \psi_{1}(\bar{x} y)$ while $(\mathfrak{A}, \bar{a} c) \mid=\psi_{1}(\bar{x} y)$ is always true. By induction assumption the spoiler has a winning strategy over the game $G_{q s\left(\psi_{1}\right)}([\mathfrak{A}, \bar{a} c],[\mathfrak{B}, \bar{b} d])$. Therefore, $[\mathfrak{A}, \bar{a}] \varkappa_{S}[\mathfrak{B}, \bar{b}]$.

(ii) $\rightarrow(\mathrm{i})$ : 
According to the definition of the game, when $r k(S)=0,[\mathfrak{A}, \bar{a}] \not \varkappa_{S}[\mathfrak{B}, \bar{b}]$ means that the mapping from $\overline{\mathfrak{u}} \bar{a}$ to $\overline{\mathfrak{v}} \bar{b}$ is not a partial isomorphism, which implies that there exists a quantifier-free FO formula $\xi(\bar{x})$ s.t. either $((\mathfrak{A}, \bar{a})=\xi(\bar{x})$ but $(\mathfrak{B}, \bar{b}) \not \models \xi(\bar{x}))$, or $((\mathfrak{A}, \bar{a}) \not \models \xi(\bar{x})$ but $(\mathfrak{B}, \bar{b}) \models \xi(\bar{x}))$. Let $\neg \xi(\bar{x})$ be the negation of $\xi(\bar{x})$. In the former case, it implies that $t p_{t}^{S}(\mathfrak{A}, \bar{a}) \nsubseteq t p_{t}^{S}(\mathfrak{B}, \bar{b})$; in the latter case, $(\mathfrak{A}, \bar{a}) \models \neg \xi(\bar{x})$ but $(\mathfrak{B}, \bar{b}) \not \neg \neg \xi(\bar{x})$, which also implies that $t p_{t}^{S}(\mathfrak{A}, \bar{a}) \nsubseteq t p_{t}^{S}(\mathfrak{B}, \bar{b})$. All in all, $[\mathfrak{A}, \bar{a}] \varkappa_{S}[\mathfrak{B}, \bar{b}] \operatorname{implies} t p_{t}^{S}(\mathfrak{A}, \bar{a}) \nsubseteq t p_{t}^{S}(\mathfrak{B}, \bar{b})$ when $r k(S)=0$.

Assume that (ii) $\rightarrow$ (i) when $r k(S)=k$ for $k \geq 0$.

Now assume that $S$ is composed of trees $S_{1}, \cdots, S_{m}$ and $r k(S)=k+1$. Assume that $(i)$ is false. Then over one of the trees the spoiler has a winning strategy. Hence, the spoiler can first pick this tree to play. If this tree's root is an $\mathscr{E}$ node $r$, we can regard this tree as a digraph composed of $r$ and a forest $S^{\prime}$ such that there is an arc from $r$ to each root of $S^{\prime}$. In the first round the spoiler can pick an element $c \in|\mathfrak{A}|$ such that no matter which element $d \in|\mathfrak{B}|$ the duplicator picks, $[\mathfrak{A}, \bar{a} c]$ and $[\mathfrak{B}, \bar{b} d]$ form the new game board over which the spoiler will win the game $G_{S^{\prime}}([\mathfrak{A}, \bar{a} c],[\mathfrak{B}, \bar{b} d])$. By Corollary 3.4 , there are only finitely many formulas in $t p_{t+1}^{S^{\prime}}(\mathfrak{A}, \bar{a} c)$ up to logical equivalence. Let $T / E$ be a set of formulas where each equivalent class in $t p_{t+1}^{S^{\prime}}(\mathfrak{A}, \bar{a} c)$ has exactly one formula in $T / E$. Let $\varphi(\bar{x} y)$ be the conjunction of all the formulas in $T / E$. By the induction hypothesis, for any $d$ there is a formula $\eta(\bar{x} y) \in \mathrm{FO}\left\{S^{\prime}\right\}$ such that $(\mathfrak{A}, \bar{a} c) \models \eta(\bar{x} y)$ but $(\mathfrak{B}, \bar{b} d) \not \models \eta(\bar{x} y)$. Note that $\eta$ is equivalent to one formula in $T / E$. Hence, $(\mathfrak{A}, \bar{a} c) \models \varphi(\bar{x} y)$ but $(\mathfrak{B}, \bar{b} d) \not \varphi(\bar{x} y)$, for any $d$. In other words, $(\mathfrak{A}, \bar{a}) \mid=\exists y \varphi(\bar{x} y)$ but $(\mathfrak{B}, \bar{b}) \not \forall \exists y \varphi(\bar{x} y)$. Note that $\exists y \varphi(\bar{x} y) \in \mathrm{FO}\{S\}$. Therefore, $t p_{t}^{S}(\mathfrak{A}, \bar{a}) \nsubseteq t p_{t}^{S}(\mathfrak{B}, \bar{b})$.

The case when the tree picked by the spoiler in the first step is a tree whose root is an $\mathscr{A}$ node can be proved similarly.

Corollary 3.9. Let $\mathcal{K}$ be a class of finite structures and $S$ be a $\Gamma$-labelled forest. If there is $\mathfrak{A} \in \mathcal{K}$ and $\mathfrak{B} \notin \mathcal{K}$ such that $\mathfrak{A} \rightsquigarrow S \mathfrak{B}$, then there is no first-order sentence $\varphi$ such that $q s(\varphi) \preceq_{e} S$ and $\mathcal{K}=\operatorname{Mod}(\varphi)$.

\subsection{Point-expansions.}

Definition 3.10. Let $\mathfrak{A}$ be a structure over signature $\sigma_{A}$ and $\mathcal{K}$ be a set $\left\{\mathfrak{C}_{i}\right\}_{i \in I}$ of finite structures indexed by a set $I$. Let $\sigma_{i}$ be the signature of $\mathfrak{C}_{i}$ for each $i \in I$ such that $\sigma_{i}$ contains a special constant $c_{i}$ that is called hook. Assume that no two signatures share a constant. Let $\beth_{\mathfrak{A}}:|\mathfrak{A}| \rightarrow \mathcal{K}$ be a total function. Define the point-expansion of $\mathfrak{A}$ by $\beth_{\mathfrak{A}}$ over $\mathcal{K}$, denoted $\mathcal{E}_{\mathcal{K}}^{\beth_{\mathfrak{K}}}(\mathfrak{A})$, as follows:

(1) The signature of $\mathcal{E}_{\mathcal{K}}^{\beth_{\mathfrak{A}}}(\mathfrak{A})$, denoted $\sigma_{\mathcal{E}}$, is composed of the union of $\sigma_{i}$ and $\sigma_{A}$, except for the hook in $\sigma_{i}$ for any $i$.

(2) Let $M:=\biguplus_{a \in|\mathfrak{A}|} \beth_{\mathfrak{A}}(a)$ and $\left|\mathcal{E}_{\mathcal{K}}^{\beth_{\mathfrak{A}}}(\mathfrak{A})\right|:=|M|$.

(3) Let $M^{\prime}$ be the set of elements that interpret the hooks in respective disjoint substructures.

(4) There is a bijection $g: M^{\prime} \rightarrow|\mathfrak{A}|$ such that for any $k$-tuple $\bar{v}=\left(v_{1}, \cdots, v_{k}\right) \in\left|M^{\prime}\right|^{k}$ and any $k$-ary relation $R \in \sigma_{A}$,

$$
\bar{v} \in R^{\mathcal{E}_{\mathcal{K}}^{\mathcal{I}_{\mathfrak{A}}}(\mathfrak{A})} \text { iff }\left(g\left(v_{1}\right), \cdots, g\left(v_{k}\right)\right) \in R^{\mathfrak{A}} .
$$


In other words, $M^{\prime}$ induces in $\mathcal{E}_{\mathcal{K}}^{\beth_{\mathfrak{A}}}(\mathfrak{A})$ an isomorphic copy of $\mathfrak{A}$. Let

$$
R_{g}:=\left\{\bar{v} \in\left|M^{\prime}\right|^{k} \mid\left(g\left(v_{1}\right), \cdots, g\left(v_{k}\right)\right) \in R^{\mathfrak{A}}\right\} .
$$

(5) Let $\left\{\mathcal{X}_{i}^{\hat{\mathcal{R}}}\right\}$ be the set of structures in $M$ whose signatures contain $\hat{\mathcal{R}}$.

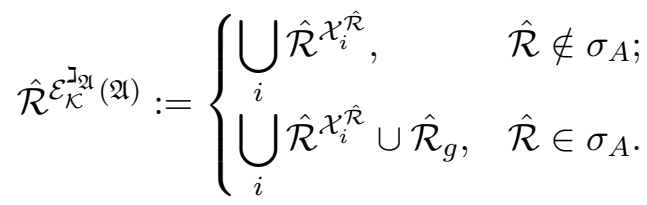

(6) For any constant $c \in \sigma_{\mathcal{E}}$,

$$
c^{\mathcal{E}^{\mathfrak{J}_{\mathfrak{K}}}(\mathfrak{A})}:= \begin{cases}c^{\mathfrak{C}_{i}}, & c \in \sigma_{i} \backslash \sigma_{A} ; \\ g^{-1}\left(c^{\mathfrak{A}}\right), & c \in \sigma_{A} .\end{cases}
$$

Informally speaking, $\mathcal{E}_{\mathcal{K}}^{\beth_{\mathfrak{A}}}(\mathfrak{A})$ is a structure that is obtained from $\mathfrak{A}$ by substituting each element $a \in|\mathfrak{A}|$ with $\beth_{\mathfrak{A}}(a)$, identifying $a$ with the hook in $\beth_{\mathfrak{A}}(a)$. A point-expansion of the structure $\mathfrak{A}$ can also be regarded as the result of a process that "glues" a small substructure $\beth_{\mathfrak{A}}(a)$ at each element $a$ (also at the hook of $\beth_{\mathfrak{A}}(a)$ ) of the "prototype" structure $\mathfrak{A}$. And each small substructure shares only one element with the prototype structure, i.e. the "point" where they are "glued" together.

Lemma 3.11. Suppose that we are given a forest $\mathcal{F}$, two structures $\mathfrak{A}, \mathfrak{B}$, a finite set $\mathcal{K}$ of structures and two mappings $\beth_{\mathfrak{A}}$ and $\beth_{\mathfrak{B}}$ that expand $\mathfrak{A}$ and $\mathfrak{B}$ over $\mathcal{K}$ respectively. Then the duplicator has a winning strategy in the game $G_{\mathcal{F}}\left(\mathcal{E}_{\mathcal{K}}^{\beth_{\mathfrak{A}}}(\mathfrak{A}), \mathcal{E}_{\mathcal{K}}^{\beth_{\mathfrak{B}}}(\mathfrak{B})\right)$ if the following is true: the duplicator has a winning strategy $D_{\mathfrak{A}, \mathfrak{B}}^{\mathcal{F}}$ in the game $G_{\mathcal{F}}(\mathfrak{A}, \mathfrak{B})$ such that

(1) for any $a \in|\mathfrak{A}|$ and sequence of elements $\bar{s} \in(|\mathfrak{A}| \uplus|\mathfrak{B}|)^{|\bar{s}|}(|\bar{s}| \leq h(\mathcal{F}))$, the duplicator has a winning strategy

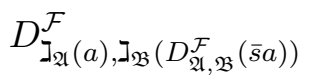

in the game $G_{\mathcal{F}}\left(\beth_{\mathfrak{A}}(a), \beth_{\mathfrak{B}}\left(D_{\mathfrak{A}, \mathfrak{B}}^{\mathcal{F}}(\bar{s} a)\right)\right)$.

(2) for any $b \in|\mathfrak{B}|$ and sequence of elements $\bar{s} \in(|\mathfrak{A}| \uplus|\mathfrak{B}|)^{|\bar{s}|}(|\bar{s}| \leq h(\mathcal{F}))$, the duplicator has a winning strategy

$$
D_{\beth_{\mathfrak{A}}}^{\mathcal{F}}\left(D_{\mathfrak{A}, \mathfrak{B}}^{\mathcal{F}}(\bar{s} b)\right), \beth_{\mathfrak{B}}(b)
$$

in the game $G_{\mathcal{F}}\left(\beth_{\mathfrak{A}}\left(D_{\mathfrak{A}, \mathfrak{B}}^{\mathcal{F}}(\bar{s} b)\right), \beth_{\mathfrak{B}}(b)\right)$.

Proof. One winning strategy of the duplicator in the game $G_{\mathcal{F}}\left(\mathcal{E}_{\mathcal{K}}^{\beth_{\mathfrak{A}}}(\mathfrak{A}), \mathcal{E}_{\mathcal{K}}^{\beth_{\mathfrak{B}}}(\mathfrak{B})\right)$ is the composition of her winning strategies in $G_{\mathcal{F}}(\mathfrak{A}, \mathfrak{B})$ and $G_{\mathcal{F}}\left(\mathfrak{C}_{i}^{A}, \mathfrak{C}_{j}^{B}\right)$ where $\mathfrak{C}_{i}^{A}, \mathfrak{C}_{j}^{B} \in \mathcal{K}$.

Let $\mathfrak{A}_{\mathfrak{I}_{\mathfrak{A}}}$ be the isomorphic copy of $\mathfrak{A}$ in $\mathcal{E}_{\mathcal{K}}^{\mathcal{I}_{\mathfrak{A}}}(\mathfrak{A})$, whose elements interpret the hooks of disjoint substructures in $\mathcal{K}$. And let $\mathfrak{B}_{\beth_{\mathfrak{B}}}$ be the isomorphic copy of $\mathfrak{B}$ in $\mathcal{E}_{\mathcal{K}}^{\beth_{\mathfrak{B}}}(\mathfrak{B})$, whose elements interpret the hooks of disjoint substructures in $\mathcal{K}$. Assume that the spoiler has already picked a sequence $\bar{s}$ of elements in the game $G_{\mathcal{F}}\left(\mathfrak{A}_{\mathfrak{I}_{\mathfrak{H}}}, \mathfrak{B}_{\mathfrak{I}_{\mathfrak{B}}}\right)$ :

(ii) When the spoiler picks an element in $\mathfrak{A}_{\beth_{\mathfrak{A}}}$ or $\mathfrak{B}_{\mathfrak{I}_{\mathfrak{B}}}$, the duplicator uses the strategy $D_{\mathfrak{A}, \mathfrak{B}}^{\mathcal{F}}$; otherwise:

(iii) For any $a \in\left|\mathfrak{A}_{\mathfrak{A}}\right|$, if the spoiler picks an element in $\beth_{\mathfrak{A}}(a)$, the duplicator uses the strategy $D_{\beth_{\mathfrak{A}}(a), \beth_{\mathfrak{B}}\left(D_{\mathfrak{A}, \mathfrak{B}}^{\mathcal{F}}(\bar{s} a)\right)}^{\mathcal{F}}$; 
(iiii) For any $b \in\left|\mathfrak{B}_{\beth_{\mathfrak{B}}}\right|$, if the spoiler picks an element in $\boldsymbol{I}_{\mathfrak{B}}(b)$, the duplicator uses the strategy $D_{\mathfrak{I}_{\mathfrak{A}}\left(D_{\mathfrak{A}, \mathfrak{B}}^{\mathcal{F}}(\bar{s} b)\right), \beth_{\mathfrak{B}}(b)}^{\mathcal{F}}$.

Clearly, partial isomorphisms are preserved under such compositions, which provides the duplicator a winning strategy in the game $G_{\mathcal{F}}\left(\mathcal{E}_{\mathcal{K}}^{\mathcal{I}_{\mathfrak{A}}}(\mathfrak{A}), \mathcal{E}_{\mathcal{K}}^{\beth_{\mathfrak{B}}}(\mathfrak{B})\right)$, which can be regarded as a main game together with a series of subgames.

\subsection{Strictness of the FO quantifier structure hierarchy.}

Definition 3.12. Let $\mathcal{I}$ be a structure over signature $\left\langle c_{I}\right\rangle$ where $c_{I}$ is the hook constant and $\mathcal{I}$ has only one element, which is used to interpret $c_{I}$.

Definition 3.13. Let $m \in \mathbb{N}^{+}$and $p \in \Gamma^{*}$. Let $\tau^{+}=\langle U, R, B, r\rangle$ where $R, B$ are binary relation symbols, $U$ is a unary relation symbol, and $r$ is a constant symbol. To make it vivid, we say that an element $x$ is black if $x \in U$. All the elements in the structures are white unless explicitly labeled black. Likewise, an arc $(x, y)$ is red if $(x, y) \in R$; an arc is blue if $(x, y) \in B$. Let $\tau_{0}^{+}=\tau^{+} \backslash\{U\}$.

We define $\widetilde{\mathfrak{A}}_{m}^{p}$ and $\widetilde{\mathfrak{B}}_{m}^{p}$ to be $\tau^{+}$-structures as follows:

- $\widetilde{\mathfrak{A}}_{m}^{p}$ and $\widetilde{\mathfrak{B}}_{m}^{p}$ are trees, whose edges are either red or blue. The constant $r$ is interpreted by the root of the respective trees.

- When $|p|=1$, all edges in $\widetilde{\mathfrak{A}}_{m}^{p}$ and $\widetilde{\mathfrak{B}}_{m}^{p}$ are red.

- If $p=\exists$,

(1) $\widetilde{\mathfrak{A}}_{m}^{p}$ is a depth 1 tree that has $2 m+1$ leaves. One of its leaves is black.

(2) $\widetilde{\mathfrak{B}}_{m}^{p}$ is a depth 1 tree that has $2 m$ leaves. None of them is black.

$\mathcal{T}_{\exists, m}^{\mathfrak{A}}:=\widetilde{\mathfrak{A}}_{m}^{\exists}\left|\tau_{0}^{+} ; \mathcal{T}_{\exists, m}^{\mathfrak{B}}:=\widetilde{\mathfrak{B}}_{m}^{\exists}\right| \tau_{0}^{+}$.

- If $p=\forall$,

(1) $\widetilde{\mathfrak{A}}_{m}^{p}$ is a depth 1 tree that has $2 m$ leaves. All of them are black.

(2) $\widetilde{\mathfrak{B}}_{m}^{p}$ is a depth 1 tree that has $2 m+1$ leaves. All are black except one.

$\mathcal{T}_{\forall, m}^{\mathfrak{A}}:=\widetilde{\mathfrak{A}}_{m}^{\forall}\left|\tau_{0}^{+} ; \mathcal{T}_{\forall, m}^{\mathfrak{B}}:=\widetilde{\mathfrak{B}}_{m}^{\forall}\right| \tau_{0}^{+}$.

Note that $\mathcal{T}_{\exists, m}^{\mathfrak{A}}=\mathcal{T}_{\forall, m}^{\mathfrak{B}}$ and $\mathcal{T}_{\exists, m}^{\mathfrak{B}}=\mathcal{T}_{\forall, m}^{\mathfrak{A}}$.

- When $|p|>1$ :

Let $\widetilde{\mathfrak{A}}_{m}^{-q}$ be the same as $\widetilde{\mathfrak{A}}_{m}^{q}$ except that the colours of all the edges are exchanged, i.e. red is interchanged with blue. Let $\mathfrak{D}_{q, m}^{A, A}$, a $\left(\tau^{+} \cup\{\mathfrak{e}\} \backslash\{r\}\right)$-structure, be built from a copy of $\widetilde{\mathfrak{A}}_{m}^{q}$ and a copy of $\widetilde{\mathfrak{A}}_{m}^{-q}$ where they are joined together at their roots. Call their shared root a junction point, which interprets the hook constant $\mathfrak{e}$. Similarly, define $\mathfrak{D}_{q, m}^{A, B}$ to be the join of $\widetilde{\mathfrak{A}}_{m}^{q}$ and $\widetilde{\mathfrak{B}}_{m}^{-q}, \mathfrak{D}_{q, m}^{B, A}$ be the join of $\widetilde{\mathfrak{B}}_{m}^{q}$ and $\widetilde{\mathfrak{A}}_{m}^{-q} ; \mathfrak{D}_{q, m}^{B, B}$ be the join of $\widetilde{\mathfrak{B}}_{m}^{q}$ and $\widetilde{\mathfrak{B}}_{m}^{-q}$. By "copies" we mean disjoint copies. Let $\mathcal{K}:=\left\{\mathcal{I}, \mathfrak{D}_{q, m}^{A, A}, \mathfrak{D}_{q, m}^{A, B}, \mathfrak{D}_{q, m}^{B, A}\right\}$.

- If $p=\exists q$,

$\left(1^{\exists}\right) \widetilde{\mathfrak{A}}_{m}^{p}$ is a point-expansion of $\mathcal{T}_{\exists, m}^{\mathfrak{A}}$ over $\mathcal{K}$ as follows: The root of $\mathcal{T}_{\exists, m}^{\mathfrak{A}}$ is expanded

by $\mathcal{I}$. It is also called the "root" of $\mathcal{T}_{\exists, m}^{\mathfrak{A}}$ that interprets $r$. One of its leaves is expanded by a copy of $\mathfrak{D}_{q, m}^{A, A} \cdot m$ leaves are expanded by a copy of $\mathfrak{D}_{q, m}^{A, B}$. Another $m$ leaves are expanded by a copy of $\mathfrak{D}_{q, m}^{B, A}$.

$\left(2^{\exists}\right) \widetilde{\mathfrak{B}}_{m}^{p}$ is a point-expansion of $\mathcal{T}_{\exists, m}^{\mathfrak{B}}$ over $\mathcal{K}$ as follows: The root of $\mathcal{T}_{\exists, m}^{\mathfrak{B}}$ is expanded by $\mathcal{I}$. It is also called the "root" of $\widetilde{\mathfrak{B}}_{m}^{p}$ that interprets $r . m$ leaves are expanded by a copy of $\mathfrak{D}_{q, m}^{A, B}$. The other $m$ leaves are expanded by a copy of $\mathfrak{D}_{q, m}^{B, A}$. 
- If $p=\forall q$,

$\left(1^{\forall}\right)$ The lem of $\widetilde{\mathfrak{A}}_{m}^{p}$ is the same as the above lem $\left(2^{\exists}\right)$ of $\widetilde{\mathfrak{B}}_{m}^{p}$.

$\left(2^{\forall}\right)$ The lem of $\widetilde{\mathfrak{B}}_{m}^{p}$ is the same as the lem $\left(1^{\exists}\right)$ of $\widetilde{\mathfrak{A}}_{m}^{p}$ except that $\mathfrak{D}_{q, m}^{A, A}$ is replaced by a copy of $\mathfrak{D}_{q, m}^{B, B}$.

Remark 3.14. $\widetilde{\mathfrak{A}}_{m}^{p}$ is the same as $\widetilde{\mathfrak{B}}_{m}^{\bar{p}}$ except that the "colours" of leaves are flipped: black is interchanged with not black.

The structures $\widetilde{\mathfrak{A}}_{m}^{p}$ and $\widetilde{\mathfrak{B}}_{m}^{p}$ are trees with coloured edges and nodes. Note that only a leaf could be black by this definition because the root of a tree is not black by default. See Figure 1 for example, where $p=\exists \exists$ and $m=1$. Here, we use a solid line to represent a red edge and a dashed line to represent a blue edge.
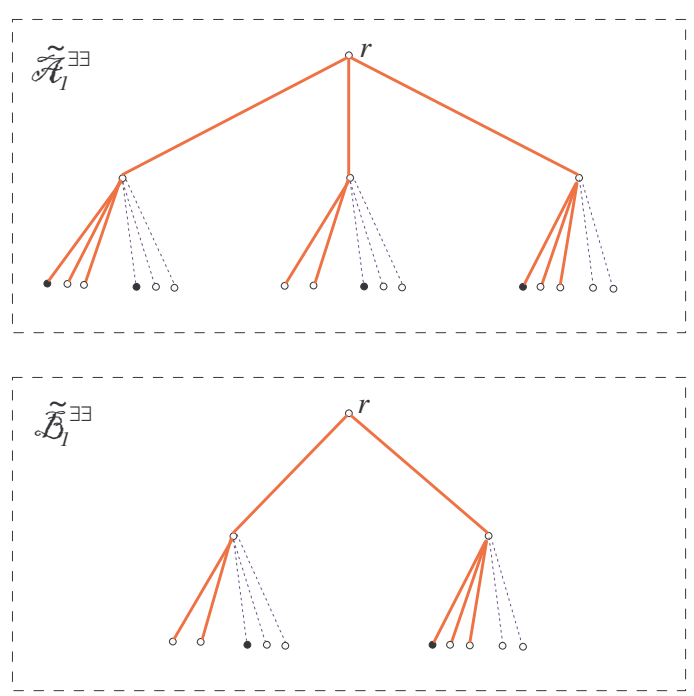

Figure 1: The structures $\widetilde{\mathfrak{A}}_{1}^{\exists}$ and $\widetilde{\mathfrak{B}}_{1}^{\exists}$.

We are going to define a formula $\widetilde{\varphi}_{p}$ for each string $p \in \Gamma^{*}$.

Definition 3.15. Let $q \in \Gamma^{*}$ and assume that $|q|=d \geq 0$.

(1) $\widetilde{\psi}_{\epsilon}(x)=\widetilde{\psi}_{-\epsilon}(x):=U(x)$;

(2) $\widetilde{\psi}_{\exists q}(y):=\exists x_{d+1}\left(R y x_{d+1} \wedge \widetilde{\psi}_{q}\left(x_{d+1}\right) \wedge \widetilde{\psi}_{-q}\left(x_{d+1}\right)\right)$;

$\widetilde{\psi}_{\forall q}(y):=\forall x_{d+1}\left(R y x_{d+1} \rightarrow \widetilde{\psi}_{q}\left(x_{d+1}\right) \vee \widetilde{\psi}_{-q}\left(x_{d+1}\right)\right) ;$

(3) $\widetilde{\psi}_{-\exists q}(y):=\exists x_{d+1}\left(B y x_{d+1} \wedge \widetilde{\psi}_{q}\left(x_{d+1}\right) \wedge \widetilde{\psi}_{-q}\left(x_{d+1}\right)\right)$;

$\widetilde{\psi}_{-\forall q}(y):=\forall x_{d+1}\left(B y x_{d+1} \rightarrow \widetilde{\psi}_{q}\left(x_{d+1}\right) \vee \widetilde{\psi}_{-q}\left(x_{d+1}\right)\right) ;$ 
Now we define a sentence over the signature $\tau^{+}$:

$$
\begin{aligned}
\widetilde{\varphi}_{p} & :=\widetilde{\psi}_{p}(r) ; \\
\widetilde{\varphi}_{-p} & :=\widetilde{\psi}_{-p}(r) .
\end{aligned}
$$

From now on, we assume that $m \in \mathbb{N}^{+}$is an arbitrary natural number except where defined explicitly in context (see Theorem 3.23 for example).

Lemma 3.16. $\widetilde{\mathfrak{A}}_{m}^{p} \models \widetilde{\varphi}_{p}$ iff $\widetilde{\mathfrak{A}}_{m}^{-p} \models \widetilde{\varphi}_{-p}$.

Proof. Even though $\widetilde{\mathfrak{A}}_{m}^{-p}$ is defined as a structure which is obtained from $\widetilde{\mathfrak{A}}_{m}^{p}$ by exchanging all the colours of the edges, we will prove that $\widetilde{\mathfrak{A}}_{m}^{p}$ can be turned into $\widetilde{\mathfrak{A}}_{m}^{-p}$ if we only exchange the colours of the edges that connect to root (from red to blue and from blue to red) in $\widetilde{\mathfrak{A}}_{m}^{p}$, and vice versa:

(1) The base cases when $|p|=1$ are trivial;

(2) Assume that it holds when $|p|=k$;

(3) Assume that $p=\exists q$ where $|q|=k$. According to the definition, the structure obtained from $\mathfrak{D}_{q, m}^{A, B}$ by exchanging the edge colours is $\mathfrak{D}_{q, m}^{B, A}$. Likewise, the structure obtained from $\mathfrak{D}_{q, m}^{B, A}$ by exchanging the edge colours is $\mathfrak{D}_{q, m}^{A, B}$. For the reason of symmetry, the structure obtained from $\mathfrak{D}_{q, m}^{A, A}$ by exchanging the edge colours is the same as $\mathfrak{D}_{q, m}^{A, A}$. Therefore, the conclusion holds according to the definition of $\widetilde{\mathfrak{A}}_{m}^{\exists q}$.

(4) Similarly, we can prove it holds when $p=\forall q$ where $|q|=k$. Therefore, it holds when $|p|=k+1$.

Also note that $\widetilde{\varphi}_{p}$ is the same as $\widetilde{\varphi}_{-p}$ except that $\widetilde{\varphi}_{p}$ claims that the edges that connect to root are red and $\widetilde{\varphi}_{-p}$ claims that the edges that connect to root are blue. Therefore, $\widetilde{\mathfrak{A}}_{m}^{p} \models \widetilde{\varphi}_{p}$ iff $\widetilde{\mathfrak{A}}_{m}^{-p} \models \widetilde{\varphi}_{-p}$.

Lemma 3.17. $\widetilde{\mathfrak{A}}_{m}^{p} \models \widetilde{\varphi}_{p}$ and $\widetilde{\mathfrak{B}}_{m}^{p} \not \models \widetilde{\varphi}_{p}$.

Proof. It is obvious when $p=\exists$ or $\forall$. Assume that it holds when $|p|=k$.

If $p=\exists q, \widetilde{\mathfrak{A}}_{m}^{p}$ is composed of a node $a_{r}$, a copy of $\mathfrak{D}_{q, m}^{A, A}, m$ copies of $\mathfrak{D}_{q, m}^{A, B}$ and $m$ copies of $\mathfrak{D}_{q, m}^{B, A} ; \widetilde{\varphi}_{p}=\exists x_{k+1}\left(\operatorname{Rr} x_{k+1} \wedge \widetilde{\psi}_{q}\left(x_{k+1}\right) \wedge \widetilde{\psi}_{-q}\left(x_{k+1}\right)\right)$. Let $a_{r}$ interpret $r$ in $\widetilde{\mathfrak{A}}_{m}^{p}$, and the junction point $b_{r}$ of $\mathfrak{D}_{q, m}^{A, A}$ witness $\exists x_{k+1}$ in $\widetilde{\varphi}_{p}$. Note that $b_{r}$ divides $\mathfrak{D}_{q, m}^{A, A}$ into two parts: one is an isomorphic copy of $\widetilde{\mathfrak{A}}_{m}^{q}$ and the other is an isomorphic copy of $\widetilde{\mathfrak{A}}_{m}^{-q}$. For convenience, we still use $\widetilde{\mathfrak{A}}_{m}^{q}$ and $\widetilde{\mathfrak{A}}_{m}^{-q}$ to denote these copies. Therefore, when $r$ is interpreted as $b_{r}$, $\widetilde{\mathfrak{A}}_{m}^{q}=\widetilde{\psi}_{q}(r)$ and $\widetilde{\mathfrak{A}}_{m}^{-q}=\widetilde{\psi}_{-q}(r)$ (according to the induction hypothesis and Lemma 3.16.

Moreover, all the quantifiers in $\widetilde{\psi}_{q}$ are relativized by relations either $R y x$ or $B y x$, where $x$ is the quantified variable. And $\widetilde{\psi}_{q}$ expresses some property that has nothing to do with the elements outside the tree substructure $\widetilde{\mathfrak{A}}_{m}^{q}$. More precisely, in Definition 3.15 the variable " $y$ " does not occur free in the formulas $\widetilde{\psi}_{q}\left(x_{d+1}\right)$ and $\widetilde{\psi}_{-q}\left(x_{d+1}\right)$. As a consequence, $\widetilde{\mathfrak{A}}_{m}^{q}=\widetilde{\psi}_{q}(r)$ implies $\widetilde{\mathfrak{A}}_{m}^{p} \models \widetilde{\psi}_{q}\left(b_{r}\right)$.

By the same argument, $\widetilde{\mathfrak{A}}_{m}^{-q}=\widetilde{\psi}_{-q}(r)$ implies $\widetilde{\mathfrak{A}}_{m}^{p} \models \widetilde{\psi}_{-q}\left(b_{r}\right)$. Therefore, $\widetilde{\mathfrak{A}}_{m}^{p} \models \widetilde{\varphi}_{p}$.

Let $c_{r}$ be a child of $r^{\widetilde{\mathfrak{B}}_{m}^{p}}$ in $\widetilde{\mathfrak{B}}_{m}^{p}$. By assumption, $\widetilde{\mathfrak{B}}_{m}^{q} \not \neq \widetilde{\psi}_{q}(r)\left(\widetilde{\mathfrak{B}}_{m}^{-q} \not \models \widetilde{\psi}_{-q}(r)\right.$ resp. $)$ where $c_{r}$ interprets $r$ in $\widetilde{\mathfrak{B}}_{m}^{q}\left(\widetilde{\mathfrak{B}}_{m}^{-q}\right.$ resp.). As explained before, this means $\widetilde{\mathfrak{B}}_{m}^{p} \not \widetilde{\psi}_{q}\left(c_{r}\right)$ $\left(\widetilde{\mathfrak{B}}_{m}^{p} \not \models \widetilde{\psi}_{-q}\left(c_{r}\right)\right.$ resp.). So, $c_{r}$ cannot be a witness of $\exists x_{k+1}$. Therefore, $\widetilde{\mathfrak{B}}_{m}^{p} \not \neq \widetilde{\varphi}_{p}$. 
Similarly, we can prove that it also holds when $p=\forall q$. Hence, it holds when $|p|=k+1$.

Let $S$ and $S^{\prime}$ be two finite $\Gamma$-labelled forests. We collect some simple facts below.

Lemma 3.18. $\widetilde{\mathfrak{A}}_{m}^{p} \rightsquigarrow_{S} \widetilde{\mathfrak{B}}_{m}^{p}$ iff $\widetilde{\mathfrak{A}}_{m}^{-p} \rightsquigarrow_{S} \widetilde{\mathfrak{B}}_{m}^{-p}$.

Proof. According to the definitions, $\widetilde{\mathfrak{A}}_{m}^{-p}\left(\widetilde{\mathfrak{B}}_{m}^{-p}\right.$ resp.) is similar to $\widetilde{\mathfrak{A}}_{m}^{p}\left(\widetilde{\mathfrak{B}}_{m}^{p}\right.$ resp.) except that the colours of their edges are exchanged. Therefore, the duplicator can mimic her winning strategy in one game when she plays in the other.

Definition 3.19. Let $\mathfrak{A} \bigoplus_{a} \mathfrak{B}$, a structure over signature $\sigma$, be the join of two disjoint $\sigma$-substructures $\mathfrak{A}$ and $\mathfrak{B}$ at its element $a$. That is,

(1) $|\mathfrak{A}| \cap|\mathfrak{B}|=\{a\}$;

(2) $\left|\mathfrak{A} \bigoplus_{\mathfrak{a}} \mathfrak{B}\right|=|\mathfrak{A}| \cup|\mathfrak{B}|$.

(3) For any $R \in \sigma, R^{\mathfrak{A} \oplus_{a} \mathfrak{B}}:=R^{\mathfrak{A}} \cup R^{\mathfrak{B}}$.

Lemma 3.20. Let $S$ be a $\Gamma$-labelled forest. $\mathfrak{A} \bigoplus_{a} \mathfrak{B} \rightsquigarrow_{S} \mathfrak{A} \bigoplus_{b} \mathfrak{B}^{\prime}$ if there is an automorphism $h$ of $\mathfrak{A}$ s.t. $b=h(a)$ and $\mathfrak{B} \rightsquigarrow S \mathfrak{B}^{\prime}$.

Proof. Assume that both $\mathfrak{B}$ and $\mathfrak{B}^{\prime}$ are $\sigma$-structures. Let $\sigma^{+}:=\sigma \cup\left\{c_{B}\right\}$ where $c_{B}$ is called a hook. Let $\check{\mathfrak{B}}\left(\check{\mathfrak{B}^{\prime}}\right.$ resp.) be an expansion of $\mathfrak{B}\left(\mathfrak{B}^{\prime}\right.$ resp.) to $\sigma^{+}$. Let $\mathcal{K}=\{\mathcal{I}, \check{\mathfrak{B}}\}$ and $\mathcal{E}_{\mathcal{K}}^{J}(\mathfrak{A})$ be a point-expansion of $\mathfrak{A}$ over $\mathcal{K}$ defined by $\mathbb{I}$ such that the element $a$ of $\mathfrak{A}$ is expanded by $\check{\mathfrak{B}}$ and all the other elements are expanded by $\mathcal{I}$. Similarly, let $\mathcal{K}^{\prime}=\{\mathcal{I}, \check{\mathfrak{B}}\}$ and $\mathcal{E}_{\mathcal{K}^{\prime}}^{\beth^{\prime}}(\mathfrak{A})$ be a point-expansion of $\mathfrak{A}$ over $\mathcal{K}^{\prime}$ defined by $\beth^{\prime}$ such that the element $b$ of $\mathfrak{A}$ is expanded by $\check{\mathfrak{B}}^{\prime}$ and all the other elements are expanded by $\mathcal{I}$. If there is an automorphism $h$ of $\mathfrak{A}$ s.t. $b=h(a)$, then by Lemma 3.11 the following holds:

$$
\mathcal{E}_{\mathcal{K}}^{\beth}(\mathfrak{A}) \rightsquigarrow_{S} \mathcal{E}_{\mathcal{K}^{\prime}}^{\beth^{\prime}}(\mathfrak{A}) \text { if } \mathfrak{B} \rightsquigarrow_{S} \mathfrak{B}^{\prime} .
$$

Observe that $\mathcal{E}_{\mathcal{K}}^{\mathcal{J}}(\mathfrak{A})$ is exactly $\mathfrak{A} \bigoplus_{a} \mathfrak{B}$ and $\mathcal{E}_{\mathcal{K}^{\prime}}^{\mathcal{J}^{\prime}}(\mathfrak{A})$ is exactly $\mathfrak{A} \bigoplus_{b} \mathfrak{B}^{\prime}$. Hence, the lemma holds.

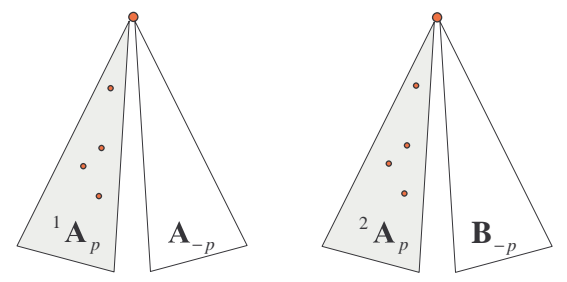

Figure 2: $\left(\mathfrak{D}_{p, m}^{A, A}, \bar{a}\right)$ and $\left(\mathfrak{D}_{p, m}^{A, B}, \bar{b}\right)$ where $\bar{a}$ includes $r^{1 \widetilde{\mathfrak{A}}_{m}^{p}}$ and $\bar{b}$ includes $r^{2 \widetilde{\mathfrak{A}}_{m}^{p}}$.

We use ${ }^{1} \widetilde{\mathfrak{A}}_{m}^{p}$ and ${ }^{2} \widetilde{\mathfrak{A}}_{m}^{p}$ to denote two isomorphic copies of $\widetilde{\mathfrak{A}}_{m}^{p}$ in $\mathfrak{D}_{p, m}^{A, A}$ and $\mathfrak{D}_{p, m}^{A, B}$ respectively (see Figure 2. Note that in the picture we use " ${ }^{1} \mathrm{~A}_{p}$ " to denote ${ }^{1} \widetilde{\mathfrak{A}}_{m}^{p}$.). The superscripts " 1 " and " 2 " are used to distinguish these two copies. Let $\bar{a} \in\left|{ }^{1} \widetilde{\mathfrak{A}}_{m}^{p}\right|^{k}$ and $\bar{b} \in\left|{ }^{2} \widetilde{\mathfrak{A}}_{m}^{p}\right|^{k}$. The following lemma is a special case of Lemma 3.20 . 
Lemma 3.21. Assume that $\left({ }^{1} \widetilde{\mathfrak{A}}_{m}^{p}, \bar{a}\right) \cong\left({ }^{2} \widetilde{\mathfrak{A}}_{m}^{p}, \bar{b}\right)$. Then,

$$
\left(\mathfrak{D}_{p, m}^{A, A}, \bar{a}\right) \rightsquigarrow S\left(\mathfrak{D}_{p, m}^{A, B}, \bar{b}\right) \text { if } \widetilde{\mathfrak{A}}_{m}^{-p} \rightsquigarrow S \widetilde{\mathfrak{B}}_{m}^{-p}
$$

Clearly, for two $\Gamma$-labelled forests $S_{1}$ and $S_{2}, \mathscr{W}\left(S_{1}\right) \subseteq \mathscr{W}\left(S_{2}\right)$ if $S_{1} \preceq{ }_{e} S_{2}$. Let $f_{m}^{p}:=\{q \in$ $\left.\gamma^{-}(f(p))|| q \mid \leq m\right\}$.

Lemma 3.22. Let $p \in \Gamma^{*}$ and $m \in \mathbb{N}^{+}$, then the following holds:

(1) The duplicator has a winning strategy in $G_{\mathscr{F}\left(f_{m}^{p}\right)}\left(\widetilde{\mathfrak{A}}_{m}^{p}, \widetilde{\mathfrak{B}}_{m}^{p}\right)$;

(2) For any $\psi$ such that $\mathscr{W}(q s(\psi)) \subseteq f_{m}^{p}$, we have that

$$
\widetilde{\mathfrak{A}}_{m}^{p} \models \psi \Rightarrow \widetilde{\mathfrak{B}}_{m}^{p} \models \psi \text {. }
$$

Proof. By Lemma 2.13, $q s(\psi) \preceq_{e} \mathscr{F}\left(f_{m}^{p}\right)$. Hence by Theorem 3.8 and Lemma 3.6, (2) is implied by (1) and we need only prove (1).

Note that $\widetilde{\varphi}_{p}$ is obtained from $\neg \widetilde{\varphi}_{\bar{p}}$ by adding a " $\neg$ " before all the occurrences of the unary predicate $U$. And $\widetilde{\varphi}_{p}$ is equivalent to a sentence in $\operatorname{FO}\{f(p)\}$ iff $\neg \widetilde{\varphi}_{\bar{p}}$ is equivalent to a sentence in $\mathrm{FO}\{f(p)\}$ iff $\widetilde{\varphi}_{\bar{p}}$ is equivalent to a sentence in $\operatorname{FO}\{f(\bar{p})\}$ (the second "iff" is due to Lemma 2.3. That is, the duplicator has a winning strategy in $G_{\mathscr{F}\left(f_{m}^{p}\right)}\left(\widetilde{\mathfrak{A}}_{m}^{p}, \widetilde{\mathfrak{B}}_{m}^{p}\right)$ iff she has a winning strategy in $G_{\mathscr{F}\left(f_{m}^{\bar{p}}\right)}\left(\widetilde{\mathfrak{A}}_{m}^{\bar{p}}, \widetilde{\mathfrak{B}}_{m}^{\bar{p}}\right)$. Therefore, we need only consider the case when $p[1]=\exists$ since the lemma holds when $p[1]=\exists$ iff it holds when $p[1]=\forall$. Soon we shall see that the case when $p[2]=\forall$ is different from the case when $p[2]=\exists$, provided that $p[1]=\exists$. Therefore, we discuss them separately.

Let $|p|=d$. When $d=1$ i.e. $p=\exists, f(p)=\forall^{*}$. In the game, the spoiler can only pick at most $m$ distinct elements in $\widetilde{\mathfrak{B}}_{m}^{p}$. And $\widetilde{\mathfrak{A}}_{m}^{p}$ has $2 m$ distinct elements that are not black. Hence, the duplicator is able to mimic the spoiler's picking as follows. She picks the root of $\widetilde{\mathfrak{A}}_{m}^{p}$ if the spoiler picks the root of $\widetilde{\mathfrak{B}}_{m}^{p}$; she picks a leaf that is not black in $\widetilde{\mathfrak{A}}_{m}^{p}$ if the spoiler picks a leaf of $\widetilde{\mathfrak{B}}_{m}^{p}$. That is, the duplicator has a winning strategy in this game. Similarly, when $p=\forall$, the duplicator also win the game.

Assume that it holds when $d \leq k$ for some $k \geq 1$. That is, the duplicator has a winning strategy in the game $G_{\mathscr{F}\left(f_{m}^{p}\right)}\left(\widetilde{\mathfrak{A}}_{m}^{p}, \widetilde{\mathfrak{B}}_{m}^{p}\right)$ for any $|p| \leq k$ and any $m \in \mathbb{N}^{+}$.

Assume that $p=\exists \forall q$ where $|q|=k-1$. Then $f(p)=\forall^{*} * f(\forall q)$. This case is relatively easy to explain.

The strategy of the duplicator in the game $G_{\mathscr{F}\left(f_{m}^{\forall q}\right)}\left(\mathcal{T}_{\exists, m}^{\mathfrak{A}}, \mathcal{T}_{\exists, m}^{\mathfrak{B}}\right)$ is very simple:

(I) If the spoiler picks the junction point of $\mathfrak{D}_{\forall q, m}^{A, A}$ that is a leaf of $\mathcal{T}_{\exists, m}^{\mathfrak{A}}$, the duplicator replies with a junction point of one copy of $\mathfrak{D}_{\forall q, m}^{A, B}$, called $\mathfrak{D}_{A}$, that is a leaf of $\mathcal{T}_{\exists, m}^{\mathfrak{B}}$.

(II) If the spoiler picks a junction point of $\mathfrak{D}_{\forall q, m}^{A, B}$ or $\mathfrak{D}_{\forall q, m}^{B, A}$ that is a leaf of one tree and that is not $\mathfrak{D}_{A}$, the duplicator replies with a junction point of an isomorphic copy that is a leaf of the other tree.

(III) If the spoiler picks an element, say $a$, which has been picked before, the duplicator picks $b$, which was picked in the same round when $a$ was picked.

We can regard the words that can be read off $\mathscr{F}\left(f_{m}^{p}\right)$ as the "resource" that the spoiler can use to detect the difference between the structures. Note that the first universal quantifier block in the words that can possibly be read off $\mathscr{F}\left(f_{m}^{p}\right)$ is useless for the spoiler: no matter how he picks in $\widetilde{\mathfrak{B}}_{m}^{p}$, the duplicator always picks an isomorphic substructure and mimics the spoiler's picks in the isomorphic substructure. By Lemma 3.20, if the spoiler can win in the end, he can also win if the players do not pick in these isomorphic substructures. 
Let $Q:=\left\{s \in f_{j}^{\forall q} \mid 0 \leq j \leq m\right\}$. Clearly, $Q \preceq f_{m}^{\forall q}$.

Observe that the strategy described before is a winning strategy for the duplicator in the game $G_{\mathscr{F}\left(f_{m}^{\forall q}\right)}\left(\mathcal{T}_{\exists, m}^{\mathfrak{A}}, \mathcal{T}_{\exists, m}^{\mathfrak{B}}\right)$. By induction hypothesis, she also has a winning strategy in the game $G_{\mathscr{F}\left(f_{m}^{\forall q}\right)}\left(\widetilde{\mathfrak{A}}_{m}^{\forall q}, \widetilde{\mathfrak{B}}_{m}^{\forall q}\right)$. Hence, by Lemma 3.21 and Lemma 3.18, $\mathfrak{D}_{\forall q, m}^{A, A} \rightsquigarrow f_{m}^{\forall q} \mathfrak{D}_{\forall q, m}^{A, B}$. Moreover, the winning strategies of the duplicator in the games $G_{\mathscr{F}\left(f_{m}^{\forall q}\right)}\left(\mathcal{T}_{\exists, m}^{\mathfrak{A}}, \mathcal{T}_{\exists, m}^{\mathfrak{B}}\right)$ and $G_{\mathscr{F}\left(f_{m}^{\forall q}\right)}\left(\mathfrak{D}_{\forall q, m}^{A, A}, \mathfrak{D}_{\forall q, m}^{A, B}\right)$ can be combined together: if the spoiler picks a leaf of $\mathcal{T}_{\exists, m}^{\mathfrak{B}}\left(\mathcal{T}_{\exists, m}^{\mathfrak{A}}\right.$ resp.) that is the junction point of $\mathfrak{D}_{\forall q, m}^{A, B}$ or $\mathfrak{D}_{\forall q, m}^{B, A}$ (not $\mathfrak{D}_{A}$ ), the duplicator picks a leaf of $\mathcal{T}_{\exists, m}^{\mathfrak{A}}\left(\mathcal{T}_{\exists, m}^{\mathfrak{B}}\right.$ resp. $)$ that is the junction point of an isomorphic structure; if the spoiler picks a leaf of $\mathcal{T}_{\exists, m}^{\mathfrak{A}}$ that is the junction point of $\mathfrak{D}_{\forall q, m}^{A, A}$, the duplicator picks a leaf of $\mathcal{T}_{\exists, m}^{\mathfrak{B}}$ that is the junction point of $\mathfrak{D}_{A}$. Therefore, she has a combined winning strategy in the game $G_{\mathscr{F}\left(f_{m}^{\forall q}\right)}\left(\widetilde{\mathfrak{A}}_{m}^{p}, \widetilde{\mathfrak{B}}_{m}^{p}\right)$, by Lemma 3.11. By Lemma 3.6, she also has a winning strategy in the game $G_{\mathscr{F}(Q)}\left(\widetilde{\mathfrak{A}}_{m}^{p}, \widetilde{\mathfrak{B}}_{m}^{p}\right)$. Note that $f_{m}^{p}=\left\{s \in \Gamma^{m} \mid s=\forall^{i} * s^{\prime}\right.$ where $i \leq m$ and $\left.s^{\prime} \in Q\right\}$. In other words, if we remove the path initiating from a root in $\mathscr{F}\left(f_{m}^{p}\right)$ where the word that can be read off the path is $\forall^{m}$, we can turn $\mathscr{F}\left(f_{m}^{p}\right)$ into $\mathscr{F}(Q)$. Recall that the first universal quantifier block in the words that can be read off $\mathscr{F}\left(f_{m}^{p}\right)$ is useless for the spoiler. Therefore, the duplicator has a winning strategy in the game $G_{\mathscr{F}\left(f_{m}^{p}\right)}\left(\widetilde{\mathfrak{A}}_{m}^{p}, \widetilde{\mathfrak{B}}_{m}^{p}\right)$.

Assume that $p=\exists \exists q$ where $|q|=k-1$ (see Figure 3). Then $f(p)=\forall^{*} \exists * f(\exists q)$. As we have explained before, the first universal quantifier block in the words that can possibly be read off $\mathscr{F}\left(f_{m}^{p}\right)$ is useless for the spoiler.

The strategy of the duplicator in the game $G_{\mathscr{F}\left(f_{m}^{\exists q}\right)}\left(\mathcal{T}_{\exists, m}^{\mathfrak{A}}, \mathcal{T}_{\exists, m}^{\mathfrak{B}}\right)$ is the same as her strategy in the game $G_{\mathscr{F}\left(f_{m}^{\forall q}\right)}\left(\mathcal{T}_{\exists, m}^{\mathfrak{A}}, \mathcal{T}_{\exists, m}^{\mathfrak{B}}\right)$.

For the first existential quantifier that can possibly be read off $\mathscr{F}\left(f_{m}^{p}\right)$, there are several choices for the spoiler:

- Picking the root (or picking a junction point resp.).

The game is reduced to a composition of the main game $G_{\mathscr{F}\left(f_{m}^{\exists q}\right)}\left(\mathcal{T}_{\exists, m}^{\mathfrak{A}}, \mathcal{T}_{\exists, m}^{\mathfrak{B}}\right)$ and the subgames in which the duplicator has a winning strategy, that is, in the subgames

$$
G_{\mathscr{F}\left(f_{m}^{\exists q}\right)}\left(\mathfrak{D}_{\exists q, m}^{A, A}, \mathfrak{D}_{\exists q, m}^{A, B}\right) \text { or } G_{\mathscr{F}\left(f_{m}^{\exists q}\right)}\left(\left(\mathfrak{D}_{\exists q, m}^{A, A}, r^{1 \widetilde{\mathfrak{A}}_{m}^{p}}\right),\left(\mathfrak{D}_{\exists q, m}^{A, B}, r^{2 \widetilde{\mathfrak{A}}_{m}^{p}}\right)\right),
$$

or subgames between isomorphic structures, according to Lemma 3.18 and Lemma 3.21 . And by Lemma 3.11, the duplicator has a winning strategy.

- Picking inside the structure, either $\mathfrak{D}_{\exists q, m}^{A, A}$ or $\mathfrak{D}_{\exists q, m}^{A, B}$ or $\mathfrak{D}_{\exists q, m}^{B, A}$, except their junction points. When the spoiler picks inside $\mathfrak{D}_{\exists q, m}^{A, B}$ or $\mathfrak{D}_{\exists q, m}^{B, A}$, the duplicator can mimic it in an isomorphic copy. Hence, only one new case need be taken care of: the spoiler picks the element inside $\mathfrak{D}_{\exists q, m}^{A, A}$. In this case, the duplicator has a strategy as follows:

(i) If the spoiler picks the element inside $\widetilde{\mathfrak{A}}_{m}^{\exists q}$, the duplicator mimics his picking in the isomorphic copy that is a part of $\mathfrak{D}_{\exists q, m}^{A, B}$.

(ii) If the spoiler picks the element inside $\widetilde{\mathfrak{A}}_{m}^{-\exists q}$, the duplicator mimics his picking in the isomorphic copy that is a part of $\mathfrak{D}_{\exists q, m}^{B, A}$.

According to Lemma 3.20, picking in these isomorphic substructures doesn't influence the outcome. In either case, the game is reduced to a composition of the main game $G_{\mathscr{F}\left(f_{m}^{\exists q}\right)}\left(\mathcal{T}_{\exists, m}^{\mathfrak{A}}, \mathcal{T}_{\exists, m}^{\mathfrak{B}}\right)$ and the subgame $G_{\mathscr{F}\left(f_{m}^{\exists q}\right)}\left(\widetilde{\mathfrak{A}}_{m}^{\exists q}, \widetilde{\mathfrak{B}}_{m}^{\exists q}\right)$ or $G_{\mathscr{F}\left(f_{m}^{\exists q}\right)}\left(\widetilde{\mathfrak{A}}_{m}^{-\exists q}, \widetilde{\mathfrak{B}}_{m}^{-\exists q}\right)$ or a 

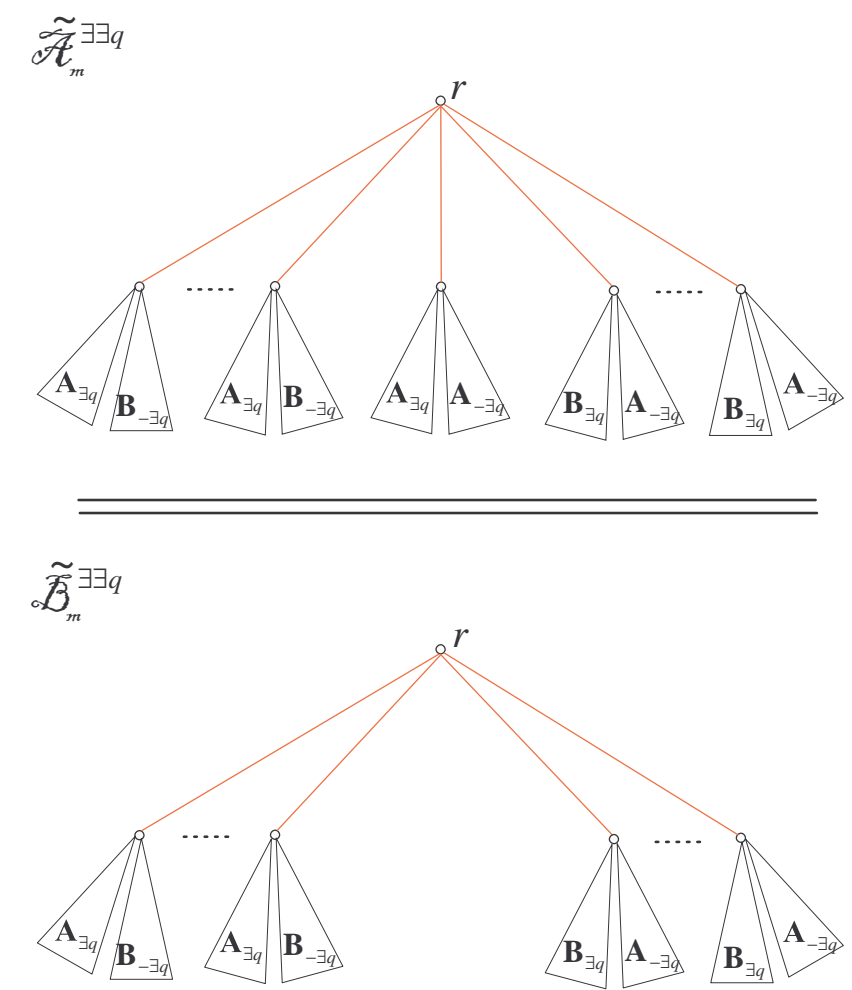

Figure 3: The structures $\widetilde{\mathfrak{A}}_{m}^{\exists \exists q}$ and $\widetilde{\mathfrak{B}}_{m}^{\exists \exists q}$.

subgame between two isomorphic structures. By the assumption we know that the duplicator has a winning strategy in $G_{\mathscr{F}\left(f_{m}^{\exists q}\right)}\left(\widetilde{\mathfrak{A}}_{m}^{\exists q}, \widetilde{\mathfrak{B}}_{m}^{\exists q}\right)$. Furthermore, by Lemma 3.18, the duplicator has a winning strategy in $G_{\mathscr{F}\left(f_{m}^{\exists q}\right)}\left(\widetilde{\mathfrak{A}}_{m}^{-\exists q}, \widetilde{\mathfrak{B}}_{m}^{-\exists q}\right)$. Finally, by Lemma 3.21 , Lemma 3.6 and Lemma 3.11 , the duplicator has a winning strategy in $G_{\mathscr{F}\left(f_{m}^{p}\right)}\left(\widetilde{\mathfrak{A}}_{m}^{p}, \widetilde{\mathfrak{B}}_{m}^{p}\right)$. When $p[1]=\exists$, we have proved that $\widetilde{\mathfrak{A}}_{m}^{p} \rightsquigarrow f_{m}^{p} \widetilde{\mathfrak{B}}_{m}^{p}$. Therefore, this theorem follows when $p[1]=\exists$. As a consequence it also holds when $p[1]=\forall$ by previous analysis.

Theorem 3.23. Let $S_{1}$ and $S_{2}$ be two finite $\Gamma$-labelled forests. Over the class of all finite $\tau^{+}$-structures,

$$
\text { if } \mathscr{W}\left(S_{1}\right) \nsubseteq \mathscr{W}\left(S_{2}\right) \text {, then } \mathrm{FO}\left\{S_{1}\right\} \nsubseteq \mathrm{FO}\left\{S_{2}\right\}
$$

Proof. Let $p \in \mathscr{W}\left(S_{1}\right) \backslash \mathscr{W}\left(S_{2}\right)$. According to Lemma 2.4. $\gamma^{-}(f(p))$ is the set of all prefixes that $p$ is not a subsequence of. Hence, $\mathscr{W}\left(S_{2}\right) \subseteq \gamma^{-}(f(p))$. Clearly, $\widetilde{\varphi}_{p} \in \mathrm{FO}\left\{S_{1}\right\}$. By Lemma 3.17, for any positive natural number $n, \widetilde{\mathfrak{A}}_{n}^{p} \models \widetilde{\varphi}_{p}$ but $\widetilde{\mathfrak{B}}_{n}^{p} \not \models \widetilde{\varphi}_{p}$. Assume for the purpose of a contradiction that there is a formula $\psi$ such that $q s(\psi) \preceq_{e} S_{2}, q r(\psi)=m$ and $\psi$ defines the same property as $\widetilde{\varphi}_{p}$. Clearly, $\mathscr{W}(q s(\psi)) \preceq f_{m}^{p}$. By Lemma $3.22, \widetilde{\mathfrak{A}}_{m}^{p}=\psi \Rightarrow \widetilde{\mathfrak{B}}_{m}^{p} \models \psi$. 
Together with Lemma 3.17, and Corollary 3.9, the property defined by $\widetilde{\varphi}_{p}$ is not definable by any first-order sentence whose quantifier structure is $\mathcal{F}$ s.t. $\mathscr{W}(\mathcal{F}) \subseteq f_{m}^{p}$, which is in contradiction with the assumption that $\psi$ defines the same property as $\widetilde{\varphi}_{p}$ does.

If we want to prove something similar to Theorem 3.23 , but over a restricted signature $\langle r, E\rangle$, then we need to adapt the constructions and formulas a bit. Note that we can use forward arrows and backward arrows to replace the red edges and blue edges. And we can use bi-directional edges to indicate where the black leaves are. More precisely, the new structure $\mathfrak{A}_{m}^{p \prime}{ }^{\prime}\left(\mathfrak{B}_{m}^{p \prime}\right.$ resp.) is obtained from $\widetilde{\mathfrak{A}}_{m}^{p}\left(\widetilde{\mathfrak{B}}_{m}^{p}\right.$ resp.) by the following process:

(1) Use an arc from $b$ to $c$ to represent that the edge between the vertices $b$ and $c$ is red;

(2) Use an arc from $c$ to $b$ to represent that the edge between $b$ and $c$ is blue;

(3) Add an edge from every black leaf to the junction point in the same connected component and from the junction point to every black leaf in the same connected component;

(4) When $|p|=2$, add a self loop to every leaf which is an endpoint of a red edge.

Correspondingly, the new formula, called $\varphi_{p}^{\prime}$, is obtained from $\widetilde{\varphi}_{p}$ by the following process:

$\left(1^{*}\right) R x y$ is replaced by Exy;

$\left(2^{*}\right) B x y$ is replaced by $E y x$;

$\left(3^{*}\right)$ When $|p| \neq 2, U y$ is replaced by $E x_{1} y \wedge E y x_{1}$;

$\left(4^{*}\right)$ When $|p|=2, U x_{1}$ in $\widetilde{\psi}_{\exists}(y)$ and $\widetilde{\psi}_{\forall}(y)$ is replaced by $E x_{1} y \wedge E y x_{1} \wedge E x_{1} x_{1} ; U x_{1}$ in $\widetilde{\psi}_{-\exists}(y)$ and $\widetilde{\psi}_{-\forall}(y)$ is replaced by $E x_{1} y \wedge E y x_{1} \wedge \neg E x_{1} x_{1}$.

More precisely, we inductively define $\varphi_{p}^{\prime}$ as follows. Let $q \in \Gamma^{*}$ and assume $|q|=d \geq 0$.

(1) $\psi_{\epsilon}^{\prime}(x, y)=\psi_{-\epsilon}^{\prime}(x, y):=E x y \wedge E y x$

(2) $\psi_{\exists q}^{\prime}(x, y):=\exists x_{d+1}\left(E y x_{d+1} \wedge x_{d+1} \neq x \wedge \psi_{q}^{\prime}\left(y, x_{d+1}\right) \wedge \psi_{-q}^{\prime}\left(y, x_{d+1}\right)\right)$;

$\psi_{\forall q}^{\prime}(x, y):=\forall x_{d+1}\left(E y x_{d+1} \wedge x_{d+1} \neq x \rightarrow \psi_{q}^{\prime}\left(y, x_{d+1}\right) \vee \psi_{-q}^{\prime}\left(y, x_{d+1}\right)\right) ;$

(3) $\psi_{-\exists q}^{\prime}(x, y):=\exists x_{d+1}\left(E x_{d+1} y \wedge x_{d+1} \neq x \wedge \psi_{q}^{\prime}\left(y, x_{d+1}\right) \wedge \psi_{-q}^{\prime}\left(y, x_{d+1}\right)\right)$;

$\psi_{-\forall q}^{\prime}(x, y):=\forall x_{d+1}\left(E x_{d+1} y \wedge x_{d+1} \neq x \rightarrow \psi_{q}^{\prime}\left(y, x_{d+1}\right) \vee \psi_{-q}^{\prime}\left(y, x_{d+1}\right)\right) ;$

(4) Now we define a sentence $\varphi_{p}^{\prime}$ over the signature $\tau$ :

- When $|p| \neq 2$,

$\varphi_{p}^{\prime}:=\psi_{p}^{\prime}(r, r)$

(note that $\varphi_{-p}^{\prime}:=\psi_{-p}^{\prime}(r, r)$ ).

- When $|p|=2$,

$$
\begin{aligned}
& \varphi_{\exists \exists}^{\prime}:=\exists x_{2}\left(E x_{2} x_{2} \wedge \exists x_{1}\left(E x_{2} x_{1} \wedge E x_{1} x_{2} \wedge E x_{1} x_{1}\right) \wedge \exists x_{1}\left(E x_{1} x_{2} \wedge E x_{2} x_{1} \wedge \neg E x_{1} x_{1}\right)\right) \\
& \varphi_{\exists \forall}^{\prime}:=\exists x_{2}\left(E x_{2} x_{2} \wedge \forall x_{1}\left(E x_{2} x_{1} \rightarrow E x_{1} x_{2} \wedge E x_{1} x_{1}\right)\right. \\
& \left.\wedge \forall x_{1}\left(E x_{1} x_{2} \rightarrow E x_{2} x_{1} \wedge \neg E x_{1} x_{1}\right)\right) \\
& \varphi_{\forall \exists}^{\prime}:=\forall x_{2}\left(E x_{2} x_{2} \rightarrow \exists x_{1}\left(E x_{2} x_{1} \wedge E x_{1} x_{2} \wedge E x_{1} x_{1}\right) \vee \exists x_{1}\left(E x_{1} x_{2} \wedge E x_{2} x_{1} \wedge \neg E x_{1} x_{1}\right)\right) \\
& \varphi_{\forall \forall}^{\prime}:=\forall x_{2}\left(E x_{2} x_{2} \rightarrow \forall x_{1}\left(E x_{2} x_{1} \rightarrow E x_{1} x_{2} \wedge E x_{1} x_{1}\right)\right. \\
& \left.\vee \forall x_{1}\left(E x_{1} x_{2} \rightarrow E x_{2} x_{1} \wedge \neg E x_{1} x_{1}\right)\right) \text {. }
\end{aligned}
$$

Let $\tau:=\langle E\rangle$ where $E$ is a binary relation symbol. Could we prove something similar to Theorem 3.23 , but over $\tau$ ? To achieve it, in addition to the adaption we have introduced above, we need to find a way to get rid of the root, which is obvious. The $\tau$-structure $\mathfrak{A}_{m}^{p}$ is obtained from $\mathfrak{A}_{m}^{p \prime}$ by the following process: removing $r^{\mathfrak{A}_{m}^{p}{ }^{\prime}}$ and for any junction point $v$, using a self loop at node $v$ to replace the edge from $r^{\mathfrak{A}_{m}^{p}{ }^{\prime}}$ to $v$.

$\mathfrak{B}_{m}^{p}$ is obtained from $\mathfrak{B}_{m}^{p \prime}$ in exactly the same way. 
Correspondingly, $\varphi_{p}$ is obtained from $\varphi_{p}^{\prime}$ by substituting $\operatorname{Rr} x$ with Exx and removing the atoms $x \neq r$.

Remark 3.24. It is a special case when $|p|=2$ because using bi-directional edges as a scheme of colouring does not work in this case. See Figures 4 for example, when $p=\exists \exists$ and $m=1$.
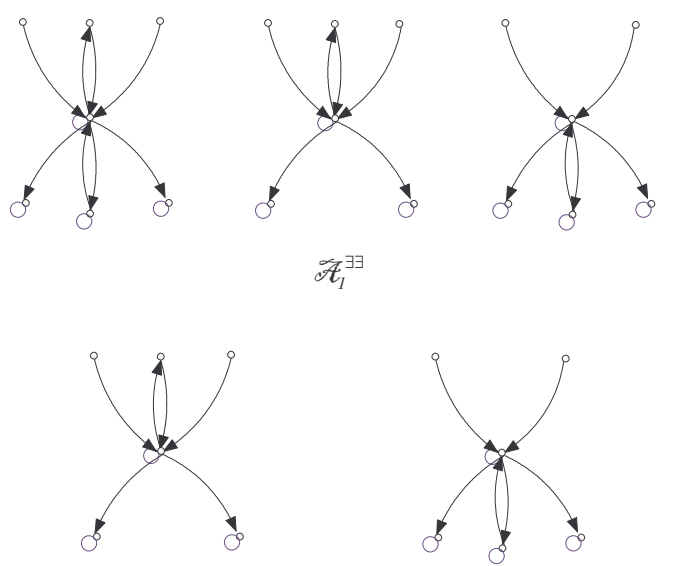

$\mathscr{\mathcal { B }}_{1}^{\exists \exists}$

Figure 4: The structures $\mathfrak{A}_{1}^{\exists}$ and $\mathfrak{B}_{1}^{\exists}$.

Call the above reductions between structures and formulas "reductions from $\tau^{+}$to $\tau$ ".

In a similar way to Lemma 3.17 , we can prove the following lemma:

Lemma 3.25. $\mathfrak{A}_{m}^{p} \models \varphi_{p}$ and $\mathfrak{B}_{m}^{p} \not \models \varphi_{p}$.

Lemma 3.26. For any first-order sentence $\zeta$ over $\tau$, there is a first-order sentence $\xi$ over $\tau^{+}$, with the same quantifier structure, such that the following hold:

(1) $\mathfrak{A}_{m}^{p}=\zeta$ iff $\widetilde{\mathfrak{A}}_{m}^{p} \models \xi$;

(2) $\mathfrak{B}_{m}^{p}=\zeta$ iff $\widetilde{\mathfrak{B}}_{m}^{p}=\xi$.

Proof. Let $\xi$ be obtained from $\zeta$ by

(a) relativising all quantifiers in $\zeta$ by $x \neq r$;

(b) replacing all occurrences of Exy by

$$
R x y \vee B y x \vee(R r x \wedge x=y) \vee(R r x \wedge U(y)) \vee(R r y \wedge U(x)) .
$$

Note that the quantifier structure of $\xi$ is the same as that of $\zeta$.

Because $\mathfrak{A}_{m}^{p}$ is obtained from $\widetilde{\mathfrak{A}}_{m}^{p}$ by:

- deleting the root (corresponding to relativising quantifiers);

- substituting red edges with forward arcs (corresponding to the disjunct $R x y$ in the replacing of Exy); 
- substituting blue edges with backward arcs (corresponding to the disjunct Bxy in the replacing of Exy);

- adding self-loops at the junction points that are connected to the root(corresponding to the disjunct $\operatorname{Rr} x \wedge x=y$ in the replacing of Exy);

- adding bi-directional edges between the black leaves and junction points that are in the same connected component (corresponding to the disjuncts $(\operatorname{Rr} x \wedge U(y))$ and $(\operatorname{Rr} y \wedge U(x))$ in the replacing of Exy),

it gives a reduction from the property defined by $\xi$ to the property defined by $\zeta$. In other words, $\mathfrak{A}_{m}^{p}=\zeta$ iff $\widetilde{\mathfrak{A}}_{m}^{p} \models \xi$. For the same reason, (2) also holds.

Now, we prove the main result in this section, i.e. the theorem 3.1.

Proof. Let $p \in \mathscr{W}\left(S_{1}\right) \backslash \mathscr{W}\left(S_{2}\right)$. Clearly, the property definable by $\varphi_{p}$ is in $\operatorname{FO}\left\{S_{1}\right\}$. We try to prove that this property is not in $\mathrm{FO}\left\{S_{2}\right\}$. Assume on the contrary that there is a formula $\psi$ such that $q s(\psi) \preceq_{e} S_{2}$ and $\psi$ defines the same property as $\varphi_{p}$ does. And let $q r(\psi)$ be $m$. According to Lemma 3.25, $\mathfrak{A}_{m}^{p}=\psi$ and $\mathfrak{B}_{m}^{p} \not \models \psi$. According to Lemma 3.26, there is $\xi$, with the same quantifier structure as $\psi$, such that $\widetilde{\mathfrak{A}}_{m}^{p} \models \xi$ and $\widetilde{\mathfrak{B}}_{m}^{p} \not \xi$. Note that $\mathscr{W}(q s(\xi)) \subseteq \gamma^{-}(f(p))$ since $q s(\xi) \preceq_{e} S_{2}$ and $\mathscr{W}\left(S_{2}\right) \subseteq \gamma^{-}(f(p))$. But this is in contradiction to Lemma 3.22 .

Theorem 3.1 tells us that the distinctive collections of quantifier classes form a strict hierarchy, which we call quantifier structure hierarchy.

\section{Strictness of QUANTIFIER HIERARChy OVER ORDERED Finite STRUCTURES}

Up to now, using logics to characterize complexity classes inside NP requires the structures to be ordered, i.e. there is a linear order over the universe of the structures. Therefore, it is interesting to extend the main result in the last section to ordered structures: the first-order quantifier structure hierarchy is strict over ordered finite structures. However, separating the expressive power of logics over ordered structures is often difficult, because the spoiler may detect the difference between the structures using a given linear order. But we will see in this section that the structures will be constructed in such a way that the power of linear order that the spoiler can use is quite limited: it is equivalent to the power that the spoiler can use in a game over a pair of linear orders, and a well-known result tells us that the duplicator has a winning strategy over a game between two linear orders that are sufficiently long. In this section we sketch the main ideas that conquer the order problem and omit most details that resemble those in the formal proof of Theorem 3.1 .

\subsection{The constructions and separating property.}

Definition 4.1. Recall that $\tau^{+\mathrm{ORD}}$ is $\langle R, B, U, r, \leq\rangle$ where $\leq$ is interpreted as a linear order over the universe. Let $\tau_{o}^{\prime}:=\tau^{+\mathrm{ORD}} \backslash\{U\}$.

Let the structure $\overrightarrow{\mathfrak{A}}_{m}^{p}$ be a $\tau^{+\mathrm{ORD}}$-structure defined as follows:

- $\overrightarrow{\mathfrak{A}}_{m}^{p} \mid \tau^{+}$and $\overrightarrow{\mathfrak{B}}_{m}^{p} \mid \tau^{+}$are trees, whose roots interpret $r$, and whose edges are coloured either red or blue.

- If $p=\exists$, 
(1) $\overrightarrow{\mathfrak{A}}_{m}^{p} \mid \tau^{+}$is a depth 1 tree that has $2^{m+2}+1$ leaves. To construct $\overrightarrow{\mathfrak{A}}_{m}^{p}$ from $\overrightarrow{\mathfrak{A}}_{m}^{p} \mid \tau^{+}$, give these leaves some order such that the $\left(2^{m+1}+1\right)$-th leaf is black. All the other leaves are not black.

(2) $\overrightarrow{\mathfrak{B}}_{m}^{p} \mid \tau^{+}$is a depth 1 tree that has $2^{m+1}$ leaves. None of them is black. Give these leaves an arbitrary order to construct $\overrightarrow{\mathfrak{B}}_{m}^{p}$ from $\overrightarrow{\mathfrak{B}}_{m}^{p} \mid \tau^{+}$.

- If $p=\forall$, Let $\overrightarrow{\mathcal{T}}_{\exists, m}:=\overrightarrow{\mathfrak{A}}_{m}^{\exists}\left|\tau_{o}^{\prime} ; \overrightarrow{\mathcal{T}}_{\exists, m}:=\overrightarrow{\mathfrak{B}}_{m}^{\exists}\right| \tau_{o}^{\prime}$.

(1) $\overrightarrow{\mathfrak{A}}_{m}^{p} \mid \tau^{+}$is a depth 1 tree that has $2^{m+1}$ leaves. All of them are black.

(2) $\overrightarrow{\mathfrak{B}}_{m}^{p} \mid \tau^{+}$is a depth 1 tree that has $\left(2^{m+2}+1\right)$ leaves. All are black except one. Give these leaves some order such that the $\left(2^{m+1}+1\right)$-th leaf is not black.

In the above definition, we let a node be earlier in the linear order $\leq$ than its children. Moreover, we define all the colours of the edges to be red when $|p|=1$.

- When $|p|>1$ :

Let $\overrightarrow{\mathfrak{A}}_{m}^{-q}$ be the same as $\overrightarrow{\mathfrak{A}}_{m}^{q}$ except that the colours of all the edges are exchanged, i.e. red is changed to blue and vice versa. Let $\overrightarrow{\mathfrak{D}}_{q, m}^{A, B}$, a $\left(\tau^{+\mathrm{ORD}} \cup\{\mathfrak{e}\} \backslash\{r\}\right)$-structure, be built from a copy of $\overrightarrow{\mathfrak{A}}_{m}^{q}$ and a copy of $\overrightarrow{\mathfrak{B}}_{m}^{-q}$ where they are joined together at their roots. Their shared root is called junction point, which is used to interpretes the constant $\mathfrak{e}$. Similarly define $\overrightarrow{\mathfrak{D}}_{q, m}^{A, A}$ as the join of $\overrightarrow{\mathfrak{A}}_{m}^{q}$ and $\overrightarrow{\mathfrak{A}}_{m}^{-q}$, define $\overrightarrow{\mathfrak{D}}_{q, m}^{B, A}$ as the join of $\overrightarrow{\mathfrak{B}}_{m}^{q}$ and $\overrightarrow{\mathfrak{A}}_{m}^{-q}$, and define $\overrightarrow{\mathfrak{D}}_{q, m}^{B, B}$ as the join of $\overrightarrow{\mathfrak{B}}_{m}^{q}$ and $\overrightarrow{\mathfrak{B}}_{m}^{-q}$. In $\overrightarrow{\mathfrak{D}}_{q, m}^{A, B}$, we let the elements in $\overrightarrow{\mathfrak{A}}_{m}^{q}$ be later in the linear order than the elements in $\overrightarrow{\mathfrak{B}}_{m}^{-q}$. Similarly, in $\overrightarrow{\mathfrak{D}}_{q, m}^{A, A}$, we let the elements in $\overrightarrow{\mathfrak{A}}_{m}^{q}$ be later in the order than the elements in $\overrightarrow{\mathfrak{A}}_{m}^{-q}$; in $\overrightarrow{\mathfrak{D}}_{q, m}^{B, A}$, the elements in $\overrightarrow{\mathfrak{B}}_{m}^{q}$ are later in the order than the elements in $\overrightarrow{\mathfrak{A}}_{m}^{-q}$; in $\overrightarrow{\mathfrak{D}}_{q, m}^{B, B}$, the elements in $\overrightarrow{\mathfrak{B}}_{m}^{q}$ are later in the order than the elements in $\overrightarrow{\mathfrak{B}}_{m}^{-q}$.

In the following, we assume that any node in $\overrightarrow{\mathfrak{D}}_{q, m}^{X_{1}, Y_{1}}$ is later in the order than all nodes in $\overrightarrow{\mathfrak{D}}_{q, m}^{X_{2}, Y_{2}}$ if the junction point of $\overrightarrow{\mathfrak{D}}_{q, m}^{X_{1}, Y_{1}}$ is later in the order than the junction point of $\overrightarrow{\mathfrak{D}}_{q, m}^{X_{2}, Y_{2}}$.

- If $p=\exists q$,

$\left(1^{\exists}\right) \overrightarrow{\mathfrak{A}}_{m}^{p}$ is a point-expansion of $\overrightarrow{\mathcal{T}}_{\mathfrak{A}, m}$ over $\left\{\mathcal{I}, \overrightarrow{\mathfrak{D}}_{q, m}^{A, A}, \overrightarrow{\mathfrak{D}}_{q, m}^{A, B}, \overrightarrow{\mathfrak{D}}_{q, m}^{B, A}\right\}$. Its root is expanded by $\mathcal{I}$. Recall that $\mathcal{I}$ is a structure over the signature $\left\langle c_{I}\right\rangle$, whose universe has exactly one element, and this element is used to interpret the constant $c_{I}$. When $i \leq 2^{m+1}$, the $i$-th leaf is expanded by a copy of $\overrightarrow{\mathfrak{D}}_{q, m}^{A, B}$ if $i$ is odd, by a copy of $\overrightarrow{\mathfrak{D}}_{q, m}^{B, A}$ if $i$ is even; the $i$-th leaf is expanded by a copy of $\overrightarrow{\mathfrak{D}}_{q, m}^{A, A}$ if $i=2^{m+1}+1$; when $i>2^{m+1}+1$, the $i$-th leaf is expanded by a copy of $\overrightarrow{\mathfrak{D}}_{q, m}^{A, B}$ if $i-\left(2^{m+1}+1\right)$ is odd, by a copy of $\overrightarrow{\mathfrak{D}}_{q, m}^{B, A}$ if $i-\left(2^{m+1}+1\right)$ is even.

$\left(2^{\exists}\right) \overrightarrow{\mathfrak{B}}_{m}^{p}$ is a point-expansion of $\overrightarrow{\mathcal{T}}_{\exists, m}^{\mathfrak{B}}$ over $\left\{\mathcal{I}, \overrightarrow{\mathfrak{D}}_{q, m}^{A, B}, \overrightarrow{\mathfrak{D}}_{q, m}^{B, A}\right\}$. Its root is expanded by $\mathcal{I}$. The $i$-th leaf is expanded by a copy of $\overrightarrow{\mathfrak{D}}_{q, m}^{A, B}$ if $i$ is odd, by a copy of $\overrightarrow{\mathfrak{D}}_{q, m}^{B, A}$ if $i$ is even.

- If $p=\forall q$,

$11^{\forall}$ ) The lem of $\overrightarrow{\mathfrak{A}}_{m}^{p}$ is the same as the above lem $\left(2^{\exists}\right)$ of $\overrightarrow{\mathfrak{B}}_{m}^{\exists q}$.

$21^{\forall}$ ) The lem of $\overrightarrow{\mathfrak{B}}_{m}^{p}$ is the same as the lem $\left(1^{\exists}\right)$ of $\overrightarrow{\mathfrak{A}}_{m}^{\exists q}$ except that $\overrightarrow{\mathfrak{D}}_{q, m}^{A, A}$ is replaced by a copy of $\overrightarrow{\mathfrak{D}}_{q, m}^{B, B}$. 
Note that every structure under consideration is just an ordered coloured tree and there is a path from its root to any node. We can read off the string of colours of edges along this path without a skip. Define $l a b(x)$ to be a string in $\{\text { red, blue }\}^{*}$ associated with the path. $l a b(r):=\epsilon$, where $\epsilon$ is the empty string. We let "red" be later in the order than "blue" in the lexicographic order. Let $x, y$ be two nodes. We use $x^{\mathfrak{f}}$ ( $y^{\mathfrak{f}}$ resp.) to denote the father of $x$ (y resp.) in this section.

In the above, we have defined the structures that will be used in the games. And the sentence that we use to define the separating property is the same as Definition 3.15. That is, we actually use the same separating property to achieve the goal.

In the last section, we use point-expansions to realize strategy compositions. In some special cases, such compositions can be simplified: some of the substructures collapse to "points", i.e. the details of the substructures are omitted, and we use "colours" to distinguish different substructures, which are now regarded temporarily as elements. We call such a method a kind of "structural abstraction", which is used to omit unrelated details of structures and simplify game arguments, and the games played on the simplified structures are images of the original games.

4.2. The duplicator's winning strategy. Note that, even in the unordered case, when the spoiler picks $x$, the duplicator's strategy in the games is always picking $y$ s.t. $l a b(x)=l a b(y)$ and picking a child of an element which is picked in some previous round, say the $i$-th round, when the spoiler picks a child of the other element which is picked in the $i$-th round. This lays the crucial basis for the previous inductive proof of Theorem 3.23 to extend to classes of linearly ordered finite structures, because now we can use something similar to the following well-known result [4]:

Let $k \geq 1$, and let $L_{1}, L_{2}$ be linear orders of length no less than $2^{k}$, then

$$
L_{1} \equiv_{k} L_{2} \text {. }
$$

Here, $L_{1} \equiv_{k} L_{2}$ means that, for any $\varphi \in \mathrm{FO}$ with $\operatorname{qr}(\varphi) \leq k, L_{1} \models \varphi$ iff $L_{2}=\varphi$.

Let $\widetilde{\varphi}_{p}$ be given by the definition 3.15. The proof of lemma 3.17 also shows that $\overrightarrow{\mathfrak{A}}_{m}^{p}=\widetilde{\varphi}_{p}$ and $\overrightarrow{\mathfrak{B}}_{m}^{p} \not \widetilde{\varphi}_{p}$, since " $\leq$ " does not appear in $\widetilde{\varphi}_{p}$.

To prove a version of Theorem 3.23 over ordered structures, the main idea is almost the same. Here, we just need to take care of the linear order. As we have explained before, the players will always pick a pair of elements that have the same label, and if the spoiler picks more than one child of a node in one structure, so does the duplicator in the other structure. Hence, we can use structural abstraction to conceal the details of the substructures like $\overrightarrow{\mathfrak{D}}_{q, m}^{A, B}$, and regard the problem to be a game over two linear orders with three "colours", which represent three "colours" $\overrightarrow{\mathfrak{D}}_{q, m}^{A, B}, \overrightarrow{\mathfrak{D}}_{q, m}^{B, A}$, and $\overrightarrow{\mathfrak{D}}_{q, m}^{A, A}$ respectively. See Figure 5 for example. Here, $\overrightarrow{\mathfrak{D}}_{q, m}^{A, B}$ is identified with light red; $\overrightarrow{\mathfrak{D}}_{q, m}^{B, A}$ is identified with light blue; $\overrightarrow{\mathfrak{D}}_{q, m}^{A, A}$ is identified with green.

As explained before, we may safely assume that $p[1]=\exists$.

Assume that $p=\exists q$. We can pair the children of $r^{\overrightarrow{\mathfrak{A}}_{m}^{p}}$ as:

$$
\left(\overrightarrow{\mathfrak{D}}_{q, m}^{A, B}, \overrightarrow{\mathfrak{D}}_{q, m}^{B, A}\right), \cdots,\left(\overrightarrow{\mathfrak{D}}_{q, m}^{A, A}, \times\right), \cdots,\left(\overrightarrow{\mathfrak{D}}_{q, m}^{A, B}, \overrightarrow{\mathfrak{D}}_{q, m}^{B, A}\right) .
$$

We can regard two $\left(\tau^{+ \text {ORD }} \cup\{\mathfrak{e}\} \backslash\{r\}\right)$-structures in the brackets as a whole. More precisely, an $s$-2-tuple is a pair of structures from $\left\{\overrightarrow{\mathfrak{D}}_{s, m}^{A, B}, \overrightarrow{\mathfrak{D}}_{s, m}^{B, A}, \overrightarrow{\mathfrak{D}}_{s, m}^{A, A}, \overrightarrow{\mathfrak{D}}_{s, m}^{B, B}\right\}(s \preceq p)$, which is regarded as a single "super-element". From now on, we omit " $s$ " in " $s$-2-tuple" when it 

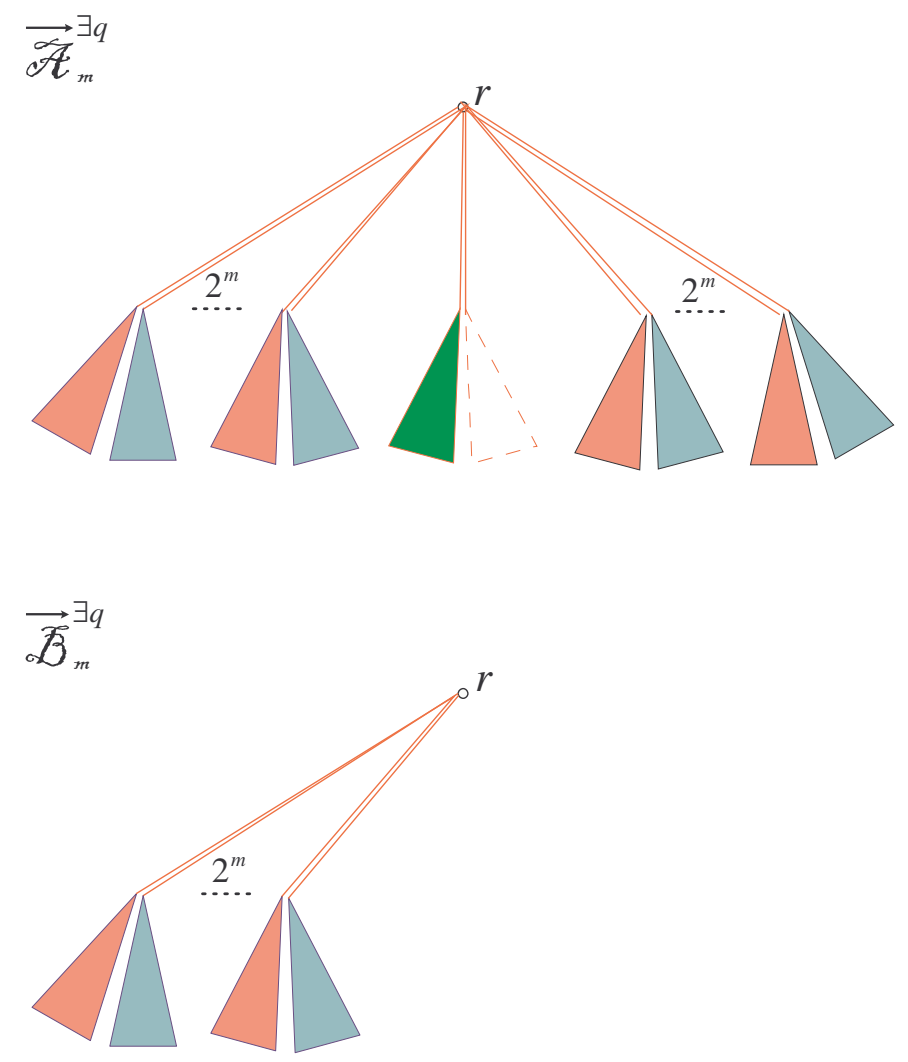

Figure 5: The structures where subgraphs are identified with "colours".

will not cause confusion from the context. In 4.1), " $\times$ " in the 2 -tuple $\left(\mathfrak{D}_{q, m}^{A, A}, \times\right)$ represents an empty, or imaginary, structure, which is just used to make up a 2-tuple. See Figure 6. But, since we are regarding a 2-tuple as one single object now, we use one dashed line to represent two arrows. Note that, by definition, the elements of $\overrightarrow{\mathfrak{D}}_{s, m}^{A, B}$ are earlier than those of $\overrightarrow{\mathfrak{D}}_{s, m}^{B, A}$, in a 2-tuple $\left(\overrightarrow{\mathfrak{D}}_{s, m}^{A, B}, \overrightarrow{\mathfrak{D}}_{s, m}^{B, A}\right)$. These 2-tuples have a natural linear order $\leq_{2}$ inherited from $\leq^{\overrightarrow{\mathfrak{A}}_{m}^{p}}$ : for any two 2-tuples $\left(\mathcal{X}_{1}, \mathcal{Y}_{1}\right)$ and $\left(\mathcal{X}_{2}, \mathcal{Y}_{2}\right),\left(\mathcal{X}_{1}, \mathcal{Y}_{1}\right) \leq_{2}\left(\mathcal{X}_{2}, \mathcal{Y}_{2}\right)$ iff $r^{\mathcal{Y}_{1}} \leq^{\overrightarrow{\mathfrak{A}}_{m}^{p}} r^{\mathcal{X}_{2}}$ where $r^{\mathcal{Y}_{1}}$ and $r^{\mathcal{X}_{2}}$ are the junction points of $\mathcal{Y}_{1}$ and $\mathcal{X}_{2}$ respectively. Similarly, we can pair the children of $r^{\overrightarrow{\mathfrak{B}}_{m}^{p}}$. Note that there are $\left(2^{m+1}+1\right) 2$-tuples in $\overrightarrow{\mathfrak{A}}_{m}^{p}$, which form a linear order $L_{A}$, and $2^{m} 2$-tuples in $\overrightarrow{\mathfrak{B}}_{m}^{p}$, which form another linear order $L_{B}$. See Figure 7; a yellow node represents a 2-tuple, which corresponds to a pair of light red and light blue structures in Figure 5; the green node represents the 2-tuple that is green in Figure 5.

Let $S$ be a $\Gamma$-labelled forest and $r k(S)=m$. In the game $G_{S}\left(\overrightarrow{\mathfrak{A}}_{m}^{p}, \overrightarrow{\mathfrak{B}}_{m}^{p}\right)$, when the spoiler picks an element $x$, which is not the root of the structure, in some round, $x$ determines a $q$-2-tuple, where $p=\exists q$, s.t. $x$ is in the universe of this $q$-2-tuple. And we may also say that this 2 -tuple is picked in this round. Hence, $G_{S}\left(\overrightarrow{\mathfrak{A}}_{m}^{p}, \overrightarrow{\mathfrak{B}}_{m}^{p}\right)$ induces a new game in which the 

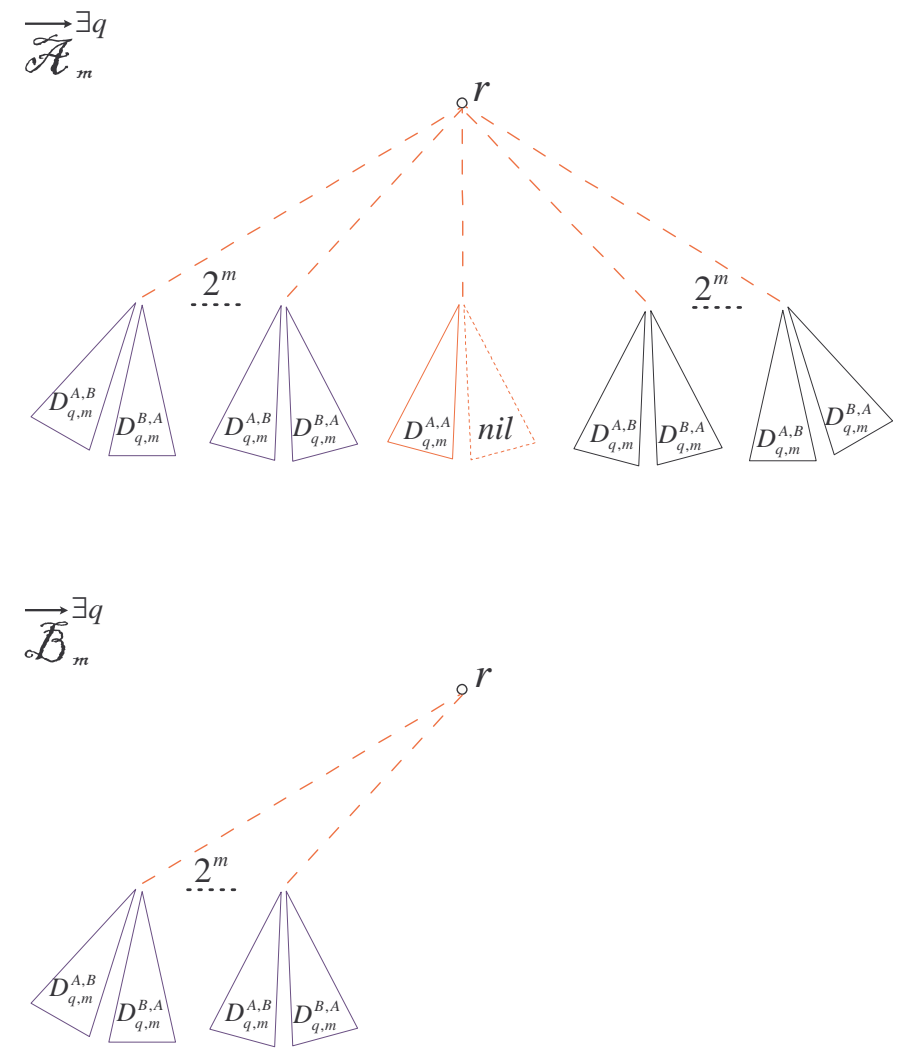

Figure 6: The structures and 2-tuples.

players pick 2-tuples instead of elements in the universe. Moreover, these 2-tuples form a pair of linear orders $L_{A}$ and $L_{B}$, as shown in Figure 7. Call this new game "coloured linear order game" $G_{S}\left(L_{A}, L_{B}\right)$.

From now on, we use a natural number to denote a 2-tuple, in order to omit the details of 2-tuples that are not related to our concern but at the same time retain the order relation between 2-tuples. Therefore, we can subtract one 2-tuple from another 2-tuple in this context. Note that in this viewpoint linear orders can also be regarded as intervals. Moreover, every set of elements that have the same labels form an interval.

The duplicator's strategy in $G_{S}\left(L_{A}, L_{B}\right)$ is as follows. Assume that $\mathcal{B}_{i}, \mathcal{B}_{j}$ are already picked. Let $\mathcal{A}_{i}\left(\mathcal{A}_{j}\right.$ resp.) be the element picked in $L_{A}$ in the same round as $\mathcal{B}_{i}$ ( $\mathcal{B}_{j}$ resp.) was picked. Recall that these 2-tuples can be compared by the induced order.

- If in the current round the spoiler picks a 2-tuple $\mathcal{B}$ in the interval $\left[\mathcal{B}_{i}, \mathcal{B}_{j}\right]$ and $\mathcal{B}-\mathcal{B}_{i} \leq$ $\mathcal{B}_{j}-\mathcal{B}$ then the duplicator picks $\mathcal{A}$ s.t. $\mathcal{A}-\mathcal{A}_{i}=\mathcal{B}-\mathcal{B}_{i}$; otherwise she picks $\mathcal{A}$ s.t. $\mathcal{A}_{j}-\mathcal{A}=\mathcal{B}_{j}-\mathcal{B}$.

- If in the current round the spoiler picks a 2-tuple $\mathcal{A}$ in the interval $\left[\mathcal{A}_{i}, \mathcal{A}_{j}\right]$, the duplicator's strategy is as follows: 

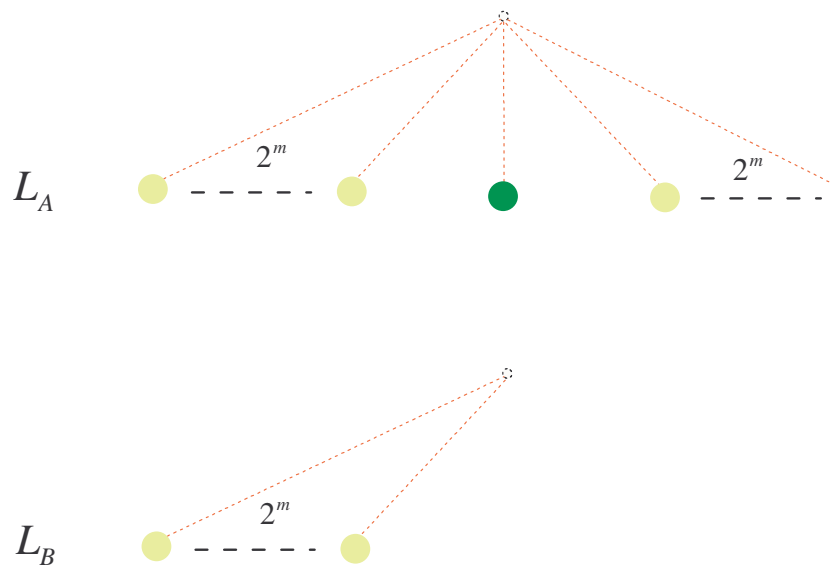

Figure 7: $L_{A}$ and $L_{B}$.

(1) if $\mathcal{A}-\mathcal{A}_{i} \leq \frac{1}{2}\left(\mathcal{B}_{j}-\mathcal{B}_{i}\right)$ then the duplicator picks $\mathcal{B}$ in $\left[\mathcal{B}_{i}, \mathcal{B}_{j}\right]$ s.t. $\mathcal{B}-\mathcal{B}_{i}=\mathcal{A}-\mathcal{A}_{i}$; otherwise

(2) if $\mathcal{A}_{j}-\mathcal{A} \leq \frac{1}{2}\left(\mathcal{B}_{j}-\mathcal{B}_{i}\right)$ then the duplicator picks $\mathcal{B}$ in $\left[\mathcal{B}_{i}, \mathcal{B}_{j}\right]$ s.t. $\mathcal{B}_{j}-\mathcal{B}=\mathcal{A}_{j}-\mathcal{A}$; otherwise

(3) the duplicator picks the middle element in $\left[\mathcal{B}_{i}, \mathcal{B}_{j}\right]$ : if the special 2-tuple $\left(\overrightarrow{\mathfrak{D}}_{q, m}^{A, A}, \times\right)$ (the green node in Figure 7 is in the region $\left[\mathcal{A}, \mathcal{A}_{j}\right]$ then $\mathcal{B}-\mathcal{B}_{i}=\left\lfloor\frac{1}{2}\left(\mathcal{B}_{j}-\mathcal{B}_{i}\right)\right\rfloor$, otherwise $\mathcal{B}_{j}-\mathcal{B}=\left\lfloor\frac{1}{2}\left(\mathcal{B}_{j}-\mathcal{B}_{i}\right)\right\rfloor$.

Assume that in the first $k$ rounds the spoiler is restricted to pick in $\overrightarrow{\mathfrak{B}}_{m}^{p}$.

Because a 2-tuple may contain smaller 2-tuples when looking inside it, this strategy can be applied recursively until the duplicator finds an element to pick in the game $G_{S}\left(\overrightarrow{\mathfrak{A}}_{m}^{p}, \overrightarrow{\mathfrak{B}}_{m}^{p}\right)$ : she always picks an element that has the same label as the element picked by the spoiler in the same round. Using this strategy together with the strategy that is used in the game between two unordered structures, the duplicator can ensure that $\left(\overrightarrow{\mathfrak{D}}_{q, m}^{A, A}, \times\right)$ will never be picked in the first $k$ rounds, in which the spoiler picks 2-tuples in $L_{B}$, and order itself will not cause a problem throughout the game $G_{S}\left(\overrightarrow{\mathfrak{A}}_{m}^{p}, \overrightarrow{\mathfrak{B}}_{m}^{p}\right)$. More precisely, the following two lemmas are true: (Recall that $S$ is a $\Gamma$-labelled forest, and $m=r k(S)$ )

Lemma 4.2. Assume that $k \in[0, m]$, and in the first $k$ rounds of the game $G_{S}\left(L_{A}, L_{B}\right)$, the spoiler picks 2-tuples in $L_{B}$.

(i) At the end of the $i$-th round of $G_{S}\left(L_{A}, L_{B}\right)$, for each $i \leq k$, there is only one interval in $L_{A}$ that is not isomorphic to the corresponding interval in $L_{B}$, both of which are no shorter than $2^{m-i}$, and $\left(\overrightarrow{\mathfrak{D}}_{q, m}^{A, A}, \times\right)$ always lies in this interval of $L_{A}$.

(ii) In the game $G_{S}\left(L_{A}, L_{B}\right)$, for any $i, j \leq k$,

$$
\mathcal{A}_{i} \leq_{2} \mathcal{A}_{j} \text { iff } \mathcal{B}_{i} \leq_{2} \mathcal{B}_{j}
$$


Proof. Before the game $G_{S}\left(L_{A}, L_{B}\right)$, there is only one interval in $L_{A}$ and $L_{B}$. Namely $L_{A}$ and $L_{B}$ themselves. Let $\mathcal{A}_{f}, \mathcal{A}_{l}$ be the fist and the last 2-tuples of $L_{A}$ respectively. And $\mathcal{B}_{f}$ and $\mathcal{B}_{l}$ be the first and the last 2-tuples of $L_{B}$ respectively.

(1) In the first round, there are two possibilities:

(a) The players pick the first 2-tuple or the last 2-tuple in the respective intervals and the intervals remain unchanged;

(b) The picked 2-tuples split $L_{A}$ and $L_{B}$ into two intervals. In this case, according to the duplicator's strategy, one interval in $L_{A}$ and $L_{B}$ has the same length, which is no larger than $2^{m-1}$, and the other interval in $L_{A}$ includes $\left(\overrightarrow{\mathfrak{D}}_{q, m}^{A, A}, \times\right)$ because the length between the first 2-tuple (the last 2-tuple resp.) and $\left(\overrightarrow{\mathfrak{D}}_{q, m}^{A, A}, \times\right)$ is the length of $L_{B}$ plus one which means that this length is larger than any interval in $L_{B}$. It also means that the interval which includes $\left(\overrightarrow{\mathfrak{D}}_{q, m}^{A, A}, \times\right)$ is longer than the other one in $L_{A}$. Hence, it is larger than $2^{m-1}$, like the corresponding interval in $L_{B}$.

Therefore, (i) holds when the game is at the end of the first round. Assume that (i) holds when the game is at the end of the $s$-th round, where $1 \leq s \leq k$.

When the game is in the $(s+1)$-th round, there are only two cases:

(a) The spoiler picks a 2-tuple that is in the interval, say $I_{b}$ in $L_{B}$. Let the corresponding interval in $L_{A}$ be $I_{a}$, which includes the 2-tuple $\left(\overrightarrow{\mathfrak{D}}_{q, m}^{A, A}, \times\right)$. By assumption, both of them are longer than $2^{m-s}$. In this round, the unique pair of intervals $I_{a}$ and $I_{b}$ are split into two pairs: one is isomorphic; the other one is not and includes $\left(\overrightarrow{\mathfrak{D}}_{q, m}^{A, A}, \times\right)$ because $\left(\overrightarrow{\mathfrak{D}}_{q, m}^{A, A}, \times\right)$ splits $I_{a}$ into two pieces and both of them are longer than $I_{b}$. Note that the pair of isomorphic intervals are no longer than $2^{m-s-1}$. Hence, the other pair of intervals are no shorter than $2^{m-s-1}$, by the duplicator's strategy.

(b) The spoiler picks a 2-tuple in other intervals. Note that all the other pairs of intervals have the same length. According to the duplicator's strategy, splitting such a pair of intervals only produces pairs of intervals of the same length. And the pair of intervals which are not isomorphic is unchanged. By the inductive assumption, (i) still holds.

(2) We prove an equivalent conclusion, i.e. (ii) holds if we add two rounds before the game in which $\mathcal{A}_{f}, \mathcal{A}_{l}, \mathcal{B}_{f}$ and $\mathcal{B}_{l}$ are picked. Clearly, $\mathcal{A}_{f} \leq_{2} \mathcal{A}_{l}$ iff $\mathcal{B}_{f} \leq_{2} \mathcal{B}_{l}$. In other words, it holds when these two elements are picked.

Suppose (ii) holds when the game is at the end of the $s$-th round, where $s \geq 0$.

Now assume that the game is in the $(s+1)$-th round of the game. If the spoiler picks a 2-tuple that was picked before, then it still holds. If the spoiler picks a 2-tuple $\mathcal{B}$ that splits some interval in $L_{B}$, say $\left[\mathcal{B}_{h}, \mathcal{B}_{t}\right]$, the duplicator also picks a 2 -tuple $\mathcal{A}$ that splits the corresponding interval $\left[\mathcal{A}_{h}, \mathcal{A}_{t}\right]$ by her strategy. If $\left[\mathcal{B}_{h}, \mathcal{B}_{t}\right]$ and $\left[\mathcal{A}_{h}, \mathcal{A}_{t}\right]$ are isomorphic, then obviously (ii) holds. If it is not the case, due to (i), we know that $\left[\mathcal{A}_{h}, \mathcal{A}_{t}\right]$ is sufficiently long such that it allows such splitting. Then for any 2-tuple $\mathcal{B}_{x}$ that is picked before, $\mathcal{B}_{x} \leq_{2} \mathcal{B}_{h}$ if $\mathcal{B}_{x} \leq_{2} \mathcal{B}$, which, by assumption, implies that $\mathcal{A}_{x} \leq_{2} \mathcal{A}_{h}$. Hence, $\mathcal{A}_{x} \leq_{2} \mathcal{A}$. Likewise, $\mathcal{A}_{x} \geq_{2} \mathcal{A}$ if $\mathcal{B}_{x} \geq_{2} \mathcal{B}$. Therefore, for any $i, j$, $\mathcal{A}_{i} \leq_{2} \mathcal{A}_{j}$ if $\mathcal{B}_{i} \leq_{2} \mathcal{B}_{j}$. The "only if" part can be proven similarly.

Part (i) of Lemma 4.2 tells us that the spoiler cannot use the linear order to force the duplicator to violate her winning strategy in $G_{S}\left(\mathfrak{A}_{m}^{p}, \mathfrak{B}_{m}^{p}\right)$, no matter how he picks in $L_{B}$. That is, if the spoiler picks a yellow node in $L_{B}$ (see Figure 7 ), the duplicator can also pick a yellow node. A similar thing can be proved when $p[1]=\forall$. Because the collection of 2-tuples 
whose roots are children (with the same label) of an element (node) in $\overrightarrow{\mathfrak{A}}_{m}^{p}$ form an interval, we can generalize (ii) of Lemma 4.2 such that it applies to any pair of intervals that are split in the same round. Recall that a 2-tuple is composed of two trees. A 2-tuple is at depth $i$ if the roots of trees of this 2-tuple are at depth $i$. Hence, we can call these intervals intervals at depth $i$ if the elements (2-tuples) of these intervals are at depth $i$ of the structures (trees).

Lemma 4.3. Let $S$ be a $\Gamma$-labelled forest and $m=r k(S)$. Let $p$ be a prefix. In the game $G_{S}\left(\overrightarrow{\mathfrak{A}}_{m}^{p}, \overrightarrow{\mathfrak{B}}_{m}^{p}\right)$ where $p \notin \mathscr{W}(S)$, the duplicator can play in such a way that:

(i) she follows her winning strategy in $G_{S}\left(\mathfrak{A}_{m}^{p}, \mathfrak{B}_{m}^{p}\right)$ that has been described in Lemma 3.2.:

(ii) for any $a_{i}, a_{j} \in\left|\overrightarrow{\mathfrak{A}}_{m}^{p}\right|, b_{i}, b_{j} \in\left|\overrightarrow{\mathfrak{B}}_{m}^{p}\right|$, which are picked by the players in $i$-th and $j$-th rounds $(i, j \leq m)$,

$$
a_{i} \leq^{\overrightarrow{\mathfrak{A}}_{m}^{p}} a_{j} \text { iff } b_{i} \leq^{\overrightarrow{\mathfrak{B}}_{m}^{p}} b_{j}
$$

Proof. We assume that, before the first round of the game, both the first child and last child of any inner node are already picked. It means that we are trying to prove an equivalent result.

In each round, when the spoiler picks an element $a$ in a structure, say $\overrightarrow{\mathfrak{A}}_{m}^{p}$, there is a path $P$ from the root of the tree $\overrightarrow{\mathfrak{A}}_{m}^{p}$ to the node $a$. For any node $v$ in the path $P$ there is a 2-tuple $(\mathcal{X}, \mathcal{Y})$ such that $(\mathcal{X}, \mathcal{Y})$ is the 2-tuple that include $a$ and $v$ is the root of either $\mathcal{X}$ or $\mathcal{Y}$. Let $a$ be at the depth $r$ of the tree $\overrightarrow{\mathfrak{A}}_{m}^{p}$ and let the path $P$ be $\left(a_{0}, \cdots, a_{r}\right)$ where $a_{0}$ is the root of $\overrightarrow{\mathfrak{A}}_{m}^{p}$ and $a_{r}=a$. Assume that the intervals of 2 -tuples that are split by the path is $\left(L_{1}^{A}, \cdots, L_{r}^{A}\right)$. That is, $L_{i}^{A}$ is an interval at the depth $i$ of the tree $\overrightarrow{\mathfrak{A}}_{m}^{p}$, which includes $a_{i}$. Let $\left(L_{1}^{B}, \cdots, L_{r}^{B}\right)$ be the collection of intervals of 2-tuples where $L_{i}^{B}$ is at depth $i$ and is formed in the same rounds as $L_{i}^{A}$. The duplicator first picks all the nodes in the path $\left(a_{0}, \cdots, a_{r}\right)$ (Looking at it in another way, the spoiler implicitly picks all the nodes in the path). Then she uses her strategy in coloured linear order games recursively as follows: she first plays the one round coloured linear order game at the depth 1, i.e. over the pair of intervals $\left(L_{1}^{A}, L_{1}^{B}\right)$, picking a 2-tuple at the depth 1 of $\overrightarrow{\mathfrak{B}}_{m}^{p}$, which splits the interval $L_{1}^{B}$ as a consequence; then she picks the root of a tree in the 2-tuple (recall that a 2-tuple is composed of two trees) which respects her wining strategy in $G_{S}\left(\mathfrak{A}_{m}^{p}, \mathfrak{B}_{m}^{p}\right)$ (recall Definition 3.13 for the definition of $\mathfrak{A}_{m}^{p}$ and $\mathfrak{B}_{m}^{p}$ ): whenever possible she tries to choose the tree, whose root is $b_{1}$, that is isomorphic to the one implicitly picked by the spoiler in $L_{1}^{A}$ whose root is $a_{1}$, and pick $b_{1}$. Then she goes on to play the game over the pair of intervals $\left(L_{2}^{A}, L_{2}^{B}\right)$. For $1 \leq i \leq r-1$, once she picked a 2 -tuple at depth $i$, she will pick the root, say $c$, of a tree from the 2-tuple, afterwards she picks a 2 -tuple at depth $i+1$, whose trees are the children of $c$. At last, she picks a 2-tuple in the interval $L_{r}^{B}$ and picks the root of a tree from this 2-tuple that respects her winning strategy in $G_{S}\left(\mathfrak{A}_{m}^{p}, \mathfrak{B}_{m}^{p}\right)$.

(i) will be violated only when the lengths of the pair of non-isomorphic intervals are not long enough such that the spoiler can force the duplicator to pick a different type of 2-tuple when he picks repeatedly in the shorter interval. Note that any pair of isomorphic intervals have the same type of 2 -tuples. Hence, to prove (i), we need only show that (i') at the end of the $i$-th round, if a pair of intervals is not isomorphic, then the length of them are no less than $2^{m-i}$. However, (i') is obvious because the strategy of the duplicator ensures that after the $i$-th round $(i \geq 1)$, the lengths of any pair of non-isomorphic intervals reduce at most $2^{m-i}$, while they are at least $2^{m-i+1}$ before the $i$-th round, which can be proved inductively 
as in Lemma 4.2. In other words, this strategy is able to incorporate her winning strategy in $G_{S}\left(\mathfrak{A}_{m}^{p}, \mathfrak{B}_{m}^{p}\right)$ - it can guide her to win the game if order is not taken into account. It remains to show that this is a strategy for the duplicator to avoid the order problem, i.e. to show (ii).

For the sake of convenience, we call the stage before the players play the game as the 0 -th round. In the first round, the order will not be a problem since other than those nodes at the ends of intervals, there is no other node that can violate (ii).

Assume that (ii) holds when it is at the end of the $s$-th round.

Now suppose that the game is in the $(s+1)$-th round. Let $a_{i}$ and $a_{j}$ be two elements picked in the $i$-th and $j$-th rounds $(i, j \leq s)$ in $\overrightarrow{\mathfrak{A}}_{m}^{p}$. We may further assume that either $i$ or $j$ equals $s+1$. When $r, a_{i}, a_{j}$ are in a path, then $a_{i}<a_{j}$ implies $b_{i}<b_{j}$ because the duplicator's strategy ensures that $r, b_{i}, b_{j}$ are also in a path.

Now assume that $r, a_{i}, a_{j}$ are not in a path.

If $a_{i}$ and $a_{j}$ have the same father and the same label, which means they are in the same interval, then we can apply the same argument of Lemma 4.2, simply by regarding an element as a 2-tuple. If $a_{i}$ and $a_{j}$ have the same father but have different labels, then by definition their order is determined by their labels. So are $b_{i}$ and $b_{j}$. Note that the label of $a_{i}$ and $b_{i}\left(a_{j}\right.$ and $b_{j}$ resp.) are the same, by the duplicator's strategy. Therefore, (ii) holds.

Assume that $a_{i}$ and $a_{j}$ have different fathers. Note that $a_{i}, a_{j}$ always share at least one ancestor, i.e. the root $r$. Let $c$ be such an shared ancestor, and for all other shared ancestors, $c$ is later in the order. Let $a_{i}^{\prime}$ be the ancestor of $a_{i}$ (or $a_{i}$ itself) and the child of $c$. Let $a_{j}^{\prime}$ be the ancestor of $a_{j}$ (or $a_{j}$ itself) and the child of $c$. Let $b_{i}^{\prime}, b_{j}^{\prime}$ be defined in a similar way in $\overrightarrow{\mathfrak{B}}_{m}^{p}$. Then by definition the order between $a_{i}$ and $a_{j}$ is determined by the order between $a_{i}^{\prime}$ and $a_{j}^{\prime}$. And the duplicator can ensure that $a_{i}^{\prime}<a_{j}^{\prime}$ iff $b_{i}^{\prime}<b_{j}^{\prime}$, according to the same argument as Lemma 4.2. Therefore, (ii) holds.

Lemma 4.3 tell us that linear order does not cause a problem to the duplicator, and together with the arguments in Theorem 3.1 the following holds.

Theorem 4.4. Let $S_{1}$ and $S_{2}$ be two finite $\Gamma$-labelled forests. Over the class of all finite

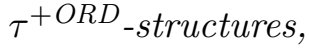

$$
\text { if } \mathscr{W}\left(S_{1}\right) \not \mathscr{W}\left(S_{2}\right) \text {, then } \mathrm{FO}\left\{S_{1}\right\} \nsubseteq \mathrm{FO}\left\{S_{2}\right\} \text {. }
$$

Using similar arguments as in the last section, in particular the same reduction as in Lemma 3.26, we can prove the following Theorem.

Theorem 4.5. Let $S_{1}$ and $S_{2}$ be two finite $\Gamma$-labelled forests. Over the class of all ordered finite digraphs,

$$
\text { if } \mathscr{W}\left(S_{1}\right) \nsubseteq \mathscr{W}\left(S_{2}\right) \text {, then } \mathrm{FO}\left\{S_{1}\right\} \nsubseteq \mathrm{FO}\left\{S_{2}\right\} \text {. }
$$

Here we call $\tau \cup\{\leq\}$-structures (linearly) ordered digraphs.

The following corollary is a special case of Theorem 4.5, where $S_{1}$ and $S_{2}$ are degenerate trees (or directed paths).

Corollary 4.6. Let $p, q \in \Gamma^{*}$. Over the class of all ordered finite digraphs,

$$
\text { if } p \npreceq q \text {, then } \mathrm{FO}\{p\} \nsubseteq \mathrm{FO}\{q\} \text {. }
$$

Note that it is different from Grädel and McColm's conjecture [2]. 
A natural question is whether something similar to Theorem 3.1 holds, but over finite digraphs with built-in BIT. Here, BIT is the binary relation for the bit operator: $\operatorname{BIT}(x, y)=$ 1 if the $y$-th bit of the binary representation of $x$ is 1 . The operator BIT seems very powerful. It is known that first-order logic equipped with BIT can define arbitrary algorithmic operators, including $\leq, \times,+$, Exp, and Squares (Schweikardt, [7]). Supprisingly, Schweikardt and Schwentick [8] showed that BIT is similar to linear orders in terms of expressive power in first-order logic. Based on their constructions, it is not difficult to show that the quantifier structure hierarchy is strict in FO, even in the presence of BIT.

\section{A REFined QUANTIFIER STRUCTURE HIERARChY}

5.1. The structures and separating property. It is possible that two $\Gamma$-labeled forests cannot embed to each other but the set of words that can be read off them are the same. It is natural to conjecture that they represent different logical resources. However, the hierarchy we defined in the last section cannot tell us about it. In the following, we are going to show a refined strict hierarchy, which confirms this intuition:

Theorem 5.1. Let $S_{1}$ and $S_{2}$ be two $\Gamma$-labelled forests. Over the class of all digraphs,

$$
\text { if } S_{1} \npreceq e S_{2} \text {, then } \mathrm{FO}\left\{S_{1}\right\} \nsubseteq \mathrm{FO}\left\{S_{2}\right\} \text {. }
$$

As in the last section, we let $\tau^{+}:=\langle R, B, r, U\rangle$, and let $\tau_{0}^{+}:=\tau^{+} \backslash\{U\}$.

Definition 5.2. A $\Gamma$-labelled tree $\mathcal{T}$ is an irreducible tree if for any inner node $a$ the following holds:

Let $b_{1}, \cdots, b_{k}$ be the children of $a$ and $\mathcal{T}_{1}, \cdots, \mathcal{T}_{k}$ be the maximal subtrees of $\mathcal{T}$ that are rooted at $b_{1}, \cdots, b_{k}$ respectively, then $\mathcal{T}_{i}$ cannot embed in $\mathcal{T}_{j}$ for any $i, j \in[1, k]$ where $i \neq j$.

Definition 5.3. Let $\mathcal{T}$ be a $\Gamma$-labelled irreducible $k$-ary tree.

- $\widetilde{\mathfrak{A}}_{m}^{T}$ and $\widetilde{\mathfrak{B}}_{m}^{T}$ are coloured trees ${ }^{2}$ The constant $r$ is interpreted as the root of the respective trees. As in Definition 3.13, we say an element $a$ is black if $a \in U$.

- (1) $\widetilde{\mathfrak{A}}_{m, l}^{\exists}$ is a depth 1 tree that has $l m+1$ leaves. One of the leaves is black and all the other leaves are not black.

(2) $\widetilde{\mathfrak{B}}_{m, l}^{\exists}$ is a depth 1 tree that has $l m$ leaves. None of them is black.

All edges in $\widetilde{\mathfrak{A}}_{m, l}^{\exists}$ and $\widetilde{\mathfrak{B}}_{m, l}^{\exists}$ are red. Recall that we say an edge $(a, b)$ is red if, and only if, $(a, b) \in R$.

$\mathcal{T}_{m, l}^{\mathfrak{A}, \exists}:=\widetilde{\mathfrak{A}}_{m, l}^{\exists}\left|\tau_{0}^{+} ; \mathcal{T}_{m, l}^{\mathfrak{B}, \exists}:=\widetilde{\mathfrak{B}}_{m, l}^{\exists}\right| \tau_{0}^{+}$.

- (1) $\widetilde{\mathfrak{A}}_{m, l}^{\forall}$ is a depth 1 tree that has $l m$ leaves. All of the leaves are black.

(2) $\widetilde{\mathfrak{B}}_{m, l}^{\forall}$ is a depth 1 tree that has $l m+1$ leaves. One of them is not black and all the other leaves are black.

All edges in $\widetilde{\mathfrak{A}}_{m, l}^{\forall}$ and $\widetilde{\mathfrak{B}}_{m, l}^{\forall}$ are red.

$\mathcal{T}_{m, l}^{\mathfrak{A}, \forall}:=\widetilde{\mathfrak{A}}_{m, l}^{\forall}\left|\tau_{0}^{+} ; \mathcal{T}_{m, l}^{\mathfrak{B}, \forall}:=\widetilde{\mathfrak{B}}_{m, l}^{\forall}\right| \tau_{0}^{+}$.

- If $\mathcal{T}$ contains a single $\mathscr{E}$ node,

$$
\widetilde{\mathfrak{A}}_{m}^{T} \text { is } \widetilde{\mathfrak{A}}_{m, 1}^{\exists} ; \widetilde{\mathfrak{B}}_{m}^{T} \text { is } \widetilde{\mathfrak{B}}_{m, 1}^{\exists} \text {. }
$$

\footnotetext{
${ }^{2}$ Here, the " $T$ " in $\widetilde{\mathfrak{A}}_{m}^{T}$ and $\widetilde{\mathfrak{B}}_{m}^{T}$ refers to the tree $\mathcal{T}$. Also cf. Figure 8
} 
- If $\mathcal{T}$ contains a single $\mathscr{A}$ node, $\widetilde{\mathfrak{A}}_{m}^{T}$ is $\widetilde{\mathfrak{A}}_{m, 1}^{\forall} ; \widetilde{\mathfrak{B}}_{m}^{T}$ is $\widetilde{\mathfrak{B}}_{m, 1}^{\forall}$.

- When $|r k(\mathcal{T})|>1$ :

Assume that the root $r$ of $\mathcal{T}$ has $k$ children, and the maximal subtrees that are rooted at these children are $\mathcal{T}_{1}, \cdots, \mathcal{T}_{k}$ respectively. Recall that $\mathcal{T}$ is an irreducible tree, which implies $\mathcal{T}_{i}$ and $\mathcal{T}_{j}$ are not isomorphic if $i \neq j$.

For any $\Gamma$-labelled tree $\mathcal{T}^{\prime}$, let $\widetilde{\mathfrak{A}}_{m}^{-T^{\prime}}$ be the same as $\widetilde{\mathfrak{A}}_{m}^{T^{\prime}}$ except that the colours of all the edges are exchanged, i.e. red is exchanged with blue. Let $P_{i}$, which is a member of the set $\{(A, A),(A, B),(B, A),(B, B), A, B\}$, and $\mathfrak{D}_{T_{1}, \cdots, T_{k}, m}^{P_{1}, \cdots, P_{k}}$, which is a $\left(\tau^{+} \cup\{\mathfrak{e}\} \backslash\{r\}\right)$-structure where $\mathfrak{e}$ is a hook constant, be built by the following process:

step 1: Let $M:=\emptyset$;

step 2: For $i=1$ to $k$ do the following:

- if $P_{i}=(A, A)$ then $M:=M \cup\left\{\widetilde{\mathfrak{A}}_{m}^{T_{i}}, \widetilde{\mathfrak{A}}_{m}^{-T_{i}}\right\} ;$

- if $P_{i}=(A, B)$ then $M:=M \cup\left\{\widetilde{\mathfrak{A}}_{m}^{T_{i}}, \widetilde{\mathfrak{B}}_{m}^{-T_{i}}\right\}$;

- if $P_{i}=(B, A)$ then $M:=M \cup\left\{\widetilde{\mathfrak{B}}_{m}^{T_{i}}, \widetilde{\mathfrak{A}}_{m}^{-T_{i}}\right\}$;

- if $P_{i}=(B, B)$ then $M:=M \cup\left\{\widetilde{\mathfrak{B}}_{m}^{T_{i}}, \widetilde{\mathfrak{B}}_{m}^{-T_{i}}\right\}$;

- if $P_{i}=A$ then $M:=M \cup\left\{\widetilde{\mathfrak{A}}_{m}^{T_{i}}\right\}$;

- if $P_{i}=B$ then $M:=M \cup\left\{\widetilde{\mathfrak{B}}_{m}^{T_{i}}\right\}$;

step 3: join all the trees in $M$ at their roots. Call their shared root a junction point, which interprets the hook constant $\mathfrak{e}$.

We use $O_{i}$ to denote the label of the root of the tree $\mathcal{T}_{i}$. Let $H:=\left\{i \in[1, k] \mid\right.$ the root of $\mathcal{T}_{i}$ has the same label as that of the root of $\mathcal{T}$ \}.

In the following, we define some substructure for the constructions:

- For any $j \in H$, let $\mathfrak{C}_{\exists, j, m}^{A, B, T}:=\mathfrak{D}_{T_{1}, \cdots, T_{k}, m}^{P_{1}, \cdots, P_{k}}$ where $P_{j}=(A, B)$ and $[$ if $i \neq j$ then $\left(P_{i}=(A, A)\right.$ if $\left.O_{i}=\exists\right)$ and $\left(P_{i}=A\right.$ if $\left.\left.O_{i}=\forall\right)\right]$;

- For any $j \in H$, let $\mathfrak{C}_{\exists, j, m}^{B, A, T}:=\mathfrak{D}_{T_{1}, \cdots, T_{k}, m}^{P_{1}, \cdots, P_{k}}$ where $P_{j}=(B, A)$ and [ if $i \neq j$ then $\left(P_{i}=(A, A)\right.$ if $\left.O_{i}=\exists\right)$ and $\left(P_{i}=A\right.$ if $\left.\left.O_{i}=\forall\right)\right]$;

- For any $j \in[1, k]$ and $j \notin H$, let $\mathfrak{C}_{\exists, j, m}^{B, B, T}:=\mathfrak{D}_{T_{1}, \cdots, T_{k}, m}^{P_{1}, \cdots, P_{k}}$ where $P_{j}=B$ and $[$ if $i \neq$ $j$ then $\left(P_{i}=(A, A)\right.$ if $\left.O_{i}=\exists\right)$ and $\left(P_{i}=A\right.$ if $\left.\left.O_{i}=\forall\right)\right]$;

- We use $\mathfrak{C}_{\exists, 0, m}^{A, A, T}$ to denote the structure $\mathfrak{D}_{T_{1}, \cdots, T_{k}, m}^{P_{1}, \cdots, P_{k}}$ where $P_{i}=(A, A)$ if $O_{i}=\exists ; P_{i}=A$ if $O_{i}=\forall$.

Dually, we can define $\mathfrak{C}_{\forall, j, m}^{B, A}, \mathfrak{C}_{\forall, j, m}^{A, B, T}, \mathfrak{C}_{\forall, j, m}^{A, A}, \mathfrak{C}_{\forall, 0, m}^{B, B, T}$ as follows:

- For any $j \in H$, let $\mathfrak{C}_{\forall, j, m}^{A, B, T}:=\mathfrak{D}_{T_{1}, \cdots, T_{k}, m}^{P_{1}, \cdots, P_{k}}$ where $P_{j}=(A, B)$ and [ if $i \neq j$ then $\left(P_{i}=(B, B)\right.$ if $\left.O_{i}=\forall\right)$ and $\left(P_{i}=B\right.$ if $\left.\left.O_{i}=\exists\right)\right]$;

- For any $j \in H$, let $\mathfrak{C}_{\forall, j, m}^{B, A, T}:=\mathfrak{D}_{T_{1}, \cdots, T_{k}, m}^{P_{1}, \cdots, P_{k}}$ where $P_{j}=(B, A)$ and $[$ if $i \neq j$ then $\left(P_{i}=(B, B)\right.$ if $\left.O_{i}=\forall\right)$ and $\left(P_{i}=B\right.$ if $\left.\left.O_{i}=\exists\right)\right]$;

- For $j \notin H$, let $\mathfrak{C}_{\forall, j, m}^{A, A, T}:=\mathfrak{D}_{T_{1}, \cdots, T_{k}, m}^{P_{1}, \cdots, P_{k}}$ where $P_{j}=A$ and $\left[\right.$ if $i \neq j$ then $\left(P_{i}=(B, B)\right.$ if $\left.O_{i}=\forall\right)$ and $\left(P_{i}=B\right.$ if $\left.\left.O_{i}=\exists\right)\right]$;

- We use $\mathfrak{C}_{\forall, 0, m}^{B, B, T}$ to denote the structure $\mathfrak{D}_{T_{1}, \cdots, T_{k}, m}^{P_{1}, \cdots, P_{k}}$ where $P_{i}=(B, B)$ if $O_{i}=\forall ; P_{i}=B$ if $O_{i}=\exists$.

As usual, we define the main structures based on point-expansions over some sets of structures. Now we define such sets.

$-\mathcal{K}_{0}^{\exists, T}:=\left\{\mathfrak{C}_{\exists, i, m}^{A, B, T} \mid i \in H\right\} \cup\left\{\mathfrak{C}_{\exists, i, m}^{B, A, T} \mid i \in H\right\} \cup\left\{\mathfrak{C}_{\exists, i, m}^{B, B, T} \mid i \in[1, k]\right.$ and $\left.i \notin H\right\} ;$ 
$-\mathcal{K}_{A}^{\exists, T}:=\left\{\mathfrak{C}_{\exists, 0, m}^{A, A, T}\right\} \cup\{\mathcal{I}\} \cup \mathcal{K}_{0}^{\exists, T}$

$-\mathcal{K}_{B}^{\exists, T}:=\{\mathcal{I}\} \cup \mathcal{K}_{0}^{\exists, T}$.

$-\mathcal{K}_{0}^{\forall, T}:=\left\{\mathfrak{C}_{\forall, i, m}^{A, B, T} \mid i \in H\right\} \cup\left\{\mathfrak{C}_{\forall, i, m}^{B, A, T} \mid i \in H\right\} \cup\left\{\mathfrak{C}_{\forall, i, m}^{A, A, T} \mid i \in[1, k]\right.$ and $\left.i \notin H\right\} ;$

$-\mathcal{K}_{A}^{\forall, T}:=\{\mathcal{I}\} \cup \mathcal{K}_{0}^{\forall, T}$

$-\mathcal{K}_{B}^{\forall, T}:=\left\{\mathfrak{C}_{\forall, 0, m}^{B, B, T}\right\} \cup\{\mathcal{I}\} \cup \mathcal{K}_{0}^{\forall, T}$.

Now we define $\widetilde{\mathfrak{A}}_{m}^{T}$ and $\widetilde{\mathfrak{B}}_{m}^{T}$ as follows:

- If the root of $\mathcal{T}$ is an $\mathscr{E}$ node, which is connected to the roots of $k$ trees $\mathcal{T}_{1}, \cdots, \mathcal{T}_{k}$, and assume that the number of $\exists$ in the tuple $\left(O_{1}, \cdots, O_{k}\right)$ is $\jmath$, then $\widetilde{\mathfrak{A}}_{m}^{T}$ and $\widetilde{\mathfrak{B}}_{m}^{T}$ are defined as follows:

* $\widetilde{\mathfrak{A}}_{m}^{T}$ is a point-expansion of $\mathcal{T}_{m, \jmath+k}^{\mathfrak{A}, \exists}$ over $\mathcal{K}_{A}^{\exists, T}$ : The root of $\mathcal{T}_{m, j+k}^{\mathfrak{A}, \exists}$ is expanded by $\mathcal{I}$. It is also called the "root" of $\widetilde{\mathfrak{A}}_{m}^{T}$ that interprets $r$. One leaf is expanded by a copy of $\mathfrak{C}_{\exists, 0, m}^{A, A, T}$. For each element of $\mathcal{K}_{0}^{\exists, T}$ there are exactly $m$ distinct leaves which are expanded by it.

* $\widetilde{\mathfrak{B}}_{m}^{T}$ is a point-expansion of $\mathcal{T}_{m, \jmath+k}^{\mathfrak{B}, \exists}$ over $\mathcal{K}_{B}^{\exists, T}$, similar to $\widetilde{\mathfrak{A}}_{m}^{T}$ : The root of $\mathcal{T}_{m, \jmath+k}^{\mathfrak{B}, \exists}$ is expanded by $\mathcal{I}$. For each element of $\mathcal{K}_{0}^{\exists, T}$ there are exactly $m$ distinct leaves which are expanded by it.

- If the root of $\mathcal{T}$ is an $\mathscr{A}$ node, which is connected to the roots of $k$ trees $\mathcal{T}_{1}, \cdots, \mathcal{T}_{k}$, and assume that the number of $\forall$ in the tuple $\left(O_{1}, \cdots, O_{k}\right)$ is $\jmath$, then $\widetilde{\mathfrak{A}}_{m}^{T}$ and $\widetilde{\mathfrak{B}}_{m}^{T}$ are defined as follows:

* $\widetilde{\mathfrak{A}}_{m}^{T}$ is a point-expansion of $\mathcal{T}_{m, \jmath+k}^{\mathfrak{A}, \forall}$ over $\mathcal{K}_{A}^{\forall, T}$ : The root of $\mathcal{T}_{m, \jmath+k}^{\mathfrak{A}, \forall}$ is expanded by $\mathcal{I}$. For each element of $\mathcal{K}_{0}^{\forall, T}$ there are exactly $m$ distinct leaves which are expanded by it.

* $\widetilde{\mathfrak{B}}_{m}^{T}$ is a point-expansion of $\mathcal{T}_{m, \jmath+k}^{\mathfrak{B}, \forall}$ over $\mathcal{K}_{B}^{\forall, T}$ : The root of $\mathcal{T}_{m, \forall+k}^{\mathfrak{B}, \forall}$ is expanded by $\mathcal{I}$. One leaf is expanded by a copy of $\mathfrak{C}_{\forall, 0, m}^{B, B, T}$. For each element of $\mathcal{K}_{0}^{\forall, T}$ there are exactly $m$ distinct leaves which are expanded by it.

Example 5.4. Let $\mathcal{T}$ be a $\Gamma$-labelled irreducible binary tree. Assume that its root is an $\mathscr{E}$ node and is connected to two subtrees $\mathcal{T}_{1}$ and $\mathcal{T}_{2}$, where the root of $\mathcal{T}_{1}$ is an $\mathscr{E}$ node and the root of $\mathcal{T}_{2}$ is an $\mathscr{A}$ node. See Figure 8 for the illustration of the structures $\widetilde{\mathfrak{A}}_{m}^{T}$ and $\widetilde{\mathfrak{B}}_{m}^{T}$. A "**" at the root of a subtree $\mathcal{T}^{\prime}$ means that we have $m$ disjoint isomorphic copies of this tree $\mathcal{T}^{\prime}$ and for each copy we add an edge between the root, $r$, of the whole structure and the root of this copy.

Now we define a sentence $\widetilde{\phi}_{\mathcal{T}}$ over the signature $\tau^{+}$such that, for any $m, \widetilde{\mathfrak{A}}_{m}^{T} \models \widetilde{\phi}_{\mathcal{T}}$ but $\widetilde{\mathfrak{B}}_{m}^{T} \not \widetilde{\phi}_{\mathcal{T}}$.

Definition 5.5. Let $\mathcal{T}$ be a $\Gamma$-labelled irreducible $k$-ary tree, which is connected to the roots of $k$ trees $\mathcal{T}_{1}, \cdots, \mathcal{T}_{k}$. Assume that $r k(\mathcal{T})=d$. Recall that $H=\left\{i \in[k] \mid\right.$ the root of $\mathcal{T}_{i}$ has the same label as that of the root of $\mathcal{T}\}$. We define $\tau^{+}$-sentences $\widetilde{\phi}_{\mathcal{T}}$ and $\widetilde{\phi}_{-} \mathcal{T}$ based on the tree $\mathcal{T}$ as follows:

(1) If $\mathcal{T}$ is empty, then

$$
\widetilde{\xi}_{\mathcal{T}}(x)=\widetilde{\xi}_{-\mathcal{T}}(x):=U(x)
$$

(2) If the root of $\mathcal{T}$ is an $\mathscr{E}$ node, then

$$
\widetilde{\xi}_{\mathcal{T}}(y):=\exists x_{d}\left(R y x_{d} \wedge \bigwedge_{i \in[k]} \widetilde{\xi}_{\mathcal{T}_{i}}\left(x_{d}\right) \wedge \bigwedge_{j \in H} \widetilde{\xi}_{-\mathcal{T}_{j}}\left(x_{d}\right)\right)
$$



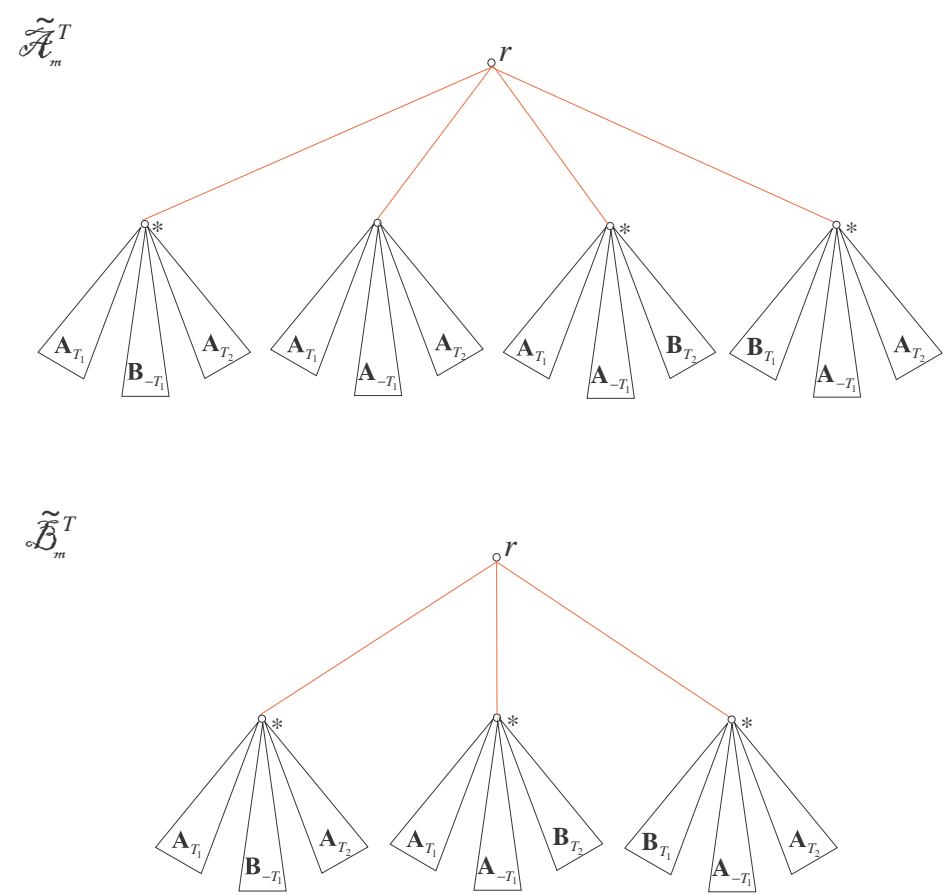

Figure 8: The structures $\widetilde{\mathfrak{A}}_{m}^{T}$ and $\widetilde{\mathfrak{B}}_{m}^{T}$, where $\mathcal{T}$ is an irreducible binary tree, which is connected to $\mathcal{T}_{1}$ and $\mathcal{T}_{2}$. The root of $\mathcal{T}$ is an $\mathscr{E}$ node; The root of $\mathcal{T}_{1}$ is an $\mathscr{E}$ node; and the root of $\mathcal{T}_{2}$ is an $\mathscr{A}$ node. In the figure, $A_{T_{i}}$ and $B_{T_{i}}$ represent $\widetilde{\mathfrak{A}}_{m}^{T_{i}}$ and $\widetilde{\mathfrak{B}}_{m}^{T_{i}}$ respectively.

$$
\widetilde{\xi}_{-\mathcal{T}}(y):=\exists x_{d}\left(B y x_{d} \wedge \bigwedge_{i \in[k]} \widetilde{\xi}_{-\mathcal{T}_{i}}\left(x_{d}\right) \wedge \bigwedge_{j \in H} \widetilde{\xi}_{\mathcal{T}_{j}}\left(x_{d}\right)\right)
$$

(3) If the root of $\mathcal{T}$ is an $\mathscr{A}$ node, then

$$
\begin{aligned}
\widetilde{\xi}_{\mathcal{T}}(y) & :=\forall x_{d}\left(R y x_{d} \rightarrow \bigvee_{i \in[k]} \widetilde{\xi}_{\mathcal{T}_{i}}\left(x_{d}\right) \vee \bigvee_{j \in H} \widetilde{\xi}_{-\mathcal{T}_{j}}\left(x_{d}\right)\right) ; \\
\widetilde{\xi}_{-\mathcal{T}}(y) & :=\forall x_{d}\left(B y x_{d} \rightarrow \bigvee_{i \in[k]} \widetilde{\xi}_{-\mathcal{T}_{i}}\left(x_{d}\right) \vee \bigvee_{j \in H} \widetilde{\xi}_{\mathcal{T}_{j}}\left(x_{d}\right)\right) ;
\end{aligned}
$$

Now, $\widetilde{\phi}_{\mathcal{T}}$ and $\widetilde{\phi}_{-\mathcal{T}}$ are defined as:

$$
\begin{aligned}
\widetilde{\phi}_{\mathcal{T}} & :=\widetilde{\xi}_{\mathcal{T}}(r) ; \\
\widetilde{\phi}_{-\mathcal{T}} & :=\widetilde{\xi}_{-\mathcal{T}}(r) .
\end{aligned}
$$


5.2. The duplicator's winning strategy. In the following, we show that a refined strict quantifier hierarchy exists by proving that the duplicator has a winning strategy in the games that we introduced before. Since the proof resembles that in Section 3, we sketch the main ideas and omit similar details.

Lemma 5.6. Let $S_{1}$ and $S_{2}$ be two finite $\Gamma$-labelled forests. If $S_{1} \npreceq_{e} S_{2}$, then there is an irreducible subtree $\mathcal{T}$ in $S_{1}$ such that the duplicator has a winning strategy in $G_{S_{2}}\left(\widetilde{\mathfrak{A}}_{m}^{T}, \widetilde{\mathfrak{B}}_{m}^{T}\right)$ for any $m \geq r k\left(S_{2}\right)$.

Proof. Observe that when $r k\left(S_{1}\right)=1$, i.e. the rank of $S_{1}$ is $1, S_{1} \npreceq e S_{2}$ implies $\mathscr{W}\left(S_{1}\right) \nsubseteq$ $\mathscr{W}\left(S_{2}\right)$. According to Lemma 3.22 , the duplicator has a winning strategy.

Assume that the lemma holds when $r k\left(S_{1}\right) \leq h$.

Now assume that $r k\left(S_{1}\right)=h+1$.

Because $S_{1} \npreceq_{e} S_{2}$, for some $k \in \mathbb{N}^{+}$there exists a $k$-ary subtree $\mathcal{T}$ in $S_{1}$ such that $\mathcal{T} \npreceq_{e} \mathcal{T}^{\prime}$ for any subtree $\mathcal{T}^{\prime}$ in $S_{2}$. Moreover, we assume that $\mathcal{T}$ is the minimal subtree in $S_{1}$ that is not embeddable in $S_{2}$, i.e. any subtree of $\mathcal{T}$, which is not $\mathcal{T}$ itself, is embeddable in $S_{2}$. Note that such a tree is an irreducible tree, whose rank is no larger than $h+1$. Here we use $r(\mathcal{T})$ to denote the root of $\mathcal{T}$. And we assume that $r(\mathcal{T})$ is an $\mathscr{E}$ node. We assume that there are $1 \leq k^{\prime} \leq k$ subtrees, $\mathcal{T}_{1}, \cdots, \mathcal{T}_{k^{\prime}}$, whose roots are connected to $r(\mathcal{T})$. Let $\mathrm{Mg}$ be the set of $\mathscr{E}$ nodes in $S_{2}$ such that for any $a \in \mathrm{Mg}$ no other $\mathscr{E}$ node appears in the path from the root of $S_{2}$ to $a$. By assumption $\mathcal{T}$ is not embeddable in any subtree of $S_{2}$. For any $a \in \mathrm{Mg}$, let $\mathcal{T}^{\prime}$ be a tree rooted at $a$ and $\mathcal{F}$ be the forest obtained from $\mathcal{T}^{\prime}$ by removing the root of $\mathcal{T}^{\prime}$. Hence, at least one of the trees $\mathcal{T}_{1}, \cdots, \mathcal{T}_{k^{\prime}}$ cannot be embedded in $\mathcal{F}$. Observe that $r k\left(\mathcal{T}_{i}\right) \leq h$ for any $i \in\left[k^{\prime}\right]$.

Note that, in any play of the game, the moving track of the token in $S_{2}$ is a directed path. If the first place where the token lies is an $\mathscr{A}$ node, then the path is initiated with a block of universal quantifiers. However, in the rounds based on this first block of universal quantifiers the spoiler has to pick in $\widetilde{\mathfrak{B}}_{m}^{T}$ and no matter how he picks the duplicator can mimic his picking in the isomorphic subtrees. By Lemma 3.20, if the spoiler can win the game by picking these elements, he can also win the game without picking these elements.

When the token is on a node of $\mathrm{Mg}$, say $a$, we assume that the spoiler picks in $\mathfrak{C}_{\exists, 0, m}^{A, A}$ (i.e. in a copy of $\widetilde{\mathfrak{A}}_{m}^{T}$ ) because if he picks in other places the duplicator can mimic his picking in an isomorphic tree substructure, hence by Lemma 3.20 the spoiler can win the game without picking in these places if he has at least one winning strategy. In this round, the duplicator's strategy is as follows (i.e. picking in a copy of $\widetilde{\mathfrak{B}}_{m}^{T}$ ):

- If $\mathcal{T}_{i}$ is not embeddable in the forest $\mathcal{F}$ and $r\left(\mathcal{T}_{i}\right)$ is an $\mathscr{E}$ node, then

- if the spoiler picks inside $\widetilde{\mathfrak{A}}_{m}^{T_{i}}$, which is a part of $\mathfrak{C}_{\exists, 0, m}^{A, A, T}$, then the duplicator mimics it in an isomorphic copy of $\widetilde{\mathfrak{A}}_{m}^{T_{i}}$, which is a part of $\mathfrak{C}_{\exists, i, m}^{A, B, T}$.

- if the spoiler picks inside $\widetilde{\mathfrak{A}}_{m}^{-T_{i}}$, which is a part of $\mathfrak{C}_{\exists, 0, m}^{A, A, T}$, then the duplicator mimics it in an isomorphic copy of $\widetilde{\mathfrak{A}}_{m}^{-T_{i}}$, which is a part of $\mathfrak{C}_{\exists, i, m}^{B, A, T}$.

- if the spoiler picks inside $\widetilde{\mathfrak{A}}_{m}^{T_{j}}$ or $\widetilde{\mathfrak{A}}_{m}^{-T_{j}}$ where $j \neq i$, then the duplicator mimics it in an isomorphic copy of $\widetilde{\mathfrak{A}}_{m}^{T_{j}}$ or $\widetilde{\mathfrak{A}}_{m}^{-T_{j}}$, which is a part of $\mathfrak{C}_{\exists, i, m}^{A, B, T}$.

- if the spoiler picks the junction point, i.e. the root, of $\mathfrak{C}_{\exists, 0, m}^{A, A, T}$, then the duplicator picks the junction point of $\mathfrak{C}_{\exists, i, m}^{A, B, T}$.

- If $\mathcal{T}_{i}$ is not embeddable in $\mathcal{F}$ and $r\left(\mathcal{T}_{i}\right)$ is an $\mathscr{A}$ node, then 
- if the spoiler picks inside $\widetilde{\mathfrak{A}}_{m}^{T_{i}}$, which is a part of $\mathfrak{C}_{\exists, 0, m}^{A, A, T}$, the duplicator is able to mimic the spoiler's picks inside $\widetilde{\mathfrak{B}}_{m}^{T_{i}}$, which is a part of $\mathfrak{C}_{\exists, i, m}^{B, B, T}$. It is because the root of $\mathcal{T}_{i}$ is an $\mathscr{A}$ node, which means that the number of different types of subtrees, whose roots are connected to the root of $\widetilde{\mathfrak{A}}_{m}^{T_{i}}$ (in $\widetilde{\mathfrak{A}}_{m}^{T}$ ), is less than the number of different types of subtrees, whose roots are connected to the root of $\widetilde{\mathfrak{B}}_{m}^{T_{i}}$ (in $\widetilde{\mathfrak{B}}_{m}^{T}$ ).

- if the spoiler picks inside $\widetilde{\mathfrak{A}}_{m}^{T_{j}}$ or $\widetilde{\mathfrak{A}}_{m}^{-T_{j}}$ where $j \neq i$, then the duplicator mimics it in an isomorphic copy of $\widetilde{\mathfrak{A}}_{m}^{T_{j}}$ or $\widetilde{\mathfrak{A}}_{m}^{-T_{j}}$, which is a part of $\mathfrak{C}_{\exists, i, m}^{B, B, T}$.

- if the spoiler picks the junction point, i.e. the root, of $\mathfrak{C}_{\exists, 0, m}^{A, A, T}$, then the duplicator picks the junction point of $\mathfrak{C}_{\exists, i, m}^{B, B, T}$.

Note that such a strategy has exploited the feature of the structures on the game board: (recall that the root of $\mathcal{T}$ is an $\mathscr{E}$ node) the structures are constructed in such a way that if the spoiler does not pick the junction point of $\mathfrak{C}_{\exists, 0, m}^{A, A, T}$ in $\widetilde{\mathfrak{A}}_{m}^{T}$ then the duplicator is able to mimic his picking in an isomorphic tree substructure of $\widetilde{\mathfrak{B}}_{m}^{T}$ - such isomorphic subtrees always exist. And by Lemma 3.20 the spoiler can also win without such picking if he can win in any way. If the spoiler pick the junction point of $\mathfrak{C}_{\exists, 0, m}^{A, A, T}$, by her strategy, the duplicator picks the junction point of $\mathfrak{C}_{\exists, i, m}^{A, B, T}$ where $\mathcal{T}_{i} \npreceq_{e} \mathcal{F}$. Observe that, the difference between $\mathfrak{C}_{\exists, 0, m}^{A, A, T}$ and $\mathfrak{C}_{\exists, i, m}^{A, B, T}$ is the difference between $\widetilde{\mathfrak{A}}_{m}^{-T_{i}}$ and $\widetilde{\mathfrak{B}}_{m}^{-T_{i}}$ (or equivalently the difference between $\widetilde{\mathfrak{A}}_{m}^{T_{i}}$ and $\widetilde{\mathfrak{B}}_{m}^{T_{i}}$ ). Therefore, using this strategy, no matter how the spoiler picks, the duplicator can reply properly such that in the end the spoiler can win the game $G_{\mathcal{F}}\left(\widetilde{\mathfrak{A}}_{m}^{-T_{i}}, \widetilde{\mathfrak{B}}_{m}^{-T_{i}}\right.$ ) (or $\left.G_{\mathcal{F}}\left(\widetilde{\mathfrak{A}}_{m}^{T_{i}}, \widetilde{\mathfrak{B}}_{m}^{T_{i}}\right)\right)$, if the spoiler can win in any way. Recall that $r k\left(\mathcal{T}_{i}\right) \leq h$. By induction assumption, the duplicator can win the game using this strategy. Therefore, the spoiler cannot win the game if the duplicator plays according to this strategy. In other words, this strategy is a winning strategy for the duplicator in the game $G_{S_{2}}\left(\widetilde{\mathfrak{A}}_{m}^{T}, \widetilde{\mathfrak{B}}_{m}^{T}\right)$.

When $r(\mathcal{T})$ is an $\mathscr{A}$ node, the analysis is similar.

Note that the duplicator's winning strategy in Lemma 5.6 depends on how the spoiler moves the token. That is, she has to keep an eye on the token track before she make the choices.

The following corollary is directly due to Lemma 3.6 and Theorem 3.8 .

Corollary 5.7. Let $S_{1}$ and $S_{2}$ be two finite $\Gamma$-labelled forests. If $S_{1} \npreceq e S_{2}$, then for any $\psi$ such that $q s(\psi) \preceq_{e} S_{2}$, and any $m \geq \operatorname{rk}\left(S_{2}\right)$, we have:

$$
\widetilde{\mathfrak{A}}_{m}^{S_{1}}=\psi \Rightarrow \widetilde{\mathfrak{B}}_{m}^{S_{1}} \models \psi .
$$

The following lemma is similar to Lemma 3.17. Actually, the proof resembles that of Lemma 3.17.

Lemma 5.8. Let $\mathcal{T}$ be $\Gamma$-labelled irreducible tree. Then

$$
\widetilde{\mathfrak{A}}_{m}^{T}=\widetilde{\phi}_{\mathcal{T}} \text { but } \widetilde{\mathfrak{B}}_{m}^{T} \not \models \widetilde{\phi}_{\mathcal{T}} \text {. }
$$

Proof. It is obvious when $\mathcal{T}$ is a single node. Assume that it holds when $r k(\mathcal{T})=h$.

Now assume that $\mathcal{T}$ is a $k$-ary $\Gamma$-labelled tree and its rank is $h+1$. Suppose that the root of $\mathcal{T}$ is an $\mathscr{E}$ node, and its subtrees, whose roots are children of $r(\mathcal{T})$, are $\mathcal{T}_{1}, \cdots, \mathcal{T}_{k}$. Recall that $H=\left\{i \in[k] \mid\right.$ the root of $\mathcal{T}_{i}$ has the same label as that of the root of $\left.\mathcal{T}\right\}$. 
Let $a_{r}$ be the node that interprets $r$ in $\widetilde{\mathfrak{A}}_{m}^{T}$. And let $b_{r}$ be the node that interprets the hook constant in $\mathfrak{C}_{\exists, 0, m}^{A, A, T}$, i.e. $b_{r}$ is the junction point of $\mathfrak{C}_{\exists, 0, m}^{A, A, T}$. By assumption and an observation similar to Lemma 3.16, $\widetilde{\mathcal{A}}_{m}^{T_{i}}=\widetilde{\xi}_{\mathcal{T}_{i}}(r)(i \in[k])$, and $\widetilde{\mathcal{A}}_{m}^{-T_{j}}=\widetilde{\xi}_{-\mathcal{T}_{j}}(r)(j \in H)$.

Moreover, all the quantifiers in $\widetilde{\xi}_{\mathcal{T}_{i}}(r)$ are relativized by relations either $R y x$ or $B y x$, where $x$ is the quantified variable. It means that $\widetilde{\xi}_{\mathcal{T}_{i}}(r)$ expresses some property that is nothing to do with the elements of $\widetilde{\mathfrak{A}}_{m}^{T}$ outside the tree substructure $\widetilde{\mathfrak{A}}_{m}^{T_{i}}$. As a consequence, $\widetilde{\mathfrak{A}}_{m}^{T_{i}} \models \widetilde{\xi}_{\mathcal{T}_{i}}(r)$ implies $\widetilde{\mathfrak{A}}_{m}^{T} \models \widetilde{\xi}_{\mathcal{T}_{i}}\left(b_{r}\right)$.

By the same argument, $\widetilde{\mathfrak{A}}_{m}^{-T_{i}} \models \widetilde{\xi}_{-\mathcal{T}_{i}}(r)$ implies $\widetilde{\mathfrak{A}}_{m}^{T} \models \widetilde{\xi}_{-\mathcal{T}_{i}}\left(b_{r}\right)$.

Therefore, in $\widetilde{\mathfrak{A}}_{m}^{T}, b_{r}$ is the witness of the quantifier $\exists x_{d}$ in the formula $\widetilde{\xi}_{\mathcal{T}}(r)$. That is, $\widetilde{\mathfrak{A}}_{m}^{T}=\widetilde{\xi}_{\mathcal{T}}(r)$, or $\widetilde{\mathfrak{A}}_{m}^{T}=\widetilde{\phi}_{\mathcal{T}}$.

Likewise, by assumption and similar analysis, in $\widetilde{\mathfrak{B}}_{m}^{T}$ the subtrees $\mathfrak{C}_{\exists, i, m}^{A, B, T}$, or $\mathfrak{C}_{\exists, i, m}^{B, A, T}$, or $\mathfrak{C}_{\exists, i, m}^{B, B, T}$, has exactly one subtree $\widetilde{\mathfrak{B}}_{m}^{T_{i}}$, or $\widetilde{\mathfrak{B}}_{m}^{-T_{i}}$, which does not satisfy some subformula of $\widetilde{\phi}_{\mathcal{T}}$ (i.e. one conjunct of $\bigwedge_{i \in[} \widetilde{\xi}_{\mathcal{T}_{i}}\left(x_{d}\right) \wedge \bigwedge_{j \in H} \widetilde{\xi}_{-\mathcal{T}_{j}}\left(x_{d}\right)$ ), when its root interprets $x_{d}$. In other $i \in[k] \quad j \in H$

words, $b_{r}$ cannot be a witness of the quantifer $\exists x_{d}$. Therefore, $\widetilde{\mathfrak{B}}_{m}^{T} \not \widetilde{\phi}_{\mathcal{T}}$.

When the root of $\mathcal{T}$ is an $\mathscr{A}$ node, the analysis is similar.

Theorem 5.9. Let $S_{1}$ and $S_{2}$ be two $\Gamma$-labelled forests. Over the class of all finite $\tau^{+}$. structures,

$$
\text { if } S_{1} \npreceq e S_{2} \text {, then } \mathrm{FO}\left\{S_{1}\right\} \nsubseteq \mathrm{FO}\left\{S_{2}\right\} \text {. }
$$

Proof. By Lemma 5.6, we know that the duplicator has a winning strategy in the game $G_{S_{2}}\left(\widetilde{\mathfrak{A}}_{m}^{S_{1}}, \widetilde{\mathfrak{B}}_{m}^{S_{1}}\right)$. In other words, the property defined by $\widetilde{\phi}_{\mathcal{T}}$ is not expressible in $\mathrm{FO}\left\{S_{2}\right\}$, by Lemma 3.9. Observe that $\widetilde{\phi}_{\mathcal{T}} \in \mathrm{FO}\left\{S_{1}\right\}$. Therefore, $\operatorname{FO}\left\{S_{1}\right\} \nsubseteq \operatorname{FO}\left\{S_{2}\right\}$.

Recall that $\tau=\langle E\rangle$. Based on the same transformations as the "reductions from $\tau^{+}$to $\tau$ " (p. 20), the diagraph $\mathfrak{A}_{m}^{T}$ ( $\mathfrak{B}_{m}^{T}$ resp.) is obtained from $\widetilde{\mathfrak{A}}_{m}^{T}\left(\widetilde{\mathfrak{B}}_{m}^{T}\right.$ resp.), as $\mathfrak{A}_{m}^{p}$ ( $\mathfrak{B}_{m}^{p}$ resp.) is obtained from $\widetilde{\mathfrak{A}}_{m}^{p}\left(\widetilde{\mathfrak{B}}_{m}^{p}\right.$ resp.); $\phi_{\mathcal{T}}$ is obtained from $\widetilde{\phi}_{\mathcal{T}}$ as $\varphi_{p}$ is obtained from $\widetilde{\varphi}_{p}$. Recall that the reductions are mainly doing three things:

- change red edges to forward edges; change blue edges to backward edges;

- use self-loops to indicate the positions of junction points;

- use bi-directional edges to indicate the positions of black leaves.

We can use the same reductions, prove a new version of Lemma 3.26 as the following:

For any first-order sentence $\zeta$ over $\tau$, there is a first-order sentence $\xi$ over $\tau^{+}$, with the same quantifier structure, such that

(1) $\mathfrak{A}_{m}^{T} \models \zeta$ iff $\widetilde{\mathfrak{A}}_{m}^{T} \models \xi$;

(2) $\mathfrak{B}_{m}^{T}=\zeta$ iff $\widetilde{\mathfrak{B}}_{m}^{T}=\xi$.

And Theorem 5.1 is immediate, by the same argument in the proof of Theorem 3.1 .

The following is a direct corollary of Theorem 5.1 :

Corollary 5.10. Let $S_{1}$ and $S_{2}$ be two $\Gamma$-labelled forests and $\sigma_{g}$ includes a $k$-ary relation symbol where $k \geq 2$. Over the class of all finite $\sigma_{g}$-structures,

$$
\text { if } S_{1} \npreceq_{e} S_{2} \text {, then FO }\left\{S_{1}\right\} \nsubseteq \mathrm{FO}\left\{S_{2}\right\} \text {. }
$$


Proof. We can use the $k$-ary relation $(k \geq 2)$ to encode the binary relations we defined in the structures for Theorem 5.1 and let all the other relations be empty.

\section{Summary}

It is natural to classify fragments of first-order logic based on quantifier structures. So far, most related works are focused on those fragments based on quantifier prefixes, a special kind of quantifier structure, and contribute to our understanding of their different expressiveness. Grädel-McColm's conjecture, which claims the strictness of the first-order prefix hierarchy, generalizes the results of Walkoe [10], Keisler \& Walkoe [3] and Chandra \& Harel [1]. Rosen proved this conjecture by showing that it holds over infinite structures [6] and raised the question of whether it also holds over finite structures. We define games that characterize the quantifier classes, which generalize the standard Ehrenfeucht-Fraïssé games, and prove that a similar and natural hierarchy, i.e. the first-order quantifier structure hierarchy, is strict over finite structures. Although these two hierarchies are similar, they are independent in that none implies the other directly. Nevertheless, our constructions do provide justifications for some special cases of Grädel-McColm's conjecture over finite structures. For example, from the constructions introduced in this paper we can see that there is a property that is expressible in $\mathrm{FO}(\exists \forall \forall)$, but not in $\mathrm{FO}(\forall \forall \forall), \mathrm{FO}(\forall \forall \exists), \mathrm{FO}(\forall \exists \exists)$ and $\mathrm{FO}(\exists \exists \exists)$. But we don't know whether it is expressible in $\mathrm{FO}(\forall \exists \forall)$ or not.

Recall that the mapping $\iota$ between quantifier structures is not necessarily injective when we define the embedding relation $\preceq_{e}$ (Definition 2.7). Now, we change the definition of quantifier structure embedding a little bit, i.e. require $\iota$ to be injective. As a consequence, the quantifier classes are also changed correspondingly. And a finite version of Rosen's main theorem [6] can be stated as the following, based on such change. Recall that we assume that all the structures are finite.

$$
\text { Let } p \in \Gamma^{*} \text { and } S \text { be a } \Gamma \text {-labelled forest, } \mathrm{FO}(p) \nsubseteq \mathrm{FO}\{S\} \text { if } p \notin \mathscr{W}(S) \text {. }
$$

As has been mentioned in the introduction, a proof of this theorem will solve GrädelMcColm's conjecture over finite structures, which is still open at present. Another possible way to resolve this conjecture is to define Ehrenfeucht-Fraïssé style games for the prefix classes. In any cases, we need new techniques and insights.

Let $\mathcal{T}$ be a $\Gamma$-labelled tree and $\mathcal{T}^{i}$ be a forest composed of $i$ disjoint copies of $\mathcal{T}$. Let $\mu_{t}$ be a map from a $\Gamma$-labelled tree to a natural number such that $\mu_{t}(\mathcal{T})$ is the minimum number for $m$ such that $\operatorname{FO}\left\{\mathcal{T}^{m}\right\}=\left.\operatorname{FO}\left\{\mathcal{T}^{m+1}\right\}\right|^{3}$ Then, the following is conceivable, under the new definition of quantifier structure embedding:

Let $\mathcal{T}$ be a $\Gamma$-labelled tree and $S$ be an arbitrary $\Gamma$-labelled forests. Over the class of all finite digraphs, for any $m \leq \mu_{t}(\mathcal{T})$,

$$
\text { if } \mathcal{T}^{m} \npreceq_{e} S \text {, then } \mathrm{FO}\left\{\mathcal{T}^{m}\right\} \nsubseteq \mathrm{FO}\{S\} \text {. }
$$

Note that it generalizes not only Grädel-McColm's conjecture over finite structures but also the finite version of Rosen's main theorem. Furthermore, it is also interesting to study $\mu_{t}(\mathcal{T})$. For example, how fast will it grow w.r.t. the size of $\mathcal{T}$ ?

Another question is whether we can prove similar hierarchies in other logics. One candidate is so called independence-friendly logic (IFL). What makes it interesting is that IFL has the form of first-order logic, while has the expressive power equals existential

\footnotetext{
${ }^{3}$ The existence of such a number $m$ is determined by Lemma 3.3
} 
second-order logic (ESO). It is well-known that ESO captures the complexity class NP over finite structures. Clearly, establishing a natural and strict hierarchy for the NP problems would be very interesting.

\section{ACKNOWLEDGEMENT}

I am grateful to Anuj Dawar for giving me a lot of suggestions and ideas which improve the paper significantly and to Bjarki Holm for useful discussions and proofreading. I am also grateful to the anonymous referees for their valuable comments.

\section{REFERENCES}

[1] A. Chandra and D. Harel. Structure and complexity of relational queries. Journal of Computer and System Science, 25:99-128, 1982.

[2] E. Grädel and G. McColm. Hierarchies in transitive closure logic, stratified datalog and infinitary logic. Annals of Pure and Applied Logic, 77(2):169-199, 1996.

[3] H. Jerome Keisler and JR. Wilbur Walkoe. The diversity of quantifier prefixes. The Journal of Symbolic Logic, 38:79-85, 1973.

[4] L. Libkin. Elements of Finite Model Theory. Springer, 2004.

[5] E. Rosen. Some aspects of model theory and finite structures. Bulletin of Symbolic Logic, 8(3):380-403, 2002.

[6] E. Rosen. On the first-order prefix hierarchy. Notre Dame J. Formal Logic, (online draft, 1998), 46:147$164,2005$.

[7] N. Schweikardt. Arithmetic, first-order logic, and counting quantifiers. ACM Trans. Comput. Logic, 6(3):634-671, 2005.

[8] N. Schweikardt and T. Schwentick, "A note on the expressive power of linear orders," Logical Methods in Computer Science, 7(4), pp.1-13, 2011.

[9] M. Sipser. Borel sets and circuit complexity. In Proceedings of the fifteenth annual ACM symposium on Theory of computing, pages 61-69. ACM, 1983.

[10] W. Walkoe. Finite partially-ordered quantification. Journal of Symbolic Logic, 35:535-555, 1970.

This work is licensed under the Creative Commons Attribution-NoDerivs License. To view a copy of this license, visit http://creativecommons.org/licenses/by-nd/2.0/ or send a letter to Creative Commons, 171 Second St, Suite 300, San Francisco, CA 94105, USA, or letter to Creative Commons, 171 Second St, Suit
Eisenacher Strasse 2, 10777 Berlin, Germany 